\title{
STRUCTURAL DETERMINANTS OF PROTOCADHERIN-15 ELASTICITY AND FUNCTION IN
}

\section{INNER-EAR MECHANOTRANSDUCTION}

Deepanshu Choudhary ${ }^{1 \S}$, Yoshie Narui ${ }^{1 \S}$, Brandon L. Neel ${ }^{1,2}$, Lahiru N. Wimalasena ${ }^{1}$, Carissa F. Klanseck ${ }^{1}$, Pedro De-la-Torre ${ }^{1}$, Conghui Chen ${ }^{1}$, Raul Araya-Secchi ${ }^{1,3}$, Elakkiya Tamilselvan ${ }^{1,4}$, and Marcos Sotomayor ${ }^{1,2,4} *$

${ }^{1}$ Department of Chemistry and Biochemistry, The Ohio State University $484 \mathrm{~W} 12^{\text {th }}$ Avenue, Columbus, OH 43210

${ }^{2}$ The Ohio State Biochemistry Program, The Ohio State University $484 \mathrm{~W} 12^{\text {th }}$ Avenue, Columbus, OH 43210

${ }^{3}$ Structural Biophysics, Section for Neutron and X-ray Science, Niels Bohr Institute, University of Copenhagen, 2100 Copenhagen, Denmark

${ }^{4}$ Biophysics Program, The Ohio State University $484 \mathrm{~W} 12^{\text {th }}$ Avenue, Columbus, OH 43210

${ }^{\S}$ Co-first authors.

* Corresponding author: sotomayor.8@osu.edu

ORCID: 0000-0002-3333-1805

July 2019 


\section{Abstract (156 Words)}

3 Protocadherin-15 (PCDH15), an atypical member of the cadherin superfamily, is essential for vertebrate hearing

4 and its dysfunction has been associated with deafness and progressive blindness. The PCDH15 ectodomain,

5 made of eleven extracellular cadherin (EC1-11) repeats and a membrane adjacent domain (MAD12), assembles

6 as a parallel homodimer that interacts with cadherin-23 (CDH23) to form the tip link, a fine filament necessary

7 for inner-ear mechanotransduction. Here we report X-ray crystal structures of a PCDH15 + CDH23

8 heterotetrameric complex and ten PCDH15 fragments that were used to build complete high-resolution models

9 of the monomeric PCDH15 ectodomain. Using molecular dynamics (MD) simulations and validated crystal

10 contacts we propose models for complete PCDH15 parallel homodimers and the tip-link bond. Steered MD

11 simulations of these models predict their strength and suggest conditions in which a multimodal PCDH15

12 ectodomain can act as a stiff or soft gating spring. These results provide a detailed view of the first molecular

13 steps in inner-ear sensory transduction. 


\section{INTRODUCTION}

2 Inner-ear sensory perception begins when mechanosensitive ion channels ${ }^{1-8}$ are gated by "tip links", fine protein

3 filaments essential for hearing and balance (Fig. 1A) $)^{9-12}$. Tip links are 150 to $180 \mathrm{~nm}$ long ${ }^{13-16}$, their integrity is

4 calcium $\left(\mathrm{Ca}^{2+}\right)$-dependent ${ }^{10,17}$, and they are made of protocadherin-15 (PCDH15) and cadherin-23 $(\mathrm{CDH} 23)^{18-21}$,

5 two enormous proteins involved in hereditary deafness ${ }^{22,23}$, progressive blindness ${ }^{24}$, and cancer ${ }^{25-29}$. Mature tip

6 links are thought to be heterotetramers formed by parallel (cis) homodimers of PCDH15 interacting in an

7 antiparallel trans mode (tip-to-tip) with cis homodimers of $\mathrm{CDH} 23^{21}$, whereas immature tip links are likely

8 formed by trans interactions of two PCDH15 molecules ${ }^{30,31}$.

10 Some details of the PCDH15 and CDH23 interaction are well understood: immunogold electron microscopy

11 (EM) suggests that tip-link lengths are mostly consistent with the predicted length of the combined proteins

12 interacting tip-to-tip ${ }^{14,21}$; competitive binding of exogenous PCDH15 and CDH23 tip fragments to endogenous

13 proteins blocks regeneration of tip-links and associated transduction currents during hair-cell development and

14 after tip-link rupture with a $\mathrm{Ca}^{2+}$ chelator $^{32}$; and structures of the complex formed by the tips of PCDH15 and

15 CDH23 engaged in a heterodimeric molecular "handshake" have been obtained and validated in vitro and in

$16 v i v o^{33,34}$. Furthermore, mutations that cause deafness in humans and mice have been shown to or are predicted to

17 break the handshake interaction ${ }^{33-36}$. In addition, studies of the CDH23 and PCDH15 heterodimer show that its

18 strength can be tuned by PCDH15 isoforms that have distinct N-terminal tips ${ }^{37}$. The strength of the heterotetrameric bond can be further diversified when considering combinations of PCDH15 isoforms in parallel. However, little was known about the structure of parallel cis dimers formed by PCDH15 until recently ${ }^{38-40}$. While negative staining transmission EM showed conformationally heterogeneous parallel dimers for tip-link components ${ }^{21}$, most extracellular fragments of PCDH15 (and CDH23) studied had been monomeric in solution ${ }^{38,41-45}$. Recent crystals structures of PCDH15 fragments ${ }^{38-40}$ and a low-resolution EM-based model

24 suggest two points of dimerization ${ }^{38,39}$, yet a detailed atomistic-model of the complete ectodomain is still missing. Also, how PCDH15 engages in trans homodimers is unclear, and the structural details and strength of the PCDH15 + CDH23 heterotetrameric bond are not known. 
1 The oligomerization states of PCDH15 and $\mathrm{CDH} 23$ and the architecture of their heterotetrameric bond are

2 important determinants of tip-link mechanics and inner-ear mechanotransduction ${ }^{16,38}$. Disruption of either the

3 complex between PCDH15 and CDH23 or the oligomerization of PCDH15 impairs transduction ${ }^{33,34,38}$. The

4 inner-ear transduction channel is gated by a soft element called the "gating spring", which is either in series with

5 the tip link or the tip link itself ${ }^{46-48,6,8,49}$. Whether cadherin tip links are elastic or rigid has been

6 controversial ${ }^{11,16,42}$. The ultrastructure of tip links suggested a stiff elastic element ${ }^{11}$, but the length of tip links

7 vary in situ ${ }^{13,14}$. PCDH15 and CDH23 feature 11 and 27 extracellular cadherin (EC) "repeats" (Fig. 1B),

8 respectively, and initial studies of the structure and simulated dynamics of the CDH23 EC1-2 tip predicted that

9 these canonical repeats and their linker region fully occupied by $\mathrm{Ca}^{2+}$ ions at sites 1,2 , and 3 would be stiff ${ }^{42}$ (a

10 similar linker region is shown in Fig. 1D). However, while the EC repeats along PCDH15 and CDH23 are

11 predicted to share a common fold, they vary in sequence, which can result in structural heterogeneity, as seen for

12 other long cadherins ${ }^{50,51}$. For instance, crystal structures of PCDH15 EC9-10 revealed a bent and L-shaped Ca ${ }^{2+}$

13 free linker region, with simulations evincing that unbending can provide some elasticity to the tip link ${ }^{43}$.

14 Bending and flexibility at this L-shaped EC9-10 linker region are also observed in low-resolution electron cryo-

15 microscopy (cryo-EM) conformations of a cis dimeric PCDH15 fragment encompassing EC8 down to its

16 transmembrane helix ${ }^{39}$. These results highlight the diverse mechanical responses of various tip-link cadherin

17 fragments.

19 Crystal structures and simulations have also shown that other parts of PCDH15 can be flexible. The PCDH15

20 EC3-4 linker is flexible and binds two $\mathrm{Ca}^{2+}$ ions, instead of three. In addition, this atypical linker binds $\mathrm{Ca}^{2+}$ ions

21 with decreased binding affinity ( $45 \mu \mathrm{M}$ for site 3 and $>100 \mu \mathrm{M}$ for site 2$)$ as compared to the canonical $\mathrm{Ca}^{2+}$ -

22 binding linker of CDH23 EC1-2 (5 $\mu \mathrm{M}$ for site 3, $44 \mu \mathrm{M}$ for site 2 , and $71 \mu \mathrm{M}$ for site 1$)^{42,44}$. Occupancy of

$23 \mathrm{Ca}^{2+}$-binding sites at EC linkers will greatly determine the mechanics of tip links and whether unfolding of EC

24 repeats could occur before unbinding of the handshake bond ${ }^{33}$. Interestingly, bulk endolymphatic $\mathrm{Ca}^{2+}$

25 concentration in the cochlea is tightly regulated and varies along its length from $\sim 20 \mu \mathrm{M}$ (base) to $\sim 40 \mu \mathrm{M}$

26 (apex $)^{52,53}$. However, $\mathrm{Ca}^{2+}$ concentration near cochlear tip links could be significantly larger ${ }^{54,55}$, and vestibular

$27 \mathrm{Ca}^{2+}$ concentrations are $>100 \mu \mathrm{M}^{53,56}$. Yet, the structure and the $\mathrm{Ca}^{2+}$-dependent mechanics of entire 
1 heterotetrameric tip links remain undetermined. Further issues that need to be investigated include how much

$2 \mathrm{Ca}^{2+}$ surrounds tip links in vivo ${ }^{55}$, how affinities for $\mathrm{Ca}^{2+}$ vary across EC linkers, and how these affinities change

3 when PCDH15 and CDH23 form complexes and when they are under tension.

5 To better understand the molecular mechanics of tip links and the first steps of inner-ear mechanotransduction,

6 we have determined eleven X-ray crystal structures of PCDH15 fragments (Fig. 1C, Table 1), which show all of

7 PCDH15's EC repeats, all its EC linkers, and its membrane adjacent domain (MAD12 ${ }^{40}$, also referred to as

$8 \mathrm{SEA}^{57}, \mathrm{PICA}^{38}$, or $\mathrm{EL}^{39}$ ). These structures allowed us to assemble a complete model of the monomeric

9 ectodomain of PCDH15, to suggest models of trans and cis PCDH15 homodimers, and to build a model of the

10 heterotetrameric PCDH15-CDH23 bond. In addition, we used steered molecular dynamics $\left(\mathrm{SMD}^{58-61}\right)$

11 simulations to predict the $\mathrm{Ca}^{2+}$-dependent mechanics of these models, which suggest a multimodal (stiff or soft)

12 elastic response for PCDH15 and indicates some conditions in which PCDH15 can provide both the elasticity

13 and elongation typically associated with the hair-cell gating spring.

\section{RESULTS}

16 Sequence analyses of PCDH15 EC repeats and its MAD12 from different species reveal an overall sequence identity of $\sim 46 \pm 9 \%$, with EC1 and MAD12 being the most conserved ( $\sim 59 \%)$ and EC8 being the least $(\sim 32$ \%). In contrast, comparison among EC repeats (including MAD12) in Homo sapiens (hs) PCDH15 reveals low overall sequence identity $(\sim 21 \%)$, suggesting structural variability throughout various parts of the protein's ectodomain within a single species (Figs. S1, S2, and S3; Table S1). Previously published structures of wild-type (WT) Mus musculus $(\mathrm{mm})$ PCDH15 EC1-2 (bound to $m m$ CDH23 EC1-2) ${ }^{33}, \mathrm{~mm}$ PCDH15 EC1-3 ${ }^{38}, h s$ PCDH15 
1 To gain further insights into the function of specific repeats and to build a complete model of PCDH15, we

worked with multiple WT and mutated protein fragments (rationale for mutations are explained throughout the text). Successful crystallization and structure determination (Table 1 and Table S2) was possible for $\mathrm{mm}$

PCDH15 EC1-2 with a biotin acceptor peptide (BAP); $h s$ PCDH15 EC1-3 with mutations p.G16D, p.N369D,

7 (p.I582T variant); $m m$ PCDH15 EC6-7; and $m m$ PCDH15 EC7-8 (p.V875A variant). All these protein

fragments were purified from bacterial inclusion bodies under denaturing conditions and refolded (see Methods and Table S3). In addition, we solved the structure of the mammalian-expressed, and thus glycosylated, $\mathrm{mm}$ PCDH15 EC9-MAD12 fragment. A summary of the protein structures presented is shown in Figure 1A (see also

Table 1). Below, we discuss overall properties of EC repeats (from $\mathrm{N}$ - to $\mathrm{C}$-terminus) as revealed by these structures, as well as models and simulations of the full-length monomeric and dimeric PCDH15 ectodomain in complex with CDH23 EC1-2 or EC1-3.

\section{Structures of PCDH15's ectodomain N-terminal fragments suggest cis and trans interactions}

Individual EC repeat structures and their relative orientation (secondary and tertiary structure features) determine the architecture and shape of entire cadherin ectodomains. While our structures reveal that all PCDH15 repeats

Fig. S1), there are some significant variations in loops, secondary structure, linker regions, relative orientation of the EC repeats, and oligomerization states that are likely important for PCDH15's function in inner-ear mechanotransduction.

The structure of $\mathrm{mm}$ PCDH15 EC1-2BAP (Fig. 1C) is similar to other structures in which the same protein fragment has been crystallized in complex with $m m$ CDH23 EC1-2 ${ }^{33}$, or as part of $m m$ PCDH15 EC1- $3^{38}$ (core RMSD $<1.5 \AA)$. The $m m$ PCDH15 EC1-2BAP fragment is monomeric in solution, and an analysis of the 
1 PCDH15 trans interactions expected for immature tip links ${ }^{31}$. The $m m$ PCDH15 EC1-2BAP linker region is

2 canonical (Fig. 1D) and is fully occupied by $\mathrm{Ca}^{2+}$ ions at sites 1, 2, and 3, as expected for linker regions with the

$3 \mathrm{Ca}^{2+}$-binding motif NTerm-XEX-DXD-D(R/Y)(D/E)-XDX-DXNDN-CTerm ${ }^{64}$. In contrast, the EC2-3 linkers in

4 the structures of the $h s$ PCDH15 EC1-3 G16D/N369D/Q370N and $h s$ PCDH15 EC2-3 WT fragments (both

5 dimeric in solution) have only two bound $\mathrm{Ca}^{2+}$ ions (sites 2 and 3; Fig. 1E and Fig. S4A). A couple of modified

$6 \mathrm{Ca}^{2+}$-binding motifs in which DYE is p.200NYE202, and DXNDN is p.236DGDDL240, would suggest that

7 impaired $\mathrm{Ca}^{2+}$ binding at site 1 is encoded in the sequence, yet a third structure of a designed mutant $h s$ PCDH15

8 EC2-3 p.V250N that disrupts cis homodimerization has three bound $\mathrm{Ca}^{2+}$ ions (Fig. $1 \mathrm{~F}$ and Fig. S4B). While we

9 cannot rule out crystallization conditions, including $\mathrm{MgCl}_{2}$ (Table S2), as the source of this discrepancy in ion

10 occupancy, we do observe the p.E202 side chain now coordinating $\mathrm{Ca}^{2+}$ at sites 1 and 2 (Fig. S4A,B). These

11 structures suggest that oligomerization can directly or indirectly alter $\mathrm{Ca}^{2+}$ binding at the EC2-3 linker, and

12 effect that might be relevant to other cadherin complexes.

14 Interestingly, the crystal structures of $h s$ PCDH15 EC1-3 G16D/N369D/Q370N and $h s$ PCDH15 EC2-3 WT

15 reveal crystallographic contacts with arrangements that are compatible with both trans and cis bonds for

16 PCDH15 (Fig. 2A and Figs. S5A-C and S6A-C). In both cases, the interfaces involve repeats EC2 and EC3. The antiparallel interface observed in $h s$ PCDH15 EC1-3 G16D/N369D/Q370N positions EC2 and EC3 from one subunit opposite to EC3 and EC2 from the other (interface area of $919 \AA^{2}$ with an aperture angle of 154.5 ; Fig. S5A), thus forming an antiparallel trans bond similar to that observed for clustered and $\delta$ protocadherins where EC1 and EC4 also play a role in the binding interface ${ }^{65-69}$. The crystallographic contact observed in the $h s$ PCDH15 EC2-3 WT structure reveals a potential " $\mathrm{X}$ " dimerization interface $\left(\sim 1,052 \AA^{2}\right.$; Fig. 2A,B) where protomers overlap their linker regions with an aperture angle of $58.75^{\circ}$. In this arrangement the EC2-3 contact forms the hinge of a "molecular scissor" with an estimated $K_{\mathrm{D}}<1 \mu \mathrm{M}$ according to analytical ultracentrifugation sedimentation velocity (AUC-SV) experiments (Fig. 2A-C and Fig. S7A). Both interfaces involve the same sides of EC2 and EC3, suggesting that aperture-angle variations due to some relative displacement of the protomers could result in switching between the antiparallel and X-dimer states, with the latter in principle able to mediate 
2 The X-dimer interface we observed in $h s$ PCDH15 EC2-3 WT is similar to that reported by Dionne et al. ${ }^{38}$,

3 where a crystal structure of the glycosylated $\mathrm{mm}$ PCDH15 EC1-3 WT fragment (PDB: 6CV7) shows an X-dimer

4 with an aperture angle of 59.59 (Fig. S8A). Overlapping the glycosylated $\mathrm{mm}$ PCDH15 EC1-3 WT monomer in

5 the antiparallel configuration of our non-glycosylated hs PCDH15 EC1-3 G16D/N369D/Q370N structure

6 reveals potential steric clashes between sugars at glycosylation site p.N180 and the cysteine-stapled loop in EC3

7 (with poor density in our structure), thus suggesting that a complete antiparallel trans state is not feasible unless

8 the involved cysteine loop rearranges (Fig. S5B). It is possible that variations in the angle of the $\mathrm{X}$ interface

9 controlled by glycosylation lead to transitions from antiparallel to X-dimer states. Of note, some mutations that

10 disrupt the X-dimer (such as p.V250N and p.V250D ${ }^{38}$ in EC2-3) are also predicted to disrupt the PCDH15-

11 PCDH15 antiparallel dimer, and a monomeric state was observed when the p.V250N mutation was incorporated

12 into our glycosylated $\mathrm{mm}$ PCDH15 EC1-4 fragment (Table 2 and Fig. S7B). In contrast, the double mutation

13 p.L306N/p.V307N at the EC3-EC3 protomer interface of the X-dimer is not predicted to disrupt the trans

14 antiparallel interface (Fig. S5A), but both $h s$ PCDH15 EC2-3 p.L306N/p.V307N and $h s$ PCDH15 EC1-3

15 p.L306N/p.V307N are monomers in solution (Table 2). Thus, the antiparallel trans PCDH15-PCDH15 interface

16 may use the X-dimer interaction as a required intermediate or might be short lived ${ }^{70}$. While the partially

17 deleterious impact of a similar set of mutations (including p.V250D) on hair-cell mechanotransduction

18 highlights the relevance of EC2-3 contacts for PCDH15 function in vivo ${ }^{38}$, an evaluation of how these mutations

19 alter PCDH15-PCDH15 tip links ${ }^{31}$ is still missing.

Using our structure of $h s$ PCDH15 EC2-3, we asked if its X-dimeric arrangement would be compatible with the trans heterodimeric "handshake" interaction between PCDH15 EC1-2 and CDH23 EC1-233. Alignment of EC2 repeats of PCDH15 from the handshake and X-dimer structures allowed us to build a model of the PCDH15 and CDH23 heterotetramer without steric clashes, suggesting that this is a valid model for the tip-link bond (obtained independently by Dionne et al. using $\mathrm{mm}$ PCDH15 EC1-3 ${ }^{38}$; Fig. S8B). Superposition of the Danio rerio (dr) CDH23 EC1-3 structure $^{45}$ onto this complex is also compatible with the heterotetrameric bond conformation in 
$1 \mathrm{CDH} 23$ involves repeats beyond EC3.

3 To better understand the structural determinants of the heterotetrameric tip-link configuration and to determine

4 the cis configuration of PCDH15 upon complex formation with $\mathrm{CDH} 23$, we crystallized higher affinity

5 variants $^{36}$ of tip-link fragments containing repeats EC1 to EC3 of PCDH15 and EC1 to EC2 of CDH23. This

6 structure, comprising two molecules each of $h s$ PCDH15 EC1-3 G16D/N369D/Q370N in complex with $\mathrm{mm}$

7 CDH23 EC1-2 T15E, provides a complete view of the heterotetrameric tip-link bond (Fig. 2D). The two

8 PCDH15 protomers are seen in an $\mathrm{X}$ configuration flanking two molecules of $\mathrm{CDH} 23$, thereby forming a

9 "double handshake" consistent with the heterotetrameric model deduced from the hs PCDH15 EC2-3 structure

10 and independently proposed by Dionne et al. ${ }^{38}$ (Fig. 2 and Fig. S8), but with novel structural variations. The

11 structure is also compatible with longer fragments of CDH23 (Fig. S9). Interface areas for the two handshakes in

12 our structures are different, with one of them being larger than all the previously published heterodimeric

13 handshakes ${ }^{33}$ (Table S4). The aperture angle for the $h s$ PCDH15 dimer is $55.69^{\circ}$ in the tetramer, which is smaller

14 than observed for dimeric $m m$ PCDH15 EC1-3" and $h s$ PCDH15 EC2-3, implying that the "scissor" is less open

15 in the tip-link tetramer structure.

Interestingly, electron density quality is generally good throughout the heterotetrameric $h s$ PCDH15 EC1-3 G16D/N369D/Q370N + mm CDH23 EC1-2 T15E structure, except for parts of CDH23 EC2 repeats, which were not built ( $\beta$-strands F and G, Fig. S10A). This is also reflected in larger B factor values across the PCDH15 EC1 and $\mathrm{CDH} 23$ EC1-2 repeats, which suggest some inherent flexibility or disorder compared to other regions of the model (Fig. S10). Mutations G16D and T15E had been identified as enhancers of equilibrium binding for the $m m$ PCDH15 EC1-2 and $m m$ CDH23 EC1-2 complex $^{36}$, and may have helped stabilize a heterotetrameric complex that otherwise requires longer $\mathrm{CDH} 23$ fragments to form (mutations p.N369D and p.Q370N complete a canonical DXNDN $\mathrm{Ca}^{2+}$-binding motif at the end of PCDH15 EC3 and are not expected to affect structure in any way). Alternatively, the stoichiometry of the complex might not be uniform across the crystal lattice, yet a composite omit $2 \mathrm{mF}_{\mathrm{o}}-\mathrm{DF}_{\mathrm{c}}$ map generated with simulated annealing suggests that both $m m$ CDH23 EC1-2 protomers are present in the crystal (Fig. S10B,C). 
2 Some details of the handshake interactions seen in our heterotetrameric $h s$ PCDH15 EC1-3

$3 \mathrm{G} 16 \mathrm{D} / \mathrm{N} 369 \mathrm{D} / \mathrm{Q} 370 \mathrm{~N}+m m \mathrm{CDH} 23 \mathrm{EC} 1-2 \mathrm{~T} 15 \mathrm{E}$ structure (Fig. 2D,E) are subtly different when comparing to

4 the single handshake $m m$ PCDH15 EC1-2 + CDH23 EC1-2 complex ${ }^{33}$ (PDBs: 4APX and 4AXW in form I;

5 4AQ8 in form II). In both PCDH15 protomers of the heterotetrameric structure, the side chain of residue p.R113,

6 known to be important for the hetero trans tip-link bond and for inner-ear function ${ }^{21,32,33,71}$, interacts with

7 CDH23's p.Q98 rather than with CDH23's p.E77 (Fig. S11A). A similar arrangement was seen in the structure

8 of $m m$ PCDH15 EC1-2 isoform CD1-2 bound to $m m$ CDH23 EC1-2 ${ }^{37}$ (PDB: 4XXW). In addition, the CDH23

9 EC1 repeat of one of the protomers is closer to its PCDH15 EC2 partner (Fig. S11A,B), with PCDH15 EC1-2

10 and CDH23 EC1-2 inter-repeat angles varying across chains and structures (Fig. S8), although this flexing has

11 been observed across previously published structures of the $m m$ PCDH15 EC1-2 + CDH23 EC1-2 complex ${ }^{33}$.

In addition, in our heterotetramer structure, the $\alpha$ helices between $\beta$-strands $\mathrm{C}$ and $\mathrm{D}$ of CDH23 EC1 repeats are significantly closer to each other in the crystal structure (Fig. S11C) as compared to the heterotetramer model generated from our $h s$ PCDH15 EC2-3 dimer and to the one generated by Dionne et al. ${ }^{38}$ Mismatched species (mouse versus human CDH23 EC1-2 differing at p.R35Q, p.P153Q, p.Q168R, and p.V174T) and engineered mutations are unlikely to explain this difference. Instead, electrostatic interactions among highly conserved charged residues from both $\mathrm{CDH} 23$ protomers favor a close contact in this region. Residue p.E49 from one CDH23 protomer "caps" the N-terminus of the neighboring CD $\alpha$ helix in the opposite protomer. This conspicuous long-range helix capping ${ }^{72}$ appears to stabilize the overall structure of the heterotetramer. Furthermore, p.E50 from one $\mathrm{CDH} 23$ protomer forms a salt bridge with p.R53 of the neighboring $\mathrm{CDH} 23$ and vice versa (Fig. S11C). Thus, the less open conformation of the PCDH15 "scissor" squeezes the CDH23 protomers together, creating a $\mathrm{CDH} 23-\mathrm{CDH} 23$ interface with favorable contacts among the two protomers, which in turn may stabilize the overall heterotetrameric structure. Taken together, our structures and data strongly support parallel dimerization of PCDH15 at the N-terminal end mediated by EC2-3 repeats and tip-link bonds forming a heterotetramer with two PCDH15 EC1-2 + CDH23 EC1-2 handshakes (Fig. 2 and Figs. S8- 


\section{Structures of PCDH15's ectodomain middle region reveal atypical flexible linkers}

3 The middle region of PCDH15, encompassing repeats EC4 to EC8, has been less thoroughly explored than other parts of this protein. A previous structure of $h s$ PCDH15 EC3-5 CD1-1 (PDB: 5T4M) revealed two bound $\mathrm{Ca}^{2+}$

5 ions at the EC3-4 linker region and a canonical $\mathrm{Ca}^{2+}$-binding site at the EC4-5 linker region ${ }^{44}$. Protomers in the asymmetric unit displayed different orientations of EC3 with respect to EC4, and this flexibility was also evident

7 in simulations of this fragment ${ }^{44}$. However, this $h s$ PCDH15 EC3-5 CD1-1 structure lacked exon 12a, which encodes for a seven residue insertion p.V(414+1)PPSGVP(414+7) near the EC3-4 linker region. Given that structural variations due to isoform specific (in-frame) insertions or deletions might be relevant for function, we sought to obtain a structure that carries exon $12 \mathrm{a}$, present in some of the CD2 isoforms thought to be essential

11 for inner-ear mechanosensation ${ }^{20,73,74}$. We solved the hs PCDH15 EC3-5 CD2-1 structure, which contains three molecules in the asymmetric unit having distinct EC3-4 inter-repeat conformations and with two of the linkers with only one bound $\mathrm{Ca}^{2+}$ ion at site 3 (Fig. 3A-E). The insertion enlarges the EC4 BC loop, which projects away from the repeat without altering its folding but may affect $\mathrm{Ca}^{2+}$-binding affinity (Fig. 3B and Fig. S4C). Only the most bent conformation of this isoform is compatible with the antiparallel PCDH15 EC1-3 interface (Fig. S5), while all protomer conformations observed in the structure are compatible with the PCDH15 X-dimer mediated by EC2-3 (Fig. S12A). Overall, our mm PCDH15 EC3-5 CD2-1 structure is consistent with enhanced flexibility at the EC3-4 linker.

Another atypical linker region is expected in PCDH15 EC5-6: Sequence alignments show that EC5 has a modified XEX motif (p.499TDM501) and lacks the DRE motif (p.559ALT561) present in canonical $\mathrm{Ca}^{2+}-$ binding linker regions (Fig. S1). In addition, the DXNDN linker is p.586PPNNQ590 in EC5-6, but motifs involved in $\mathrm{Ca}^{2+}$ binding at site 3 (top of EC6) are mostly unchanged (DXD is p.621DRE623 and XDX is p.670SDG672). Two of our crystal structures cover the EC5-6 linker, which is seen in three different conformations (one in $\mathrm{mm}$ PCDH15 EC4-7 and two in $\mathrm{mm}$ PCDH15 EC5-7 I582T; Fig. 3F and Fig. S4D). In all 
1 S12D). In contrast, structures of $\mathrm{mm}$ PCDH15 EC6-7 and $\mathrm{mm}$ PCDH15 EC7-8 V875A show canonical, rather

2 straight linkers with three bound $\mathrm{Ca}^{2+}$ ions (variants $\mathrm{I} 582 \mathrm{~T}$ and V875A are not expected to alter structures).

3 While the two PCDH15 EC5-7 protomers in the asymmetric unit form a compelling parallel cis dimer in the $\mathrm{mm}$

4 PCDH15 EC5-7 I582T crystal structure (interface area of $1049.5 \AA^{2}$; Fig. S12B,C), all our fragments covering

PCDH15 EC4-7, EC5-7, and EC6-7 were monomeric in solution, as also reported for similar fragments by

6 Dionne et $\mathrm{al}^{38}$.

8 Overall, our structures of the middle region of PCDH15 suggest flexibility and altered $\mathrm{Ca}^{2+}$ binding at EC3-4 and EC5-6 linkers. Furthermore, three alternate conformations for each of the EC3-4 and EC5-6 linker regions assembled in an X-dimer mediated by EC2-3 give rise to 45 unique possible combinations of PCDH15 protomers (Fig. S12D). Some of these conformations display a remarkable separation between EC7 repeats (up to $26.8 \mathrm{~nm}$ ), compatible with a PCDH15-PCDH15 trans X-dimer (Fig. S6A). Importantly, given prior simulations of the EC3-4 fragment ${ }^{44}$ and the architecture of EC5-6, flexibility at these linker regions will occur even at high $\mathrm{Ca}^{2+}$ concentrations, with a large number of structurally diverse configurations facilitated by the EC2-3 X-dimer.

\section{Structures of PCDH15's ectodomain C-terminal fragments reveal kinks and cis dimerization}

Three previous studies reported high-resolution structures covering parts of the C-terminal end of PCDH15 including EC9 to MAD12 ( $h s$ PCDH15 EC8-10, $\mathrm{mm}$ PCDH15 EC9-10, ss PCDH15 EC10-MAD12, and $\mathrm{mm}$ PCDH15 EC11-MAD12) $)^{39,40,43}$. These structures revealed a semi-canonical EC8-9 linker region, a flexible, $\mathrm{Ca}^{2+}$ free and bent EC9-10 linker region, a canonical EC10-11 linker region, and the L-shaped arrangement of a ferredoxin-like MAD12 tucked against EC11, with EC11-MAD12 inducing parallel dimerization ${ }^{38-40}$. Our structure of $m m$ PCDH15 EC9-MAD12 (Fig. 4A,B) displays the bent EC9-10 linker region (as observed in $h s$ PCDH15 EC8-10 and $\mathrm{mm}$ PCDH15 EC9-10, albeit with a different azimuthal angle) ${ }^{43}$ and the EC10-MAD12 dimerization domain, both in one symmetric dimer (one molecule per asymmetric unit). The total dimer interface surface area is $1,267.9 \AA^{2}$ (including contacts between MAD12 and EC11 and between EC10 repeats; 
$1\left(\sim 1,1160 \AA^{2}\right.$, two molecules per asymmetric unit), with the interface area at the EC10-EC10 contact being

2 slightly larger ( $\sim 336 \AA^{2}$ compared to $\sim 185 \AA^{2}$ for $\left.6 \mathrm{BXZ}\right)$. The $m m$ PCDH15 EC9-MAD12 structure confirms

3 that dimerization is induced by EC11-MAD12 and that it is compatible with a kinked EC9-10.

5 Glycosylation is observed in our $\mathrm{mm}$ PCDH15 EC9-MAD12 structure at five sites, with O-linked sugars clearly

6 discernible at residues p.S996, p.S998, and p.T1000 in EC9, and N-linked sugars at p.N1063 in EC10 and at

7 p.N1154 in EC11 (Fig. 4C-E and Fig. S13). Interestingly, glycosylation has also been observed in published

8 structures at p.N1063 and p.N1154, but not at the other three sites ${ }^{39}$. Clear electron density at additional

9 glycosylation sites p.N1043 and p.S1141 (as seen in PDBs: 6C10 and 6C13 ${ }^{39}$ ) is not present in our structure.

10 While glycosylation at p.S1141 would sterically hinder the dimeric interface in one of the published PCDH15

11 structures (PDB: 6C13), none of the other modeled sugars in all available structures that cover this region

12 interfere with bending and dimerization directly.

14 Intriguingly, bending at the EC9-10 linker region in the context of the dimer positions EC9 repeats pointing in opposite directions, with the projections along their longest principal axis being parallel to a hypothetical membrane plane (Fig. S6D). Data from size exclusion chromatography coupled to multi-angle light scattering (SEC-MALS) confirmed that this protein fragment is dimeric in solution (Fig. S7B and Table 2), and the EC910 "kink" is also consistent with our previous structures ${ }^{43}$ and with low-resolution cryo-EM images of a PCDH15 fragment encompassing EC8 down to its transmembrane helix ${ }^{39}$. Small angle X-ray scattering (SAXS) data obtained using bacterially produced $\mathrm{mm}$ PCDH15 EC9-MAD12 are also consistent with a dimeric arrangement in solution, with molecular weight estimates of $98.12 \mathrm{kDa}$ and a $5.7 \%$ discrepancy with the sequence-derived molecular weight of $51.98 \times 2=103.06 \mathrm{kDa}$ (Fig. 4F-J, Table S5).

SAXS data can also provide information about the size and shape of a protein in solution. The radius of gyration $\left(R_{\mathrm{g}}\right)$ of bacterially produced $m m$ PCDH15 EC9-MAD12 from Guinier $\left(R_{\mathrm{g}}=46.72 \pm 2.94 \AA\right)$ and SAXS profile analyses $\left(R_{\mathrm{g}}=47.28 \pm 0.24 \AA\right.$ with maximum dimension $\left.D_{\max }=160 \AA\right)$ are in excellent agreement with each 
1 suggest that the overall shape of the dimer observed in the crystallographic structure is maintained in solution.

2 However, comparison of the SAXS data to X-ray intensities modeled from the $m m$ PCDH15 EC9-MAD12

3 crystal structure revealed some discrepancies reflected by a large $\chi^{2}$ value obtained for the fitting $\left(\chi^{2}=6.52\right)$ and

4 by a clear dip in the $q$-region $0.1-0.25 \AA^{-1}$ observed in the modeled intensities but absent in the experimental 5 data (Fig. 4F). X-ray intensities calculated from models obtained using a normal-mode analysis $\left(\right.$ SREFLEX $\left.^{75}\right)$ fit

6 the experimental data better than the crystal structure $\left(\chi^{2}=1.38\right)$ and lack the dip in the $q$-region $0.1-0.25 \AA^{-1}$

7 (Fig. 4F). The best model shows changes in orientation and rotation of EC9 along with loss of symmetry (Fig.

$84 \mathrm{~J})$, consistent with asymmetry observed in SAXS-derived models of ss PCDH15 EC10-MAD12 ${ }^{40}$. Analysis of

9 the Kratky plot (Fig. 4I) indicates that while the protein is folded in solution, there is significant flexibility.

Overall, SAXS data strongly supports a dimeric conformation of PCDH15 EC9-MAD12 that is asymmetric and

11 flexible in solution.

13 Conformational transitions that straighten the EC9-10 linker, observed in simulations ${ }^{43}$ and in cryo-EM data ${ }^{39}$, strongly suggest that bending and unbending is relevant for tip-link assembly and function. The variety of angles that EC9-10 may adopt in solution ${ }^{39}$, along with dimerization of PCDH15 at EC2-3 and MAD12, and the asymmetry and flexibility suggested by SAXS data offer an interesting set of arrangements for PCDH15 + CDH23 filaments that might be adopted by tip-links and kinociliary links ${ }^{14,76,77}$ (Fig. S6E). In addition, flexing at EC3-4 (Fig. 3), EC5-6 (Fig. S12), and at EC9-10 (Fig. 4) seems to be a basic geometric requirement for establishing a parallel PCDH15 cis dimer compatible with dimerization points mediated by both EC2-3 and EC11-MAD12.

The structures and models discussed above depict parts of the entire ectodomain of PCDH15, and only small fractions of the entire tip link. Although each of them provides valuable insight into PCDH15 function, the assembly of PCDH15 filaments and force transduction mediated by tip links, which must withstand forces ranging from 10 to $100 \mathrm{pN}^{47,48,78,79}$, requires an understanding of the structure, dynamics, and elastic response of the entire PCDH15-CDH23 complex. Therefore, we used our structures to build, simulate, and computationally 
1 PCDH15 EC1-5 + CDH23 EC1-3 bonds, and two independent complete dimeric PCDH15 EC1-MAD12 models bound to $\mathrm{CDH} 23 \mathrm{EC} 1-2$ or $\mathrm{CDH} 23 \mathrm{EC} 1-3$.

\section{Structural models and predicted elasticity of the complete PCDH15 ectodomain monomer}

To gain insights into the function and elastic behavior of PCDH15, and as similarly done for parts of CDH23 ${ }^{45}$

CDH23 EC1-2 (p.Q1 to p.D105) using the original handshake interaction ${ }^{33}$ as a template (PDB: 4APX; Fig. 5A,

Fig. S14, and Table S6; 172 kDa for the complex). The second model was built to get the mm PCDH15 EC1-

MAD12 CD2-1 WT protein ectodomain (p.D5 to p.S1341(+7) carrying the p.I582(+7)T and p.V875(+7)A variations) coupled to a model of $\mathrm{mm} \mathrm{CDH} 23 \mathrm{EC} 1-3$ (p.V2 to p.D317) using the handshake interaction observed in the $h s$ PCDH15 EC1-3 G16D/N369D/Q370N + mm CDH23 EC1-2 T15E structure (Fig. 5B, Fig. S15, and

Table S6; 184 kDa for the complex). These PCDH15 models allowed us to visualize three in-frame deletion segments and fifteen sites of missense mutations implicated in inherited deafness (Fig. 5A-J and Table S7), thus providing a structural framework to propose mechanisms underlying dysfunction of PCDH15 (see Supplementary Discussion).

Our models of the PCDH15 ectodomain adopt conformations dictated by the relative orientation (tilt and azimuthal angles) of successive EC repeats observed in various crystallographic structures, including the EC9-10 kink observed in $h s$ PCDH15 EC9-MAD12 ${ }^{43}$ (used for $h s$ PCDH15 EC1-MAD12 CD1-1) and in $m m$ PCDH15 EC9-MAD12 (used for $\mathrm{mm}$ PCDH15 EC1-MAD12 CD2-1). These may represent only some of the possible conformations adopted in solution and might not be representative of states under physiological tension as experienced by hair-cell tip links ${ }^{78,79}$. Short equilibrations followed by SMD simulations of the entire $h s$ and $m m$ PCDH15 ectodomain monomers bound to the tips of $\mathrm{CDH} 23$ and with all their $\mathrm{Ca}^{2+}$-binding sites occupied, allowed us to explore their conformational dynamics and elasticity in silico. Constant-velocity stretching of the 
1 the structure (Fig. 5K, Fig. S16A, Table 3, and Table S8) in a first phase characterized by a soft effective spring

2 constant $\left(k_{1} \sim 3.3 \mathrm{mN} / \mathrm{m}\right.$; Fig. $\left.5 \mathrm{~L}\right)$, followed by stretching of the EC5-6 and EC9-10 linker regions, ensued by

3 stretching of the entire chain $\left(k_{2} \sim 24.4 \mathrm{mN} / \mathrm{m}\right)$. Subsequent unrolling and then unfolding of MAD12 at a force

4 peak of $\sim 331 \mathrm{pN}$ happened without unbinding of CDH23 EC1-2 from PCDH15. The elastic response of $\mathrm{mm}$

PCDH15 EC1-MAD12 CD2-1 + CDH23 EC1-3 at $0.1 \mathrm{~nm} / \mathrm{ns}$ was very similar to that of the human complex $\left(k_{1}\right.$ $\sim 1.5 \mathrm{mN} / \mathrm{m}, k_{2} \sim 27.5 \mathrm{mN} / \mathrm{m}$, unfolding peak at $\sim 356 \mathrm{pN}$; Fig. S16B-C), suggesting that, at the stretching speeds used in our simulations, unfolding of MAD12 occurs before unbinding.

The soft elasticity phase observed during stretching simulations of the monomeric PCDH15 EC1-MAD12 $\mathrm{CD} 1 / 2-1+\mathrm{CDH} 23 \mathrm{EC} 1-2 / 3$ complexes stems mainly from unbending of the $\mathrm{Ca}^{2+}$-free $\mathrm{EC} 9-10$ linker region, as previously predicted ${ }^{43}$, and from stretching of EC5-6 and various other linkers. The predicted spring constants at $0.1 \mathrm{~nm} / \mathrm{ns}$ are an upper limit, as this stretching speed is one order of magnitude larger than physiological speeds of the basilar membrane ${ }^{81}$ at loud, high-frequency sound and drag might be reduced at slower speeds. Similarly, unfolding forces of MAD12 at $0.1 \mathrm{~nm} / \mathrm{ns}$ represent an upper bound as simulations and experiments at slower stretching speeds (loading rates) will naturally report smaller forces ${ }^{82,83}$. The elastic response of MAD12 in the context of stretching simulations of the monomeric $h s$ and $m m$ PCDH15 EC1-MAD12 CD1/2-1 + CDH23 EC12/3 complexes is consistent with simulations stretching the dimeric $m m$ PCDH15 EC9-MAD12 (Fig. S17) and our previous simulations of ss PCDH15 EC10-MAD12 ${ }^{40}$. Our new simulations including the entire PCDH15 ectodomain predict that MAD12 will unfold before unbinding of the cadherin handshake at fast stretching speeds.

\section{Structural models and predicted strength of the heterotetrameric PCDH15 - CDH23 bond}

To better understand the behavior of the handshake bond in the context of the heterotetrameric arrangement predicted from our $h s$ PCDH15 EC2-3 structure and observed in the $h s$ PCDH15 EC1-3 G16D/N369D/Q370N + mm CDH23 EC1-2 T15E complex, we built two model systems for simulation (Fig. 6A and Fig. S18A,C). The first one includes two molecules of $h s$ PCDH15 EC1-5 CD1-1 (p.Q1 to p.P587) forming an X-dimer (as seen in 
1 as seen in the $m m$ PCDH15 EC1-2 + CDH23 EC1-2 complex [4APX] $]^{33}$ and mutated to match the human sequence). The second system includes two molecules of $\mathrm{mm}$ PCDH15 EC1-5 CD2-1 (p.Q1 to p.L585(+7)) forming an X-dimer coupled to two molecules of $m m$ CDH23 EC1-3 (p.Q1 to p.D317) with the cis and trans interactions based on the $h s$ PCDH15 EC1-3 G16D/N369D/Q370N $+m m$ CDH23 EC1-2 T15E tetrameric structure. In both models, referred to here as $h s$ and $m m(\text { PCDH15 EC1-5 })_{2}+(\mathrm{CDH} 23 \mathrm{EC} 1-2 / 3)_{2}$, all linkers and repeats were in conformations obtained from crystal structures and all $\mathrm{Ca}^{2+}$-binding sites were occupied. To

7 mimic possible low $\mathrm{Ca}^{2+}$ concentration conditions in the cochlea, three additional models of the $h s$ (PCDH15 $\mathrm{EC} 1-5)_{2}+(\mathrm{CDH} 23 \mathrm{EC} 1-2)_{2}$ tetramer were built with two, one, or zero $\mathrm{Ca}^{2+}$ ions per linker, respectively (Table 3, Supplementary Discussion, and Table S8). All systems, saturated and not saturated with $\mathrm{Ca}^{2+}$, were equilibrated and then stretched at various speeds to test their stability and elasticity in silico.

Equilibration trajectories of the $h s$ and $m m(\mathrm{PCDH} 15 \mathrm{EC} 1-5)_{2}+(\mathrm{CDH} 23 \mathrm{EC} 1-2 / 3)_{2}$ tetramers saturated with $\mathrm{Ca}^{2+}$ revealed a tendency for the PCDH15 EC5 ends to get closer to each other within $10 \mathrm{~ns}$ (from $\sim 16 \mathrm{~nm}$ in $h s$ and $\sim 12 \mathrm{~nm}$ in $m m$ to $\sim 10 \mathrm{~nm}$ in both). The $h s \mathrm{CDH} 23 \mathrm{EC} 1-2$ protomers also get close to each other, mimicking the arrangement seen in the $h s$ PCDH15 EC1-3 G16D/N369D/Q370N + mm CDH23 EC1-2 T15E structure, where $\beta$-strands $\mathrm{A}$ and $\mathrm{G}$ of EC2 from one protomer get closer to the same strands in EC2 from the adjacent protomer. A similar arrangement was also seen in the equilibration of the $m m$ complex model. However, the p.E50:p.R53 salt bridge between adjacent CDH23 protomers (as seen in the crystal structure of the heterotetramer bond) did not form in the $h s$ complex, and $m m$ CDH23 EC3 protomers drifted away from each other. Moreover, the PCDH15 p.R113 salt bridge with CDH23 p.E77, absent in the $\mathrm{mm}$ model, was formed throughout its equilibration trajectory. These rearrangements highlight the dynamic nature of the heterotetrameric complex. The double handshake bond, however, along with the PCDH15 EC2-3 interface for parallel dimerization, remained stable during these short equilibrations.

Systems with less $\mathrm{Ca}^{2+}$ at all linkers exhibited two distinct behaviors in our equilibrium simulations. The system with $2 \mathrm{Ca}^{2+}$ ions per linker was stable, with the two PCDH15 EC5 protomers coming as close as $\sim 9 \mathrm{~nm}$ in a 
1 saturated linkers $\left(3 \mathrm{Ca}^{2+}\right.$ ions per linker, except for PCDH15 EC2-3 and EC3-4). More dramatic conformational

2 changes, including bending and twisting of linkers, were observed for systems with 1 and $0 \mathrm{Ca}^{2+}$ ions per linker,

3 especially at the PCDH15 EC3-4 linker regions. The conformational changes observed with less $\mathrm{Ca}^{2+}$, resulting

4 in overall shrinkage of the end-to-end distance of the complex, are consistent with results from simulations of

5 classical $^{84-86}$ and tip-link cadherins ${ }^{33,42,44}$ that in the absence of bound $\mathrm{Ca}^{2+}$ ions exhibited enhanced hinge-like

6 flexibility at their linker regions. These results are also consistent with EM images showing curled and collapsed

7 classical $^{87}$ and tip-link ${ }^{21}$ cadherins in the absence of $\mathrm{Ca}^{2+}$.

9 Stretching of the equilibrated $h s$ and $m m(\mathrm{PCDH} 15 \mathrm{EC} 1-5)_{2}+(\mathrm{CDH} 23 \mathrm{EC} 1-2 / 3)_{2}$ tetramers saturated with $\mathrm{Ca}^{2+}$ 10 at the constant speeds tested $(10,1$, and $0.1 \mathrm{~nm} / \mathrm{ns})$ revealed unbinding of the CDH23 EC1-2/3 protomers from 11 PCDH15 without unfolding of EC repeats (Fig. 6A and Fig. S18A-C). At the slowest stretching speed (0.1 $12 \mathrm{~nm} / \mathrm{ns})$, the $h s(\mathrm{PCDH} 15 \mathrm{EC} 1-5)_{2}+(\mathrm{CDH} 23 \mathrm{EC} 1-2)_{2}$ complex straightened, with PCDH15 EC4-5 repeats 13 establishing close contacts in a parallel arrangement. The "open scissor" conformation of the PCDH15 EC1-3 14 dimer closed itself as the stretching proceeded, thereby squeezing the CDH23 EC1-2 protomers together. As a 15 result, an intricate network of hydrogen bonds developed between conserved CDH23 EC1 residues. Further, a 16 rotation of the $\mathrm{CDH} 23 \mathrm{EC} 1-2$ protomers (Fig. 6 and Fig. S18A) preceded unbinding at large force $(\sim 1080 \mathrm{pN}$, 17 simulation S3d), which was roughly three times as large as the force observed during unfolding of MAD12 in 18 the monomeric $h s$ PCDH15 EC1-MAD12 + CDH23 EC1-2 complex ( $330 \mathrm{pN}$, simulation S1d), and $\sim 1.5$ times 19 larger than the force predicted for the unbinding of two non-interacting parallel handshakes $(\sim 720 \mathrm{pN}$ in 20 Sotomayor et al. $\left.{ }^{33}\right)$. Similarly, stretching of the $m m(\mathrm{PCDH} 15 \mathrm{EC} 1-5)_{2}+(\mathrm{CDH} 23 \mathrm{EC} 1-3)_{2}$ complex at $0.1 \mathrm{~nm} / \mathrm{ns}$ 21 resulted in unbinding at a large force peak $(\sim 800 \mathrm{pN}$, simulation $\mathrm{S} 7 \mathrm{~d})$, with squeezing of the CDH23 EC1-3 protomers (Fig. S19A-B). Thus, force-induced "locking" of CDH23 may make the tip-link bond more resistant against unbinding. The PCDH15 EC2-3 X-dimer interface was distorted but not lost after unbinding. These results suggest that the mechanical strength of the tetrameric tip-link bond is bolstered by the PCDH15 EC2-3 X-dimer.

27 Stretching of the equilibrated $h s(\mathrm{PCDH} 15 \mathrm{EC} 1-5)_{2}+(\mathrm{CDH} 23 \mathrm{EC} 1-2)_{2}$ tetramers that were not saturated with 
$1 \mathrm{Ca}^{2+}$ at all linker regions revealed a more complex set of events during unbinding (Fig. 6B and Fig. S19A-B).

2 SMD simulations of the system with $2 \mathrm{Ca}^{2+}$ ions per linker at $0.1 \mathrm{~nm} / \mathrm{ns}$ revealed unbinding without unfolding

3 (force peak at $\sim 737 \mathrm{pN}$, simulation S4d) with complete separation of the PCDH15 EC2-3 X-dimer interface.

4 SMD simulations of the system with $1 \mathrm{Ca}^{2+}$ ion per linker region at $0.1 \mathrm{~nm} / \mathrm{ns}$ revealed sequential unbinding

5 (main force peak at $\sim 833 \mathrm{pN}$, simulation S5d) with severe stretching of linker regions. SMD simulations of the

6 system with $0 \mathrm{Ca}^{2+}$ ions per linker region at $0.1 \mathrm{~nm} / \mathrm{ns}$ revealed minor partial unfolding, rupture of the EC2-3 X-

7 dimer, and unbinding (force peak at $853 \mathrm{pN}$, simulation 6d). The starting states for SMD simulations of systems with 1 and $0 \mathrm{Ca}^{2+}$ ions per linker region were shorter due to bending of ECs with respect to each other, resulting in a longer stretching phase ( $<25 \mathrm{~nm}$ to $\sim 27.5 \mathrm{~nm}$ in Fig. $6 \mathrm{~B}$ and Fig. S19A) at low force with rather soft elasticity $\left(k_{1 \mathrm{Ca}} \sim 20.5 \mathrm{mN} / \mathrm{m}\right.$ and $\left.k_{0 \mathrm{Ca}} \sim 21.7 \mathrm{mN} / \mathrm{m}\right)$ dominated by unbending and straightening of the repeats.

11 Extension of the linkers between EC repeats at higher force resulted in a displacement of the force peak to higher end-to-end distances. Interestingly, the elasticity stemming from the EC repeats also progressively softened with lower number of $\mathrm{Ca}^{2+}$ ions at the linker (Fig. S19A).

Overall, our simulations of the heterotetrameric PCDH15 - CDH23 bond suggest that parallel dimerization and $16 \mathrm{Ca}^{2+}$ ions modulate the strength of the tip link connection, which might in turn determine whether unbinding happens before unfolding.

\section{Structural models and predicted elasticity of the complete PCDH15 ectodomain dimer}

Our structures and biochemical data obtained using various PCDH15 fragments indicate that there are two possible points of parallel dimerization (EC2-3 and EC11-MAD12, as also reported by Ge et al. ${ }^{39}$ and Dionne et al. ${ }^{38}$ ). Additional MALS experiments with the full-length ectodomain of $\mathrm{mm}$ PCDH15 EC1-MAD12 confirm that this fragment is a dimer that can withstand mild $\mathrm{Ca}^{2+}$-chelation with EDTA (Fig. S7C). However, atomistic models of the entire PCDH15 ectodomain seem to be incompatible with an arrangement in which both points of dimerization exist simultaneously (Fig. S6D), even when the PCDH15 EC9-10 linker unbends (Fig. S20A-E). 
1 dimeric PCDH15 complex as observed in tip links ${ }^{11,21}$, we did a systematic search of conformations obtained

2 from equilibrium and SMD simulations that could be assembled into structures compatible with both points of

3 dimerization (Fig. 7A,B and Fig. S20E-G).

5 The first tetramer model built contains the $h s$ (PCDH15 EC1-MAD12 CD1-1 $)_{2}+(\mathrm{CDH} 23 \text { EC1-2 })_{2}$ complex

6 (p.Q1 to p.I1342 and p.Q1 to p.D205 per monomer; $\sim 343 \mathrm{kDa}$ ), while the second contains the $\mathrm{mm}$ (PCDH15

7 EC1-MAD12 CD2-1 $)_{2}+(\mathrm{CDH} 23 \mathrm{EC} 1-3)_{2}$ complex (p.Q1 to p.S1341(+7) carrying the p.I582(+7)T and 8 p.V875(+7)A variations for PCDH15 and p.Q1 to p.D317 for CDH23; 369 kDa; Table S6). All EC repeats and 9 linker regions in these $h s$ and $m m(\mathrm{PCDH} 15 \mathrm{EC} 1-\mathrm{MAD} 12)_{2}+(\mathrm{CDH} 23 \mathrm{EC} 1-2 / 3)_{2}$ tetrameric models were 10 obtained from crystallographic conformations or coordinates from simulations of crystal structures (Fig. $11 \mathrm{~S} 20 \mathrm{~F}, \mathrm{G})$. In equilibrium simulations of the $h s$ heterotetrameric model, in which the PCDH15 EC9-10 segment is 12 initially straight (Fig. 7A and Fig. S20F), we monitored re-bending at this linker region, with one of the 13 PCDH15 protomers more kinked than the other (Fig. 7E). A similar asymmetric bent conformation is maintained 14 in an equilibration of the $m m$ model (Fig. 7B and Fig. S21A). These results are consistent with simulations of the 15 isolated PCDH15 EC8-10 fragment ${ }^{43}$ and suggest that the EC9-10 kink can exist within the context of the entire 16 PCDH15 homodimer.

Equilibrated states of both $h s$ and $m m(\mathrm{PCDH} 15 \mathrm{EC} 1-\mathrm{MAD} 12)_{2}+(\mathrm{CDH} 23 \mathrm{EC} 1-2 / 3)_{2}$ tetrameric complexes were further used for SMD simulations at various stretching speeds (Fig. 7C-E and Fig. S21). In the slowest simulations $(0.1 \mathrm{~nm} / \mathrm{ns})$ of the $h s$ complex, straightening by unbending of various linker regions (most notably the EC9-10 kink) was followed by elongation of the EC3-4, EC5-6, and EC9-10 linkers and subsequent partial unrolling and unfolding of both MAD12s at the C-terminal end. Unbinding of CDH23 from PCDH15 was not observed at the slowest stretching speeds used. The initial straightening phase had an effective spring constant of $k \sim 45 \mathrm{mN} / \mathrm{m}$, while unrolling and unfolding occurred at $\sim 510 \mathrm{pN}$, with a re-coil reflected in re-bending at the EC9-10 linker region (Fig. 7E, rightmost panel). A similar series of events was observed for the $m m$ complex when stretched at $0.1 \mathrm{~nm} / \mathrm{ns}$, with an initial straightening phase that had an effective spring constant of $k \sim 37$ 
complete PCDH15 ectodomain dimer is significantly stiffer than the monomeric PCDH15 ectodomain.

\section{DISCUSSION}

4 Our structures, biochemical data, and simulations provide an integrated atomistic view of the entire PCDH15

5 ectodomain and its connection to the $\mathrm{CDH} 23$ tip. We found two points of parallel dimerization for PCDH15 (at

6 EC2-3 and EC11-MAD12) and provide a detailed and unique view of the two trans PCDH15-CDH23

7 handshakes in the heterotetrameric bond facilitated by the PCDH15 EC2-3 X-dimer. Simulations suggest that

8 the two handshakes in the heterotetramer are squeezed together by closing of the X-dimer scissor under tension

9 that mimics physiological stimuli. This squeezing strengthens the heterotetrameric bond with unbinding forces

10 predicted to be on average $\sim 1.3$ times larger than the sum of unbinding forces for independent handshakes,

11 reminiscent of a catch-bond behavior ${ }^{88}$. Our structures also revealed three points of $\mathrm{Ca}^{2+}$-independent flexibility

12 for PCDH15 at linker regions EC3-4, EC5-6, and EC9-10. Simulations of the entire monomeric PCDH15

13 ectodomain bound to $\mathrm{CDH} 23 \mathrm{EC} 1-2 / 3$ revealed a soft elastic response dominated by these flexible points, while

14 the heterotetrameric complex (dimeric PCDH15 bound to two CDH23 monomers) was stiffer yet still presented

15 kinks at the PCDH15 EC9-10 linker region. Intriguingly, our simulations predict that PCDH15's MAD12 unrolls

16 and then would unfold before $\mathrm{CDH} 23$ unbinding at the stretching speeds tested. These results provide a

17 structural framework to compare and interpret complementary experimental results and to understand the

18 function of PCDH15 as a key component of the tip links that open inner-ear transduction channels.

20 Two recent studies ${ }^{38,39}$ have provided important insights into PCDH15's structure using alternate and complementary approaches to those used here. Medium resolution $(\sim 11 \AA \AA)$ cryo-EM images of PCDH15 EC8MAD12 and its transmembrane domain in complex with $\mathrm{TMHS}^{39}$ eloquently revealed a highly dynamic EC8-10 ectodomain with either one or both protomers displaying kinked EC9-10 conformations that are consistent with our EC9-MAD12 structure and simulations. In the images reported by Ge et al. ${ }^{39}$, about $20 \%$ of the recorded conformations showed a bent conformation at this linker, with the refined straight model $(\sim 14 \%$ of conformations) suggesting that bending-unbending transitions may play a role in tip-link mechanics ${ }^{43}$. However,

27 it was unclear whether kinks and spontaneous bending-unbending dynamics were compatible with the complete 
1 dimeric ectodomain, as repeats EC1-7 were missing. Elegant structural models from negative staining EM

2 images of longer PCDH15 ectodomain fragments (EC1-EC11 and EC1-MAD12) revealed flexural flexibility ${ }^{38}$

3 and were nicely consistent with parallel dimerization at both the EC2-3 contacts (confirmed by a high resolution

4 structure of PCDH15 EC1-3 $3^{38}$ ) and the EC11-MAD12 segments. However, reconstructions and averages may

5 have neglected significant bending and flexibility observed in raw images (Fig. S8 in ${ }^{38}$ ), and resolution of the

6 envelope $(\sim 20 \AA)$ was not enough to visualize details of the middle and C-terminal regions of PCDH15. Our

7 structures, models, and simulations, obtained independently from the two studies mentioned above, not only fill

8 in the missing pieces by providing a high-resolution and detailed view of the entire dimeric PCDH15

9 ectodomain, but also test its elasticity and dynamics in silico. Altogether, these data strongly support a model in

10 which PCDH15 has two points of cis dimerization (EC2-3 and EC11-MAD12) and flexible linker regions at

11 EC3-4, EC5-6, and EC9-10 that, along with unrolling of MAD12, may contribute to the overall elasticity of

12 PCDH15.

14 Exquisite single-molecule force spectroscopy experiments ${ }^{89}$ have also started to elucidate the elastic response of the PCDH15 monomer. In these experiments, the monomeric PCDH15 EC1-EC11 p.V250D protein fragment stretched in the presence of different $\mathrm{Ca}^{2+}$ concentrations $\left(3 \mathrm{mM}, 20 \mu \mathrm{M}\right.$, and no $\left.\mathrm{Ca}^{2+}\right)$ behaved as a soft entropic spring, with an effective spring constant that varied from $<0.5 \mathrm{mN} / \mathrm{m}$ to $\sim 6 \mathrm{mN} / \mathrm{m}$ before rupture. Three types of unfolding events (among many) were robustly identified, with extensions of $\sim 4 \mathrm{~nm}\left(\mathrm{~A}_{U}\right), \sim 15 \mathrm{~nm}\left(\mathrm{~B}_{U}\right)$, and $\sim 35 \mathrm{~nm}\left(\mathrm{C}_{\mathrm{U}}\right)$. The last type of unfolding event is consistent with rupture of an entire EC repeat. Comparison of these results with predictions from our simulations is difficult, as these experiments did not include CDH23 or MAD12 and were performed at slow stretching speeds equivalent to $1 \mathrm{~Hz}$, with an artificial constant force of 2-4 pN maintained between stretching cycles. However, our predictions of reduced unfolding force peaks at low $\mathrm{Ca}^{2+}$ concentrations for various cadherins ${ }^{33,42,85,90}$, including PCDH15 as presented here, are consistent with an increase in the number of PCDH15 unfolding events as observed in experiments upon $\mathrm{Ca}^{2+}$ depletion $^{89}$. In addition, the predicted effective spring constants for the PCDH15 EC9-10 fragment at $0.02 \mathrm{~nm} / \mathrm{ns}, k \sim 8.4 \mathrm{mN} / \mathrm{m}$, and for the heterodimeric PCDH15 EC1-MAD12 + CDH23 EC1-2 complex at $0.1 \mathrm{~nm} / \mathrm{ns}, k \sim 3 \mathrm{mN} / \mathrm{m}$, are within 27 the range of those measured experimentally ${ }^{89}$. Our simulations suggest that this soft elasticity stems from 
1 unbending of kinked linker regions (EC9-10) and elongation of flexible linkers (EC3-4 and EC5-6) in the

2 presence of saturating $\mathrm{Ca}^{2+}$ concentrations, rather than from each EC repeat behaving as an individual part of a

3 freely jointed chain ${ }^{89}$. Interestingly, lengthening of the heterodimeric PCDH15 EC1-MAD12 + CDH23 EC1-2

4 complex ( $\sim 15 \mathrm{~nm}$, Fig. 5 and Fig. S16) matches lengthening in $\mathrm{B}_{\mathrm{U}}$ events, but it is unclear why these continuous

5 transitions in simulations would appear as discrete length jumps in experiments. Importantly, the elastic response

6 of the monomeric PCDH15 ectodomain will be influenced by unrolling and unfolding of MAD12 ${ }^{40}$, not present

7 in the experiments. Since PCDH15 most likely functions as a cis dimer in the tip link ${ }^{21}$, the elastic response of

8 the entire dimeric PCDH15 ectodomain, including the mechanically weak MAD12, needs to be taken into 9 account.

11 Our simulations of the $h s$ and $m m(\mathrm{PCDH} 15 \mathrm{EC} 1-\mathrm{MAD} 12)_{2}+(\mathrm{CDH} 23 \mathrm{EC} 1-2 / 3)_{2}$ tetrameric complexes predict 12 a stiffer elastic response ( $k \sim 40 \mathrm{mN} / \mathrm{m}$ at $0.1 \mathrm{~nm} / \mathrm{ns}$ with saturating $\mathrm{Ca}^{2+}$ concentrations) than that monitored for 13 the monomer. Yet this estimate represents an upper bound, given the fast stretching speeds used in simulations 14 and the possibility that further equilibration of our models results in additional bending of EC9-10 and the other 15 flexible linker regions of PCDH15 (EC3-4 and EC5-6). A completely relaxed state with bent linkers will be softer than a pre-stretched initial conformation. Thus, our data suggests that the degree of bending of various linker regions in the initial state of the dimeric PCDH15 molecules, controlled in part by the $\mathrm{Ca}^{2+}$ concentration around it, will determine whether it behaves as a soft or stiff spring. Our data also suggests that dimerization of PCDH15 as well as binding to $\mathrm{CDH} 23$ will have a significant impact on the conformational space available to the PCDH15 monomers and their response to force upon tetramer formation.

Whether tip links made of PCDH15 and CDH23 act as soft gating springs or stiff cables conveying tension in 23 vivo has been an open question for decades ${ }^{47,91,92}$. SMD simulations of $m m \mathrm{CDH} 23 \mathrm{EC} 1-2$ saturated with $\mathrm{Ca}^{2+}$ 24 ions bound at its four sites (three at the EC1-2 linker and one at the EC1 tip) revealed a stiff fragment and large unfolding force peaks that were $\mathrm{Ca}^{2+}$-dependent. In absence of structural information from any other tip-link 26 fragment at the time, the tip link was suggested to be stiff under the assumption that all other EC repeats would 27 behave similarly ${ }^{42}$, yet further structural and computational work revealed flexible ${ }^{44}$ and elastic elements ${ }^{43}$ within 
1 PCDH15, and some atypical linkers in $\mathrm{CDH} 23^{45}$. These data suggested that a kinked PCDH15 EC9-10 could

2 provide $\mathrm{Ca}^{2+}$-independent elasticity and at the same time withstand the tip-link resting tension ${ }^{78}$, thus supporting

3 a model in which the lower end of the tip link formed by PCDH15 could be soft, while the upper part of the tip

4 link formed by $\mathrm{CDH} 23$ could be stiff ${ }^{43}$. Our data partially supports this model: the structure of $\mathrm{mm}$ PCDH15

5 EC9-MAD12 reveals that the EC9-10 kink is still present in the context of a longer construct, there are further

6 points of $\mathrm{Ca}^{2+}$-independent elasticity in PCDH15 (particularly stemming from EC5-6), and the monomeric

7 PCDH15 EC1-MAD12 is soft $(k \sim 3 \mathrm{mN} / \mathrm{m}$ at $0.1 \mathrm{~nm} / \mathrm{ns})$. However, while PCDH15 EC9-10 is still kinked in the

context of the PCDH15 cis dimer, our heterotetrameric models are significantly stiffer than the heterodimeric models $(k \sim 40 \mathrm{mN} / \mathrm{m}$ at $0.1 \mathrm{~nm} / \mathrm{ns})$. Unrolling and unfolding of MAD12 along with the $\mathrm{Ca}^{2+}$-dependent response

10 of various linker regions add another layer of complexity to the elastic response of PCDH15. The elastic 11 response of MAD12 might be modified by its interactions to TMHS $^{39}$ and other components of the transduction 12 apparatus that may help prevent unrolling and unfolding. At very low $\mathrm{Ca}^{2+}$ concentrations PCDH15 might 13 behave as a set of independent, freely jointed repeats ${ }^{85,89}$, with resting tension keeping these chains pre-stretched.

14 Thus, depending on binding partners and the conditions in which PCDH15 is found, it might behave as an elastic or stiff element (Fig. 8 and Fig. S22).

Biophysical experiments with bullfrog saccular hair cells stimulated ex vivo with a flexible fiber first identified

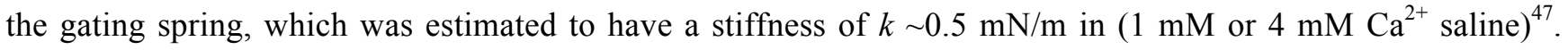
Similar experiments using an optical trap estimated the gating spring stiffness to be $k \sim 0.5 \mathrm{mN} / \mathrm{m}\left(4 \mathrm{mM} \mathrm{Ca}^{2+}\right.$ saline $)^{48}$, and a recent study using calibrated fluid-jet stimulation determined a gradient of gating spring stiffness along the mammalian cochlea ranging from $\sim 0.5 \mathrm{mN} / \mathrm{m}$ to $\sim 1.7 \mathrm{mN} / \mathrm{m}$ in inner hair cells and from $\sim 1.3 \mathrm{mN} / \mathrm{m}$ to $3.7 \mathrm{mN} / \mathrm{m}$ in outer hair cells (stiffness measurements done with $1.5 \mathrm{mM} \mathrm{Ca}^{2+}$ saline). Interestingly, the resting tension per tip link was also found to vary along the cochlea, from $\sim 5.9 \mathrm{pN}$ to $\sim 16 \mathrm{pN}$ for inner hair cells, and from $\sim 4.7 \mathrm{pN}$ to $\sim 34 \mathrm{pN}$ for outer hair cells. Maximal tip-link resting tension was estimated to be $\sim 50 \mathrm{pN}$. In all cases, the gating-spring stiffness and resting tension per tip link were indirectly estimated; we lack direct information on what forces each tip link is experiencing, whether resting tension is applied as a constant or 
stretching of tip link molecules in vivo.

3 Laser velocimetry experiments from chinchilla cochlear hair cells in vivo indicate that the basilar membrane can

4 move as fast as $0.01 \mathrm{~m} / \mathrm{s}$ for loud sound ( $>80 \mathrm{~dB}$ SPL $)^{81,93}$. Volumetric optical coherence tomography

5 vibrometry data from mouse ${ }^{94}$ is consistent with the chinchilla measurements and indicate that peak stereocilia

6 displacements in mice cochlear hair cells range between $\sim 10 \mathrm{~nm}$ to $\sim 150 \mathrm{~nm}$ in response to sounds that go from

$710 \mathrm{~dB}$ SPL $(\sim 6 \mathrm{kHz})$ to $80 \mathrm{~dB}$ SPL $(\sim 10 \mathrm{kHz})$ in vivo. These data are in agreement with in situ estimates of

8 mouse and guinea pig stereocilia displacements ${ }^{95,96}$, and suggest that stereocilia can move as fast as $0.0015 \mathrm{~m} / \mathrm{s}$

9 in normal hearing, and perhaps even faster for very loud sounds. Thus, stretching speeds used in our simulations

10 are about one order of magnitude faster than those experienced by tip links in vivo, yet there is abundant

11 theoretical $^{97-100}$, computational $^{61,82,101}$, and experimental work ${ }^{83}$ that can be used to interpret results at these high

12 force loading rates. As stretching speed and loading rates increase, unbinding and unfolding forces are naturally

13 larger, a phenomenon that is well documented in experiments ${ }^{83}$ and simulations ${ }^{82}$ and that is well understood

14 theoretically ${ }^{99,100}$. While we cannot rule out that the sequence and type of events (unfolding vs. unbinding)

15 observed at fast stretching speeds in simulations will be the same at slower stretching speeds, our simulations

16 were carried out at stretching speeds that are slower than those that may favor unfolding of terminal ends ${ }^{102}$ and

17 provide specific predictions that can be tested and challenged experimentally in vitro.

19 In vivo conditions for tip links vary significantly across organs and species. For instance, $\mathrm{Ca}^{2+}$ concentration near 20 tip links will greatly depend on the organ in which hair cells are located ${ }^{103}$. The vestibular endolymph $\mathrm{Ca}^{2+}$ 21 concentration is $>100 \mu \mathrm{M}$, while the bulk cochlear endolymph $\mathrm{Ca}^{2+}$ concentration varies from 20 to $40 \mu \mathrm{M}^{52,53}$.

22 Intriguingly, $\mathrm{Ca}^{2+}$ concentrations near hair bundles in the sub-tectorial space might be significantly higher than 23 previously thought $(>300 \mu \mathrm{M})$, both because of the action of stereocilia $\mathrm{Ca}^{2+}$ pumps functioning in a restricted 24 space and because of the buffering effect of the tectorial membrane ${ }^{54,55}$. Similarly, resting tension and 25 physiological forces will vary within and across organs, where glycosylation and differential expression of 26 various tip-link PCDH15 isoforms may also be diverse. The complexity of PCDH15's ectodomain revealed by 27 our structure and simulations and the various set of environments in which it functions in mechanotransduction 
1 indicate that PCDH15 might be a versatile and multimodal protein that can be tuned to display distinct elastic

2 responses (Fig. 8 and Fig. S22). While under some conditions PCDH15 can display the soft elasticity and

3 extensibility attributed to gating springs, directly determining the exact in vivo conditions in which PCDH15

4 functions remains a necessary and challenging step required to fully comprehend the role played by tip links in

5 inner-ear mechanotransduction.

7 PCDH15 is also found in auditory cortex interneurons ${ }^{104}$, eye photoreceptros ${ }^{71,105}$, and various other tissues ${ }^{23,106}$

where it may play a role in cell-cell adhesion and tissue development and maintenance. PCDH15's interaction

with $\mathrm{CDH} 23$ seems to be essential for auditory cortex wiring ${ }^{104}$, while its specific role in photoreceptor function

is less clear. Our PCDH15 structures and biochemical assays probing mutations that impair heterophilic binding

to $\mathrm{CDH} 23$, cis dimerization, and $\mathrm{Ca}^{2+}$ binding provide data that can inform the exploration of PCDH15's

12 function beyond inner-ear mechanotransduction.

METHODS

\section{Cloning, expression, and purification of bacterially expressed PCDH15 fragments}

DNA sequences encoding for protein fragments $m m$ PCDH15 EC1-2, $h s$ PCDH15 EC1-3, $h s$ PCDH15 EC1-4, $m m$ CDH23 EC1-2, $h s$ PCDH15 EC2-3, hs PCDH15 EC3-5, mm PCDH15 EC7-8, and mm PCDH15 EC9MAD12 were subcloned into NdeI and XhoI sites of the pET21a vector. Similarly, NheI and XhoI sites were used for fragments $\mathrm{mm}$ PCDH15 EC4-7, EC5-7, and EC6-7. All engineered missense mutations were generated using the QuikChange Lightning mutagenesis kit (Agilent). Insertions of a BAP sequence (p.GLNDIFEAQKIEWHE) at the end of $\mathrm{mm}$ PCDH15 EC1-2BAP and of exon 12a (p.VPPSGVP) in $h s$ PCDH15 EC3-5 CD2-1 were carried out using standard protocols. All DNA constructs were sequence verified.

24 Protein fragments were expressed in Escherichia coli BL21 (Agilent), BL21 CodonPlus(DE3)-RIPL (Agilent), or BL21 Rosetta(DE3) (Novagen) cells, which were cultured in LB or TB media, induced at $\mathrm{OD}_{600} \sim 0.6$ with $200 \mu \mathrm{M}$ or $1 \mathrm{mM}$ IPTG and grown at $30^{\circ} \mathrm{C}$ or $37^{\circ} \mathrm{C}$ for $\sim 16 \mathrm{hr}$ (Table S3). Cells were lysed by sonication in 
1 imidazole). The cleared lysates were loaded onto Ni-Sepharose (GE Healthcare), eluted with denaturing buffer

2 supplemented with $500 \mathrm{mM}$ imidazole and refolded as indicated in Table S3 using MWCO 2000 membranes

3 (Spectra/Por). Refolded protein was concentrated (Vivaspin or Amicon $10 \mathrm{kDa}$ ) and further purified on

4 Superdex S75 or S200 columns (GE Healthcare) in SEC buffer as indicated in Table S3. Pure fractions of $h s$

PCDH15 EC1-3 G16D/N369D/Q370N and $m m$ CDH23 EC1-2 T15E were mixed and purified in $20 \mathrm{mM}$ HEPES pH 7.5, $150 \mathrm{mM} \mathrm{KCl}$, and $2 \mathrm{mM} \mathrm{CaCl}_{2}$. All protein samples were concentrated by ultrafiltration to $>1$ $\mathrm{mg} / \mathrm{ml}$ for crystallization or biochemical assays.

\section{Cloning, expression, and purification of mammalian expressed PCDH15 fragments}

Mouse PCDH15 EC1-MAD12 CD1-1 (p.Q1 to p.G1327), EC1-3 (p.Q1 to p.N369), EC1-4 (p.Q1 to p.N491), and EC9-MAD12 (p.M905 to p.E1360) were subcloned into a pHis-N1 vector (a modified version of the pEGFP-N1 vector from Clontech where the EGFP has been substituted for a hexahistidine tag) using XhoI and

KpnI sites. The native signal sequence was included before the start of EC1 and EC9, mutation p.V250N was introduced in EC1-4 using the QuikChange Lightning mutagenesis kit (Agilent), and all constructs were sequence verified. All protein fragments were expressed by transient transfection of Expi293 cells using ExpiFectamine. After 4-5 days of expression, the conditioned media (CM) was collected and dialyzed overnight against $20 \mathrm{mM}$ TrisHCl, $\mathrm{pH} 7.5,150 \mathrm{mM} \mathrm{KCl}, 50 \mathrm{mM} \mathrm{NaCl}$, and $10 \mathrm{mM} \mathrm{CaCl}_{2}$ to remove EDTA. The CM was concentrated using Amicon $10 \mathrm{kD}$ or $30 \mathrm{kD}$ concentrators and incubated with Ni-Sepharose beads for $1 \mathrm{~h}$. The beads were washed 3 times with $20 \mathrm{mM}$ TrisHCl, pH 8.0, $300 \mathrm{mM} \mathrm{NaCl}(200 \mathrm{mM} \mathrm{NaCl}$ for $m m$ PCDH15 EC1MAD12), $10 \mathrm{mM} \mathrm{CaCl}$, and $20 \mathrm{mM}$ imidazole, and the target protein was eluted with the same buffer containing $500 \mathrm{mM}$ imidazole. The $\mathrm{mm}$ PCDH15 EC9-MAD12 protein was further purified on a Superdex S200 $\mathrm{mg} / \mathrm{mL}$ for crystallization. The $\mathrm{mm}$ PCDH15 EC1-3 and $m m$ PCDH15 EC1-4 (WT and p.V250N) protein 


\section{Crystallization, data collection, and structure determination}

2 Crystals were grown by vapor diffusion at $4^{\circ} \mathrm{C}$ by mixing protein and reservoir solutions as indicated (Table S2).

3 Cryoprotection buffers were prepared as indicated in Table S2. All crystals were cryo-cooled in liquid $\mathrm{N}_{2}$. X-ray

4 diffraction data was collected as indicated in Table 1 and processed with HKL2000 ${ }^{107}$. Structures were

5 determined by molecular replacement using PHASER ${ }^{108}$. The structure of $m m$ PCDH15 EC1-2BAP was solved

6 using repeats EC1-2 from $\mathrm{mm}$ PCDH15 in the handshake complex with $\mathrm{mm}$ CDH23 EC1-2 (4APX) ${ }^{33}$. The $\mathrm{mm}$

7 PCDH15 EC1-2 + CDH23 EC1-2 complex (4APX $\left.{ }^{33}\right)$ and the $h s$ PCDH15 EC2-3 V250N fragment (see below)

were used to solve the $h s$ PCDH15 EC1-3 G16D/N369D/Q370N structure, which was used along with the $m m$

PCDH15 EC1-2 + CDH23 EC1-2 complex (4AQ8) $)^{33}$ to solve the structure of $h s$ PCDH15 EC1-3

$10 \mathrm{G} 16 \mathrm{D} / \mathrm{N} 369 \mathrm{D} / \mathrm{Q} 370 \mathrm{~N}$ in complex with $\mathrm{mm} \mathrm{CDH} 23 \mathrm{EC} 1-2 \mathrm{~T} 15 \mathrm{E}$. Refinement of this heterotetrameric structure

11 used the amplitude-based "Twin Refinement" option in REFMAC5 after achieving an $R_{\text {free }}$ value of $\sim 32 \%$. The

12 hs PCDH15 EC2-3 WT structure was solved using repeat EC2 from $m m$ PCDH15 in the handshake complex

13 with $m m$ CDH23 EC1-2 (4APX) and EC3 from hs PCDH15 EC3-5 (5T4M), and subsequently used to solve for

$14 h s$ PCDH15 EC2-3 V250N. The $h s$ PCDH15 EC3-5 CD2-1 structure was solved using $h s$ PCDH15 EC3-5

$15\left(5 \mathrm{~T}_{4} \mathrm{M}^{44}\right)$. Refinement of this structure used the amplitude-based "Twin Refinement" option in REFMAC5 after

16 achieving an $R_{\text {free }}$ value of $\sim 27 \%$. Initial search models for $m m$ PCDH15 EC4-7 were individual EC4 and EC5

17 repeats from $h s$ PCDH15 EC3-5 $\left(5 \mathrm{~T}_{4} \mathrm{M}^{44}\right)$, and EC7 from $\mathrm{mm}$ PCDH15 EC7-8 V875A. The $m m$ PCDH15 EC5-

187 structure was solved using individual EC repeats from $\mathrm{mm}$ PCDH15 EC4-7. Mouse PCDH15 EC6-7 was

19 solved using EC7 from $\mathrm{mm}$ PCDH15 EC7-8 V875A as an initial search. The $\mathrm{mm}$ PCDH15 EC9-MAD12

20 structure was solved using ss PCDH15 EC10-MAD12 (6BXZ) ${ }^{40}$ and $h s$ PCDH15 EC8-10 (4XHZ) ${ }^{43}$. Model

21 building was done with $\mathrm{COOT}^{109}$ and restrained refinement was performed with REFMAC5 ${ }^{110}$ as indicated in

22 the deposited structures. Data collection and refinement statistics are provided in Table 1. Structures were further

23 analyzed using Procheck ${ }^{111}$, Whatcheck ${ }^{112}$, and Checkmymetal ${ }^{113}$ prior to deposition.

\section{SEC-MALS}

SEC-MALS experiments were done using an ÄKTAmicro system connected in series with a Wyatt miniDAWN 
column in $20 \mathrm{mM}$ TrisHCl, $\mathrm{pH} 8.0,150 \mathrm{mM} \mathrm{KCl}, 50 \mathrm{mM} \mathrm{NaCl}$ and $2 \mathrm{mM} \mathrm{CaCl}_{2}$. Bacterially produced protein samples of $h s$ PCDH15 EC1-3 and EC1-4 (WT and p.L306N/V307N mutants), as well as mammalian expressed protein fragments $m m$ PCDH15 EC1-3 and EC1-4 (WT and p.V250N), all at concentrations $>1 \mathrm{mg} / \mathrm{mL}$, were separated on a Superdex S200 3.2/3.0 column in $20 \mathrm{mM}$ TrisHCl, $\mathrm{pH}$ 8.0, $150 \mathrm{mM} \mathrm{KCl,} 50 \mathrm{mM} \mathrm{NaCl}$ and $2 \mathrm{mM}$ column in $20 \mathrm{mM}$ TrisHCl, $\mathrm{pH} 8.0,150 \mathrm{mM} \mathrm{KCl}$, with either $5 \mathrm{mM} \mathrm{CaCl}_{2}$ or $5 \mathrm{mM}$ EDTA. Absorbance at 280 $\mathrm{nm}$ and light scattering were monitored. The scattering information was subsequently converted into molecular weight using a rod-like model (Table 2). The SEC-MALS curves were adjusted for connecting tubing length before plotting. Measurements listed in Table 2 were taken from distinct samples.

\section{SEC-SAXS}

SEC-SAXS data was collected at the SIBYLS beamline 12.3.1 in the Advanced Light Source facility (Berkeley, CA) as described ${ }^{114,115}$ (Table S5). A bacterially produced and purified sample of $\mathrm{mm}$ PCDH15 EC9-MAD12 was used along with an Agilent 1260 series HPLC and a Shodex KW-803 analytical column for data collection at a flow rate of $0.5 \mathrm{ml} / \mathrm{min}$ at $20^{\circ} \mathrm{C}\left(20 \mathrm{mM}\right.$ TrisHCl pH $8.0,150 \mathrm{mM} \mathrm{KCl}, 5 \mathrm{mM} \mathrm{CaCl}_{2}$, and $\left.1 \mathrm{mM} \mathrm{TCEP}\right)$. Xray exposures lasting $3 \mathrm{~s}$ were collected continuously during a $\sim 40$ min elution. SAXS frames recorded prior to the protein elution peak were used to subtract all other frames. Buffer subtraction and data reduction was performed at the beamline with SCÂTTER ${ }^{116}$.

Further data analysis of the merged SAXS data was carried out with PRIMUS ${ }^{117}$ and the ATSAS program suite ${ }^{118}$. Estimates of the radius of gyration $\left(R_{\mathrm{g}}\right)$ from the Guinier region were measured with PRIMUS. Maximum dimension $\left(D_{\max }\right)$ of particles was estimated from an indirect Fourier transform of the SAXS profiles using $\mathrm{GNOM}^{119}$. Values of $D_{\max }$ between 140 and $160 \AA$ provided the best solutions. The oligomeric state of the server $^{120}$. Search of conformational changes of the dimeric structure in solution was performed by normal modes analysis with SRFLEX ${ }^{75}$ using the crystal structure of $m m$ PCDH15 EC9-MAD12 (PDB: 6EET) as starting 
1 the experimental SAXS data using FoXS ${ }^{121}$.

\section{AUC}

4 Sedimentation velocity AUC experiments were performed in a ProteomeLab XL-I analytical ultracentrifuge

5 (Beckman Coulter) following standard procedures ${ }^{122-124}$. Briefly, SEC purified protein samples were loaded into

6 AUC cell assemblies with Epon centerpieces and $12 \mathrm{~mm}$ path length. To achieve chemical and thermal

7 equilibrium, the An-50 TI rotor with loaded samples was allowed to equilibrate for $\sim 2 \mathrm{~h}$ at $20^{\circ} \mathrm{C}$ in the

8 centrifuge. The rotor was spun at 50,000 rpm and data was collected using absorption optics. Data analysis was

9 performed with the software SEDFIT (http://sedfitsedphat.nibib.nih.gov), using a continuous sedimentation

10 coefficient distribution model $c(S)$. Standard values for buffer viscosity $(0.01002$ poise $)$, density $(1 \mathrm{~g} / \mathrm{ml})$ and

11 partial specific volume $(0.73 \mathrm{ml} / \mathrm{g})$ were used, and confidence level was set to 0.68 as routinely done. The

12 obtained $c(S)$ distribution was loaded in GUSSI ${ }^{125}$. All experiments were done in duplicates from distinct 13 samples.

\section{Simulated systems}

16 The psfgen, solvate, and autoionize $\mathrm{VMD}^{126}$ plugins were used to build eleven molecular systems for simulations (Table 3 and Tables S6 and S8). The hs PCDH15 EC1-MAD12 + CDH23 EC1-2 model (1,547 residues, $\sim 171.8 \mathrm{kDa}$ ) was built from fragments $m m$ PCDH15 EC1-2 (4APX $\left.{ }^{33}\right), h s$ PCDH15 EC2-3 (chain D), $h s$ PCDH15 EC3-5 (5T4M ${ }^{44}$, chain A), $m m$ PCDH15 EC4-7, $m m$ PCDH15 EC7-8 V875A, $h s$ PCDH15 EC8-10

$\left(4 \mathrm{XHZ}^{43}\right)$, and $s s$ PCDH15 EC10-MAD12 $\left(6 \mathrm{BXZ}{ }^{40}\right.$, chain C) as indicated in Table S6 and Fig. S14. The $m m$

PCDH15 EC1-MAD12 CD2-1 + CDH23 EC1-3 model (1,665 residues, 184.4 kDa) was built from fragments

hs PCDH15 EC1-3 G16D/N369D/Q370N + mm CDH23 EC1-2 (chains B \& C, respectively), hs PCDH15 EC3-

5 CD2-1 (chain B), $m m$ PCDH15 EC4-7, $m m$ PCDH15 EC7-8 V875A, hs PCDH15 EC8-10 (4XHZ $\left.{ }^{43}\right), \mathrm{mm}^{2}$

PCDH15 EC9-MAD12, and $d r$ CDH23 EC1-3 (5W4T ${ }^{45}$, chain A) as indicated in Table S6 and Fig. S15. The $h s$

$(\mathrm{PCDH} 15 \mathrm{EC} 1-5)_{2}+(\mathrm{CDH} 23 \mathrm{EC} 1-2)_{2}$ model $(1,587$ residues, $\sim 176.6 \mathrm{kDa})$ was built from crystal structures of 
$1 h s$ PCDH15 EC1-3 G16D/N369D/Q370N + mm CDH23 EC1-2, $h s$ PCDH15 EC3-5 CD2-1 (chain B), and $d r$

$2 \mathrm{CDH} 23 \mathrm{EC} 1-3$ (5W4T ${ }^{45}$, chain A). All fragments were mutated so as to obtain a model of the species-specific

3 WT proteins (Table S6). The $h s(\mathrm{PCDH} 15 \mathrm{EC} 1-5)_{2}+h s(\mathrm{CDH} 23 \mathrm{EC} 1-2)_{2}$ systems with less $\mathrm{Ca}^{2+}$ ions at the

4 linker regions were built from the model with $\mathrm{Ca}^{2+}$-saturated linkers by removing ions sequentially while

5 maintaining charge neutrality.

7 The $h s(\mathrm{PCDH} 15 \mathrm{EC} 1-\mathrm{MAD} 12)_{2}+(\mathrm{CDH} 23 \mathrm{EC} 1-2)_{2}$ tetramer system was assembled from the coordinates obtained from simulation trajectories as follows (Fig. S20F). Chain A of the $h s$ PCDH15 used the coordinates from simulation S3c for residues 1-238 (chain A at $6.17 \mathrm{~ns}$ ), whereas residues $239-485$ come from simulation

$10 \mathrm{~S} 3 \mathrm{c}$ (chain B at 6.17 ns), residues 486-1228 come from simulation S1d (118.342 ns), and residues 1229-1342 11 come from simulation S11c (chain A at $2.88 \mathrm{~ns}$ ). Chain B of the $h s$ PCDH15 used the coordinates from 12 simulation S3c for residues 1-367 (chain B at $6.17 \mathrm{~ns}$ ), whereas residues 368-901 come from simulation S1d 13 (7.057 ns), residues 902-1003 come from simulation S1d (aa8.332 ns), residues 1004-1126 come from 14 simulation S1d (117.069 ns), and residues 1127-1342 come from simulation S11c (chain B at $2.88 \mathrm{~ns}$ ). The $h s$ 15 (CDH23 EC1-2) $)_{2}$ fragment comes from simulation S3c (chains C \& D at $\left.6.17 \mathrm{~ns}\right)$.

The $m m(\text { PCDH15 EC1-MAD12 })_{2}+(\mathrm{CDH} 23 \mathrm{EC} 1-3)_{2}$ tetramer system was assembled from the coordinates obtained from simulation trajectories and crystal structures as follows (Fig. S20G). Residues 3-116, 125-164, and 182-199 of CDH23 EC1-3 come from the crystal structure of $h s$ PCDH15 EC1-3 G16D/N369D/Q370N + $m m$ CDH23 EC1-2 (chain C), residues 114-121(+3), 162-178(+3), and 197-315(+3) come from the crystal structure of $d r \mathrm{CDH} 23 \mathrm{EC} 1-3$ (5W4T ${ }^{45}$; chain A). Residues 1-359 of chain A in the $m m$ PCDH15 EC1-MAD12 model come from the crystal structure of $h s$ PCDH15 EC1-3 G16D/N369D/Q370N + mm CDH23 EC1-2 (chain A), whereas residues 360-493 come from simulation S7b (chain A at 0.19 ns). Residues 494-600 in chain A of PCDH15 come from the equilibration simulation S2a at $2.502 \mathrm{~ns}$, whereas residues 601-913 come from the constant velocity SMD simulation S2c at $1.1 \mathrm{~ns}$, and residues 914-1348 come from the constant velocity SMD simulation S11d at 61.694 ns. Residues 360-369 in chain B of PCDH15 come from the constant velocity SMD 
$1 \quad 2.755 \mathrm{~ns}$, residues 496-597 come from the equilibration simulation S2a at $4.182 \mathrm{~ns}$, residues 598-800 come from constant velocity SMD simulation S2c at $8.1 \mathrm{~ns}$, residues 801-911 come from constant velocity SMD simulation S2c at 9.24 ns, residues 912-1021 come from constant velocity SMD simulation S11d at $34.384 \mathrm{ns,} \mathrm{and} \mathrm{residues}$ 1022-1348 come from constant velocity SMD simulation S11d at 61.694 ns. Coordinates for tetrameric complexes are available upon request.

7 All protein structures and models described above, along with crystallographic water molecules, had hydrogen atoms automatically added by psfgen. Residues D, E, K and R were assumed charged. Histidine residues were assumed neutral, and their protonation state was chosen to favor the formation of evident hydrogen bonds.

10 Systems were solvated in explicit water, neutralized, and ionized with randomly placed ions so as to mimic a 11 physiological endolymph environment with $150 \mathrm{mM} \mathrm{KCl}$. The $m m($ PCDH15 EC1-MAD12) $2+m m(\mathrm{CDH} 23$ 12 EC1-3 $)_{2}$ tetramer was solvated in two different water boxes, a smaller system for SMD and a larger system for 13 long free-dynamics equilibrations.

\section{MD simulations using NAMD}

MD simulations were performed using NAMD 2.11, 2.12, and $2.13^{127}$, the CHARMM36 force field for proteins with the CMAP correction and the TIP3P model for water ${ }^{128}$. A cutoff of $12 \AA$ (with a switching function starting at $10 \AA$ ) was used for van der Waals interactions. Periodic boundary conditions were used along with the Particle Mesh Ewald method to compute long-range electrostatic forces without cutoff and with a grid point density of $>1 \AA^{-3}$. A uniform 2 fs integration time step was used together with SHAKE. Langevin dynamics was utilized to enforce constant temperature $T=300 \mathrm{~K}$ with a damping coefficient of $0.1 \mathrm{ps}^{-1}$, unless otherwise stated. Constant pressure simulations $(N p T)$ at 1 atm were conducted using the hybrid Nosé-Hoover Langevin piston method with a 200 fs decay period and a 100 fs damping time constant.

\section{Simulations and analysis tools}

26 Each system was energy-minimized and equilibrated in the $N p T$ ensemble, and the resulting state was used to 27 perform subsequent equilibrium and SMD simulations (Table 3 and Table S8). Constant-velocity stretching 
1 simulations used the SMD method and the NAMD Tcl forces interface ${ }^{59-61,129}$, whereby C $\alpha$ atoms of specific

2 terminal residues were attached to independent virtual springs of stiffness $k_{s}=1 \mathrm{kcal} \mathrm{mol}^{-1} \AA^{-2}$. For SMD

3 simulations of dimeric (simulations S11b-d) and heterotetrameric systems (simulations S3b-d, S4b-d, S5b-d,

4 S6b-d, S7b-d, S8b-d, S9b-d), these springs were connected to virtual slabs attached to a third stretching spring

$5 \quad\left(k_{s}=1 \mathrm{kcal} \mathrm{mol}^{-1} \AA^{-2}\right)$. The free ends of the stretching springs were moved away from the protein in opposite

6 directions at a constant velocity. The stretching direction was set along the $x$-axis matching the vector connecting

7 terminal regions of the proteins in the corresponding simulated complexes. Applied forces were computed using

8 the extension of the virtual springs. Stiffness was computed through linear regression fits of force-distance plots.

9 Maximum force peaks and their averages were computed from 50 ps running averages used to eliminate local

10 fluctuations. The end-to-end distance in constant-velocity SMD simulations of heterotetrameric systems (S3 to

11 S10 in Table 3 and Table S8) was computed as the separation between the center-of-mass of the C $\alpha$ atoms stretched on one end and the center-of-mass of the $\mathrm{C} \alpha$ atoms stretched in the opposite direction on the other end of the system. Principal axes of EC repeats were computed using the Orient VMD plugin. Sequence alignments were performed with MUSCLE. Plots and curve fits were prepared with Xmgrace. Molecular images were created with the molecular graphics program $\mathrm{VMD}^{126}$. PCDH15 ectodomain sequences were compared among various species using data obtained from the NCBI protein database (Table S1). Ectodomain sequences equivalent to $h s$ PCDH15 CD1-1 (without exon 12a, unless indicated) were split into their respective EC repeats prior to alignment, which was done using the ClustalW algorithm ${ }^{130}$ within Geneious ${ }^{131}$ to determine the percent sequence identity for each EC repeat (Fig. S1 and S3A). Aligned sequences were loaded into JalView ${ }^{132}$ and colored based on sequence conservation with a $45 \%$ conservation threshold. Comparison of EC repeats within the human PCDH15 protein was carried out by aligning individual EC repeats to each other using ClustalW on

22 Geneious. The alignment (Fig. S2) was imported into the Sequence Identity and Similarity (SIAS) server ${ }^{133}$ to obtain the sequence identity matrix in Fig. S3B. Structural comparisons and computation of core RMSD were obtained using COOT $^{109}$.

\section{DATA AVAILABILITY}

27 Coordinates for all structures presented here have been deposited in the Protein Data Bank with entry codes 
G16D/N369D/Q370N + mm CDH23 EC1-2 T15E), 5ULY (hs PCDH15 EC2-3), 6EB5 (hs PCDH15 EC2-3

V250N), 6E8F ( $h \mathrm{~s}$ PCDH15 EC3-5 CD2-1), 5W1D (mm PCDH15 EC4-7), 6BXU (mm PCDH15 EC5-7 I582T),

6BWN ( $\mathrm{mm}$ PCDH15 EC6-7), 5TPK ( $\mathrm{mm}$ PCDH15 EC7-8 V875A), and 6EET ( $\mathrm{mm}$ PCDH15 EC9-MAD12).

Other raw data and data sets generated and/or analyzed during the current study are available from the corresponding author on reasonable request.

\section{REFERENCES}

1. Perozo, E. Gating prokaryotic mechanosensitive channels. Nat. Rev. Mol. Cell Biol. 7, 109-119 (2006).

2. Kung, C., Martinac, B. \& Sukharev, S. Mechanosensitive Channels in Microbes. Annu. Rev. Microbiol. 64, 313-329 (2010).

3. Haswell, E. S., Phillips, R. \& Rees, D. C. Mechanosensitive channels: what can they do and how do they do it? Structure 19, 1356-69 (2011).

4. Murthy, S. E., Dubin, A. E. \& Patapoutian, A. Piezos thrive under pressure: mechanically activated ion channels in health and disease. Nat. Rev. Mol. Cell Biol. 18, 771-783 (2017).

5. Fettiplace, R. \& Kim, K. X. The physiology of mechanoelectrical transduction channels in hearing. Physiol. Rev. 94, 951-986 (2014).

6. Hudspeth, A. J. Integrating the active process of hair cells with cochlear function. Nat. Rev. Neurosci. 15, 600-614 (2014).

7. Katta, S., Krieg, M. \& Goodman, M. B. Feeling Force: Physical and Physiological Principles Enabling Sensory Mechanotransduction. Annu. Rev. Cell Dev. Biol. 31, 347-371 (2015).

8. Corey, D. P., Ó Maoiléidigh, D. \& Ashmore, J. F. in 75-111 (Springer, Cham, 2017). doi:10.1007/978-3-319-52073-5 4

9. Pickles, J. O., Comis, S. D. \& Osborne, M. P. Cross-links between stereocilia in the guinea pig organ of Corti, and their possible relation to sensory transduction. Hear. Res. 15, 103-112 (1984).

10. Assad, J. a, Shepherd, G. M. \& Corey, D. P. Tip-link integrity and mechanical transduction in vertebrate hair cells. Neuron 7, 985-994 (1991).

11. Kachar, B., Parakkal, M., Kurc, M., Zhao, Y. -d. \& Gillespie, P. G. High-resolution structure of hair-cell tip links. Proc. Natl. Acad. Sci. 97, 13336-13341 (2000).

12. Basu, A., Lagier, S., Vologodskaia, M., Fabella, B. A. \& Hudspeth, A. J. Direct mechanical stimulation of tip links in hair cells through DNA tethers. Elife 5, (2016).

13. Furness, D. N., Katori, Y., Nirmal Kumar, B. \& Hackney, C. M. The dimensions and structural attachments of tip links in mammalian cochlear hair cells and the effects of exposure to different levels of extracellular calcium. Neuroscience 154, 10-21 (2008).

14. Hackney, C. M. et al. The composition and role of cross links in mechanoelectrical transduction in vertebrate sensory hair cells. J. Cell Sci. 126, 1721-31 (2013).

15. Pepermans, E. \& Petit, C. The tip-link molecular complex of the auditory mechano-electrical transduction machinery. Hear. Res. 330, 10-7 (2015).

16. Jaiganesh, A., Narui, Y., Araya-Secchi, R. \& Sotomayor, M. Beyond Cell-Cell Adhesion: Sensational Cadherins for Hearing and Balance. Cold Spring Harb. Perspect. Biol. a029280 
(2017). doi:10.1101/cshperspect.a029280

17. Zhao, Y., Yamoah, E. N. \& Gillespie, P. G. Regeneration of broken tip links and restoration of mechanical transduction in hair cells. Proc. Natl. Acad. Sci. U. S. A. 93, 15469-15474 (1996).

18. Siemens, J. et al. Cadherin 23 is a component of the tip link in hair-cell stereocilia. Nature 428, 950-955 (2004).

19. Söllner, C. et al. Mutations in cadherin 23 affect tip links in zebrafish sensory hair cells. Nature 428, 955-959 (2004).

20. Ahmed, Z. M. et al. The tip-link antigen, a protein associated with the transduction complex of sensory hair cells, is protocadherin-15. J. Neurosci. 26, 7022-7034 (2006).

21. Kazmierczak, P. et al. Cadherin 23 and protocadherin 15 interact to form tip-link filaments in sensory hair cells. Nature 449, 87-91 (2007).

22. Di Palma, F. et al. Mutations in Cdh23, encoding a new type of cadherin, cause stereocilia disorganization in waltzer, the mouse model for Usher syndrome type 1D. Nat. Genet. 27, 103-7 (2001).

23. Alagramam, K. N. et al. Mutations in the novel protocadherin PCDH15 cause Usher syndrome type 1F. Hum. Mol. Genet. 10, 1709-1718 (2001).

24. Friedman, T. B., Schultz, J. M., Ahmed, Z. M., Tsilou, E. T. \& Brewer, C. C. in Medical Genetics in the Clinical Practice of ORL 70, 56-65 (KARGER, 2011).

25. Apostolopoulou, M. \& Ligon, L. Cadherin-23 mediates heterotypic cell-cell adhesion between breast cancer epithelial cells and fibroblasts. PLoS One 7, e33289 (2012).

26. Rouget-Quermalet, V. et al. Protocadherin 15 (PCDH15): a new secreted isoform and a potential marker for NK/T cell lymphomas. Oncogene 25, 2807-2811 (2006).

27. Zhang, Q. et al. Germline Mutations in CDH23, Encoding Cadherin-Related 23, Are Associated with Both Familial and Sporadic Pituitary Adenomas. Am. J. Hum. Genet. 100, 817-823 (2017).

28. Vanniya. S, P., Srisailapathy, C. R. S. \& Kunka Mohanram, R. The tip link protein Cadherin-23: From Hearing Loss to Cancer. Pharmacol. Res. 130, 25-35 (2018).

29. Sannigrahi, M. K., Srinivas, C. S., Deokate, N. \& Rakshit, S. The strong propensity of Cadherin23 for aggregation inhibits cell migration. Mol. Oncol. 1878-0261.12469 (2019). doi:10.1002/1878-0261.12469

30. Rzadzinska, A. K. \& Steel, K. P. Presence of interstereocilial links in waltzer mutants suggests Cdh23 is not essential for tip Link formation. Neuroscience 158, 365-368 (2009).

31. Indzhykulian, A. A. et al. Molecular Remodeling of Tip Links Underlies Mechanosensory Regeneration in Auditory Hair Cells. PLoS Biol. 11, e1001583 (2013).

32. Lelli, A., Kazmierczak, P., Kawashima, Y., Müller, U. \& Holt, J. R. Development and regeneration of sensory transduction in auditory hair cells requires functional interaction between cadherin-23 and protocadherin-15. J. Neurosci. 30, 11259-69 (2010).

33. Sotomayor, M., Weihofen, W. A., Gaudet, R. \& Corey, D. P. Structure of a force-conveying cadherin bond essential for inner-ear mechanotransduction. Nature 492, 128-32 (2012).

34. Geng, R. et al. Noddy, a mouse harboring a missense mutation in protocadherin-15, reveals the impact of disrupting a critical interaction site between tip-link cadherins in inner ear hair cells. $J$. Neurosci. 33, 4395-404 (2013).

35. Abdi, S. et al. Diversity of the Genes Implicated in Algerian Patients Affected by Usher Syndrome. PLoS One 11, e0161893 (2016).

36. Choudhary, D., Kumar, A., Magliery, T. J. \& Sotomayor, M. Using thermal scanning assays to test protein-protein interactions of inner-ear cadherins. PLoS One 12, e0189546 (2017).

37. Narui, Y. \& Sotomayor, M. Tuning Inner-Ear Tip-Link Affinity Through Alternatively Spliced Variants of Protocadherin-15. Biochemistry 57, acs.biochem.7b01075 (2018).

38. Dionne, G. et al. Mechanotransduction by PCDH15 Relies on a Novel cis-Dimeric Architecture. Neuron 99, 480-492.e5 (2018).

39. Ge, J. et al. Structure of mouse protocadherin 15 of the stereocilia tip link in complex with 
LHFPL5. Elife 7, (2018).

40. De-la-Torre, P., Choudhary, D., Araya-Secchi, R., Narui, Y. \& Sotomayor, M. A Mechanically Weak Extracellular Membrane-Adjacent Domain Induces Dimerization of Protocadherin-15. Biophys. J. 115, 2368-2385 (2018).

41. Elledge, H. M. et al. Structure of the $\mathrm{N}$ terminus of cadherin 23 reveals a new adhesion mechanism for a subset of cadherin superfamily members. Proc. Natl. Acad. Sci. U. S. A. 107, 10708-10712 (2010).

42. Sotomayor, M., Weihofen, W. A., Gaudet, R. \& Corey, D. P. Structural Determinants of Cadherin-23 Function in Hearing and Deafness. Neuron 66, 85-100 (2010).

43. Araya-Secchi, R., Neel, B. L. \& Sotomayor, M. An elastic element in the protocadherin-15 tip link of the inner ear. Nat. Commun. 7, 13458 (2016).

44. Powers, R. E., Gaudet, R. \& Sotomayor, M. A Partial Calcium-Free Linker Confers Flexibility to Inner-Ear Protocadherin-15. Structure 25, 482-495 (2017).

45. Jaiganesh, A. et al. Zooming in on Cadherin-23: Structural Diversity and Potential Mechanisms of Inherited Deafness. Structure (2018). doi:10.1016/j.str.2018.06.003

46. Corey, D. P. \& Hudspeth, A. J. Kinetics of the receptor current in bullfrog saccular hair cells. J. Neurosci 3, 962-976 (1983).

47. Howard, J. \& Hudspeth, A. J. Compliance of the hair bundle associated with gating of mechanoelectrical transduction channels in the bullfrog's saccular hair cell. Neuron 1, 189-199 (1988).

48. Cheung, E. L. M. \& Corey, D. P. Ca2+ Changes the Force Sensitivity of the Hair-Cell Transduction Channel. Biophys. J. 90, 124-139 (2006).

49. Pickles, J. O. \& Corey, D. P. Mechanoelectrical transduction by hair cells. Trends Neurosci. 15, 254-9 (1992).

50. Jin, X. et al. Crystal structures of Drosophila N-cadherin ectodomain regions reveal a widely used class of $\mathrm{Ca}^{2}+$-free interdomain linkers. Proc. Natl. Acad. Sci. U. S. A. 109, E127-34 (2012).

51. Tsukasaki, Y. et al. Giant cadherins Fat and Dachsous self-bend to organize properly spaced intercellular junctions. Proc. Natl. Acad. Sci. 111, 16011-16016 (2014).

52. Bosher, S. K. \& Warren, R. L. Very low calcium content of cochlear endolymph, an extracellular fluid. Nature 273, 377-378 (1978).

53. Salt, A. N., Inamura, N., Thalmann, R. \& Vora, A. Calcium gradients in inner ear endolymph. Am. J. Otolaryngol. 10, 371-5 (1989).

54. Yamoah, E. N. et al. Plasma membrane Ca2+-ATPase extrudes Ca2+ from hair cell stereocilia. J. Neurosci. 18, 610-24 (1998).

55. Strimbu, C. E., Prasad, S., Hakizimana, P. \& Fridberger, A. Control of hearing sensitivity by tectorial membrane calcium. Proc. Natl. Acad. Sci. 201805223 (2019).

doi:10.1073/pnas. 1805223116

56. Mammano, F., Bortolozzi, M., Ortolano, S. \& Anselmi, F. Ca2+ Signaling in the Inner Ear. Physiology 22, 131-144 (2007).

57. Pei, J. \& Grishin, N. V. Expansion of divergent SEA domains in cell surface proteins and nucleoporin 54. Protein Sci. 26, 617-630 (2017).

58. Isralewitz, B., Gao, M. \& Schulten, K. Steered molecular dynamics and mechanical functions of proteins. Curr. Opin. Struct. Biol. 11, 224-230 (2001).

59. Grubmüller, H. Force probe molecular dynamics simulations. Methods Mol. Biol. 305, 493-515 (2005).

60. Gräter, F., Shen, J., Jiang, H., Gautel, M. \& Grubmüller, H. Mechanically induced titin kinase activation studied by force-probe molecular dynamics simulations. Biophys. J. 88, 790-804 (2005).

61. Sotomayor, M. \& Schulten, K. Single-molecule experiments in vitro and in silico. Science 316, 1144-1148 (2007). 
62. Overduin, M. et al. Solution structure of the epithelial cadherin domain responsible for selective cell adhesion. Science 267, 386-9 (1995).

63. Shapiro, L. et al. Structural basis of cell-cell adhesion by cadherins. Nature 374, 327-37 (1995).

64. Nagar, B., Overduin, M., Ikura, M. \& Rini, J. M. Structural basis of calcium-induced E-cadherin rigidification and dimerization. Nature 380, 360-4 (1996).

65. Goodman, K. M. et al. Structural Basis of Diverse Homophilic Recognition by Clustered $\alpha$ - and $\beta$-Protocadherins. Neuron (2016). doi:10.1016/j.neuron.2016.04.004

66. Goodman, K. M. et al. $\gamma$-Protocadherin structural diversity and functional implications. Elife 5, (2016).

67. Nicoludis, J. M. et al. Structure and Sequence Analyses of Clustered Protocadherins Reveal Antiparallel Interactions that Mediate Homophilic Specificity. Structure 23, 2087-98 (2015).

68. Nicoludis, J. M. et al. Antiparallel protocadherin homodimers use distinct affinity- and specificity-mediating regions in cadherin repeats 1-4. Elife 5, (2016).

69. Cooper, S. R., Jontes, J. D. \& Sotomayor, M. Structural determinants of adhesion by Protocadherin-19 and implications for its role in epilepsy. Elife 5, (2016).

70. Li, Y. et al. Mechanism of E-cadherin dimerization probed by NMR relaxation dispersion. Proc. Natl. Acad. Sci. 110, 16462-16467 (2013).

71. Ahmed, Z. M. et al. PCDH15 is expressed in the neurosensory epithelium of the eye and ear and mutant alleles are responsible for both USH1F and DFNB23. Hum. Mol. Genet. 12, 3215-23 (2003).

72. Aurora, R. \& Rosee, G. D. Helix capping. Protein Sci. 7, 21-38 (1998).

73. Webb, S. W. et al. Regulation of PCDH15 function in mechanosensory hair cells by alternative splicing of the cytoplasmic domain. Development 138, 1607-17 (2011).

74. Pepermans, E. et al. The CD2 isoform of protocadherin-15 is an essential component of the tiplink complex in mature auditory hair cells. EMBO Mol. Med. 6, 984-992 (2014).

75. Panjkovich, A. \& Svergun, D. I. Deciphering conformational transitions of proteins by small angle X-ray scattering and normal mode analysis. Phys. Chem. Chem. Phys. 18, 5707-19 (2016).

76. Goodyear, R. J., Forge, A., Legan, P. K. \& Richardson, G. P. Asymmetric distribution of cadherin 23 and protocadherin 15 in the kinocilial links of avian sensory hair cells. J. Comp. Neurol. 518, 4288-4297 (2010).

77. Richardson, G. P. \& Petit, C. Hair-Bundle Links: Genetics as the Gateway to Function. Cold Spring Harb. Perspect. Med. a033142 (2019). doi:10.1101/cshperspect.a033142

78. Jaramillo, F. \& Hudspeth, A. J. Displacement-clamp measurement of the forces exerted by gating springs in the hair bundle. Proc. Natl. Acad. Sci. U. S. A. 90, 1330-4 (1993).

79. Tobin, M., Chaiyasitdhi, A., Michel, V., Michalski, N. \& Martin, P. Stiffness and tension gradients of the hair cell's tip-link complex in the mammalian cochlea. Elife 8, (2019).

80. Kulahin, N. et al. Structural Model and trans-Interaction of the Entire Ectodomain of the Olfactory Cell Adhesion Molecule. Structure 19, 203-211 (2011).

81. Robles, L. \& Ruggero, M. A. Mechanics of the mammalian cochlea. Physiol. Rev. 81, 13051352 (2001).

82. Lee, E. H., Hsin, J., Sotomayor, M., Comellas, G. \& Schulten, K. Discovery through the computational microscope. Structure 17, 1295-306 (2009).

83. Rico, F., Gonzalez, L., Casuso, I., Puig-Vidal, M. \& Scheuring, S. High-speed force spectroscopy unfolds titin at the velocity of molecular dynamics simulations. Science 342, 741743 (2013).

84. Cailliez, F. \& Lavery, R. Cadherin mechanics and complexation: the importance of calcium binding. Biophys. J. 89, 3895-3903 (2005).

85. Sotomayor, M. \& Schulten, K. The allosteric role of the $\mathrm{Ca} 2+$ switch in adhesion and elasticity of C-cadherin. Biophys. J. 94, 4621-4633 (2008).

86. Manibog, K., Li, H., Rakshit, S. \& Sivasankar, S. Resolving the molecular mechanism of 
cadherin catch bond formation. Nat. Commun. 5, 3941 (2014).

87. Pokutta, S., Herrenknecht, K., Kemler, R. \& Engel, J. Conformational changes of the recombinant extracellular domain of E-cadherin upon calcium binding. Eur. J. Biochem. 223, 1019-1026 (1994).

88. Rakshit, S. \& Sivasankar, S. Biomechanics of cell adhesion: how force regulates the lifetime of adhesive bonds at the single molecule level. Phys. Chem. Chem. Phys. 16, 2211 (2014).

89. Bartsch, T. F. et al. Elasticity of individual protocadherin 15 molecules implicates tip links as the gating springs for hearing. Proc. Natl. Acad. Sci. 116, 11048-11056 (2019).

90. Sotomayor, M., Corey, D. P. \& Schulten, K. In search of the hair-cell gating spring: Elastic properties of ankyrin and cadherin repeats. Structure 13, 669-682 (2005).

91. Corey, D. P. \& Sotomayor, M. Tightrope act. Nature 428, (2004).

92. Bartsch, T. F. \& Hudspeth, A. J. A New Twist on Tip Links. Neuron 99, 423-425 (2018).

93. Ruggero, M. A., Rich, N. C., Recio, A., Narayan, S. S. \& Robles, L. Basilar-membrane responses to tones at the base of the chinchilla cochlea. J. Acoust. Soc. Am. 101, 2151-63 (1997).

94. Lee, H. Y. et al. Two-Dimensional Cochlear Micromechanics Measured In Vivo Demonstrate Radial Tuning within the Mouse Organ of Corti. J. Neurosci. 36, 8160-73 (2016).

95. Fridberger, A., Tomo, I., Ulfendahl, M. \& Boutet de Monvel, J. Imaging hair cell transduction at the speed of sound: Dynamic behavior of mammalian stereocilia. Proc. Natl. Acad. Sci. 103, 1918-1923 (2006).

96. Wang, Y., Puria, S., Steele, C. R. \& Ricci, A. J. Inner hair cell stereocilia movements captured in-situ by a high-speed camera with subpixel image processing. in AIP Conference Proceedings 1965, 120004 ( AIP Publishing LLC , 2018).

97. Bell, G. I. Models for the specific adhesion of cells to cells. Science 200, 618-27 (1978).

98. Evans, E. \& Ritchie, K. Dynamic strength of molecular adhesion bonds. Biophys. J. 72, 15411555 (1997).

99. Evans, E. A. \& Calderwood, D. A. Forces and bond dynamics in cell adhesion. Science 316, 1148-53 (2007).

100. Dudko, O. K. Decoding the mechanical fingerprints of biomolecules. Q. Rev. Biophys. 1-14 (2015). doi:10.1017/S0033583515000220

101. Izrailev, S., Stepaniants, S., Balsera, M., Oono, Y. \& Schulten, K. Molecular dynamics study of unbinding of the avidin-biotin complex. Biophys. J. 72, 1568-1581 (1997).

102. Sheridan, S., Gräter, F. \& Daday, C. How Fast Is Too Fast in Force-Probe Molecular Dynamics Simulations? J. Phys. Chem. B 123, 3658-3664 (2019).

103. Eatock, R. A. Adaptation in hair cells. Annu. Rev. Neurosci. 23, 285-314 (2000).

104. Libé-Philippot, B. et al. Auditory cortex interneuron development requires cadherins operating hair-cell mechanoelectrical transduction. Proc. Natl. Acad. Sci. U. S. A. 114, 7765-7774 (2017).

105. Sahly, I. et al. Localization of Usher 1 proteins to the photoreceptor calyceal processes, which are absent from mice. J. Cell Biol. 199, 381-399 (2012).

106. Alagramam, K. N. et al. The mouse Ames waltzer hearing-loss mutant is caused by mutation of Pcdh15, a novel protocadherin gene. Nat. Genet. 27, 99-102 (2001).

107. Otwinowski, Z. \& Minor, W. Processing of X-ray diffraction data. Methods Enzym. 276, 307326 (1997).

108. McCoy, A. J. et al. Phaser crystallographic software. J. Appl. Crystallogr. 40, 658-674 (2007).

109. Emsley, P., Lohkamp, B., Scott, W. G. \& Cowtan, K. Features and development of Coot. Acta Crystallogr. D. Biol. Crystallogr. 66, 486-501 (2010).

110. Murshudov, G. N. et al. REFMAC5 for the refinement of macromolecular crystal structures. Acta Crystallogr. Sect. D Biol. Crystallogr. 67, 355-367 (2011).

111. Laskowski, R. A., MacArthur, M. W., Moss, D. S., Thornton, J. M. \& IUCr. PROCHECK: a program to check the stereochemical quality of protein structures. J. Appl. Crystallogr. 26, 283- 
291 (1993).

112. Hooft, R. W. W., Vriend, G., Sander, C. \& Abola, E. E. Errors in protein structures. Nature 381, 272-272 (1996).

113. Zheng, H. et al. Validation of metal-binding sites in macromolecular structures with the CheckMyMetal web server. Nat. Protoc. 9, 156-170 (2014).

114. Dyer, K. N. et al. High-throughput SAXS for the characterization of biomolecules in solution: a practical approach. Methods Mol. Biol. 1091, 245-258 (2014).

115. Classen, S. et al. Implementation and performance of SIBYLS: a dual endstation small-angle Xray scattering and macromolecular crystallography beamline at the Advanced Light Source. $J$. Appl. Crystallogr. 46, 1-13 (2013).

116. Rambo, R. P. \& Tainer, J. A. Characterizing flexible and intrinsically unstructured biological macromolecules by SAS using the Porod-Debye law. Biopolymers 95, 559-571 (2011).

117. Konarev, P. V, Volkov, V. V, Sokolova, A. V, Koch, M. H. J. \& Svergun, D. I. PRIMUS: A Windows PC-based system for small-angle scattering data analysis. J. Appl. Crystallogr. 36, 1277-1282 (2003).

118. Franke, D. et al. ATSAS $2.8:$ a comprehensive data analysis suite for small-angle scattering from macromolecular solutions. J. Appl. Crystallogr. 50, 1212-1225 (2017).

119. Svergun, D. I. Determination of the Regularization Parameter in Indirect- Transform Methods Using Perceptual Criteria. J. Appl. Crystallogr. 25, 495-503 (1992).

120. Fischer, H., de Oliveira Neto, M., Napolitano, H. B., Polikarpov, I. \& Craievich, A. F. Determination of the molecular weight of proteins in solution from a single small-angle X-ray scattering measurement on a relative scale. J. Appl. Crystallogr. 43, 101-109 (2010).

121. Schneidman-Duhovny, D., Hammel, M., Tainer, J. A. \& Sali, A. Accurate SAXS profile computation and its assessment by contrast variation experiments. Biophys. J. 105, 962-974 (2013).

122. Dam, J., Velikovsky, C. A., Mariuzza, R. A., Urbanke, C. \& Schuck, P. Sedimentation velocity analysis of heterogeneous protein-protein interactions: Lamm equation modeling and sedimentation coefficient distributions c(s). Biophys. J. 89, 619-34 (2005).

123. Schuck, P. Size-Distribution Analysis of Macromolecules by Sedimentation Velocity Ultracentrifugation and Lamm Equation Modeling. Biophys. J. 78, 1606-1619 (2000).

124. Zhao, H., Brautigam, C. A., Ghirlando, R. \& Schuck, P. Overview of Current Methods in Sedimentation Velocity and Sedimentation Equilibrium Analytical Ultracentrifugation. Curr. Protoc. Protein Sci. 71, 20.12.1-20.12.49 (2013).

125. Brautigam, C. A. in Methods in enzymology 562, 109-133 (2015).

126. Humphrey, W., Dalke, A. \& Schulten, K. VMD: Visual molecular dynamics. J. Mol. Graph. 14, 33-38 (1996).

127. Phillips, J. C. et al. Scalable molecular dynamics with NAMD. J. Comput. Chem. 26, 1781$1802(2005)$.

128. Huang, J. \& MacKerell, A. D. J. CHARMM36 all-atom additive protein force field: Validation based on comparison to NMR data. J. Comput. Chem. 34, 2135-2145 (2014).

129. Isralewitz, B., Baudry, J., Gullingsrud, J., Kosztin, D. \& Schulten, K. Steered Molecular Dynamics Investigations of Protein Function. J. Mol. Graph. Model. 19, 13-25 (2001).

130. Larkin, M. A. et al. Clustal W and Clustal X version 2.0. Bioinformatics 23, 2947-2948 (2007).

131. Kearse, M. et al. Geneious Basic: an integrated and extendable desktop software platform for the organization and analysis of sequence data. Bioinformatics 28, 1647-9 (2012).

132. Waterhouse, A. M., Procter, J. B., Martin, D. M. A., Clamp, M. \& Barton, G. J. Jalview Version 2--a multiple sequence alignment editor and analysis workbench. Bioinformatics 25, 1189-1191 (2009).

133. Garcia-Boronat, M., Diez-Rivero, C. M., Reinherz, E. L. \& Reche, P. A. PVS: a web server for protein sequence variability analysis tuned to facilitate conserved epitope discovery. Nucleic 
Acids Res. 36, W35-W41 (2008).

134. Grossman, S. R. et al. A composite of multiple signals distinguishes causal variants in regions of positive selection. Science (80-. ). 327, 883-886 (2010).

135. Saleha, S., Ajmal, M., Jamil, M., Nasir, M. \& Hameed, A. In silico analysis of a disease-causing mutation in PCDH15 gene in a consanguineous Pakistani family with Usher phenotype. Int. J. Ophthalmol. 9, 662-8 (2016).

136. Zheng, Q. Y. et al. A new spontaneous mutation in the mouse protocadherin 15 gene. Hear. Res. 219, 110-120 (2006).

137. Alagramam, K. N. et al. Mutations in Protocadherin 15 and Cadherin 23 Affect Tip Links and Mechanotransduction in Mammalian Sensory Hair Cells. PLoS One 6, e19183 (2011).

138. Zhan, Y., Liu, M., Chen, D., Chen, K. \& Jiang, H. Novel mutation located in EC7 domain of protocadherin-15 uncovered by targeted massively parallel sequencing in a family segregating non-syndromic deafness DFNB23. Int. J. Pediatr. Otorhinolaryngol. 79, 983-6 (2015).

139. Ahmed, Z. M. et al. Gene structure and mutant alleles of PCDH15: nonsyndromic deafness DFNB23 and type 1 Usher syndrome. Hum. Genet. 124, 215-223 (2008).

140. Chen, D. et al. Mutation in PCDH15 may modify the phenotypic expression of the 7511T $>$ C mutation in MT-TS1 in a Chinese Han family with maternally inherited nonsyndromic hearing loss. Int. J. Pediatr. Otorhinolaryngol. 79, 1654-1657 (2015).

141. Yang, T., Wei, X., Chai, Y., Li, L. \& Wu, H. Genetic etiology study of the non-syndromic deafness in Chinese Hans by targeted next-generation sequencing. Orphanet J. Rare Dis. 8, 85 (2013).

142. Doucette, L. et al. Profound, prelingual nonsyndromic deafness maps to chromosome 10q21 and is caused by a novel missense mutation in the Usher syndrome type IF gene PCDH15. Eur. J. Hum. Genet. 17, 554-64 (2009).

143. Miyagawa, M., Naito, T., Nishio, S., Kamatani, N. \& Usami, S. Targeted exon sequencing successfully discovers rare causative genes and clarifies the molecular epidemiology of Japanese deafness patients. PLoS One 8, e71381 (2013).

144. Schrauwen, I. et al. Novel digenic inheritance of PCDH15 and USH1G underlies profound nonsyndromic hearing impairment. BMC Med. Genet. 19, 122 (2018). 


\section{ACKNOWLEDGEMENTS}

2 We thank Michael Hammel and Daniel Rosenberg for data collection and initial SEC-SAXS data analysis,

3 Marina Bakhtina for assistance with AUC experiments, Benjamin Donovan for assistance with cloning, Michelle

4 E. Gray for maintenance of the Expi293 cell line, as well as members of the Sotomayor research group for their

5 feedback, training, and discussions. This work was supported by the Ohio State University, by the National

6 Institutes of Health - National Institute on Deafness and Other Communication Disorders (NIH/NIDCD R01

7 DC015271), and by the National Science Foundation through XSEDE (XRAC MCB140226). Simulations were

8 performed at the TACC-Stampede, PSC-Bridges, and OSC-Owens (PAS1037) supercomputers. Use of APS NE-

9 CAT beamlines was supported by NIH (P30 GM1241653 and S10 RR029205) and the Department of Energy

10 (DE-AC02-06CH11357) through grants GUP 40277, 49774, and 59521. SAXS data collection at the Advanced

11 Light Source SIBYLS beamline was funded through the DOE BER Integrated Diffraction Analysis

12 Technologies program and NIH grants NIGMS P30 GM124169 ALS-ENABLE and S10OD018483. B.L.N. was

13 supported by a 2017 Cellular, Molecular, and Biochemical Sciences Program training grant fellowship. P.D. and

14 R.A.-S. were Pelotonia fellows. L.N.W. received summer support from The Ohio State University Mayer 15 scholarship.

\section{AUTHOR CONTRIBUTIONS}

D.C. did cloning, protein expression, purification, crystallization, and X-ray crystal structure solution and refinement of $h s$ PCDH15 EC1-3 G16D/N369D/Q370N, hs PCDH15 EC1-3 G16D/N369D/Q370N + CDH23 EC1-2 T15E (assisted by P.D.), hs PCDH15 EC2-3 V250N, and hs PCDH15 EC3-5 CD2-1 (assisted by E.T.). D.C. designed and did initial tests with all mutations targeting the PCDH15 X-dimer, and performed all AUC experiments and analyses. D.C. and Y.N. did MALS experiments (assisted by P.D. and E.T.). Y.N. did cloning, protein expression, purification, crystallization and X-ray crystal structure solution and refinement of $\mathrm{mm}$ PCDH15 EC1-2BAP and $m m$ PCDH15 EC9-MAD12 (mammalian expression). Y.N. did cloning and initial protein expression of PCDH15's full-length ectodomain, which was further produced by D.C. and E.T. for 
1 cloning, protein expression, purification, and crystallization of $m m$ PCDH15 EC6-7. B.L.N. solved and refined

2 the $m m$ PCDH15 EC6-7 X-ray crystal structure. L.N.W. and M.S. did cloning, protein expression, purification,

3 crystallization, and X-ray crystal structure solution and refinement of $h s$ PCDH15 EC2-3. C.K. and M.S. did

4 cloning, protein expression, purification, crystallization, and X-ray crystal structure solution for $m m$ PCDH15

5 EC4-7. B.L.N., C.K., and M.S. refined the $m m$ PCDH15 EC4-7 X-ray crystal structure. C.C. and M.S. did

6 cloning, protein expression, purification, crystallization, and X-ray crystal structure solution and refinement of

$7 \mathrm{~mm}$ PCDH15 EC7-8 V875A. Cloning of $\mathrm{mm}$ PCDH15 EC9-MAD12 for bacterial expression was done by R.A.-

8 S. P.D. and D.C. prepared samples for SAXS experiments, which were analyzed by R.A.-S. Models of multi-

9 structure PCDH15 ectodomains were assembled by B.L.N. and M.S. Simulations were designed, carried out, and

10 analyzed by D.C., Y.N., B.L.N., and M.S. Manuscript and figures were prepared by D.C., Y.N., B.L.N., P.D.,

11 R.A-S., and M.S. with feedback from all co-authors. B.L.N. prepared all movies. M.S. trained, supervised, and

12 assisted co-authors in experimental design, cloning, crystal fishing/cryo-cooling, structure refinement and 13 deposition, and data analysis.

\section{COMPETING FINANCIAL INTERESTS}

16 M.S. is co-inventor on a patent application that describes the use of modified PCDH15 proteins for gene therapy.

17 The authors declare no other competing financial interests. 
FIGURES AND FIGURE LEGENDS
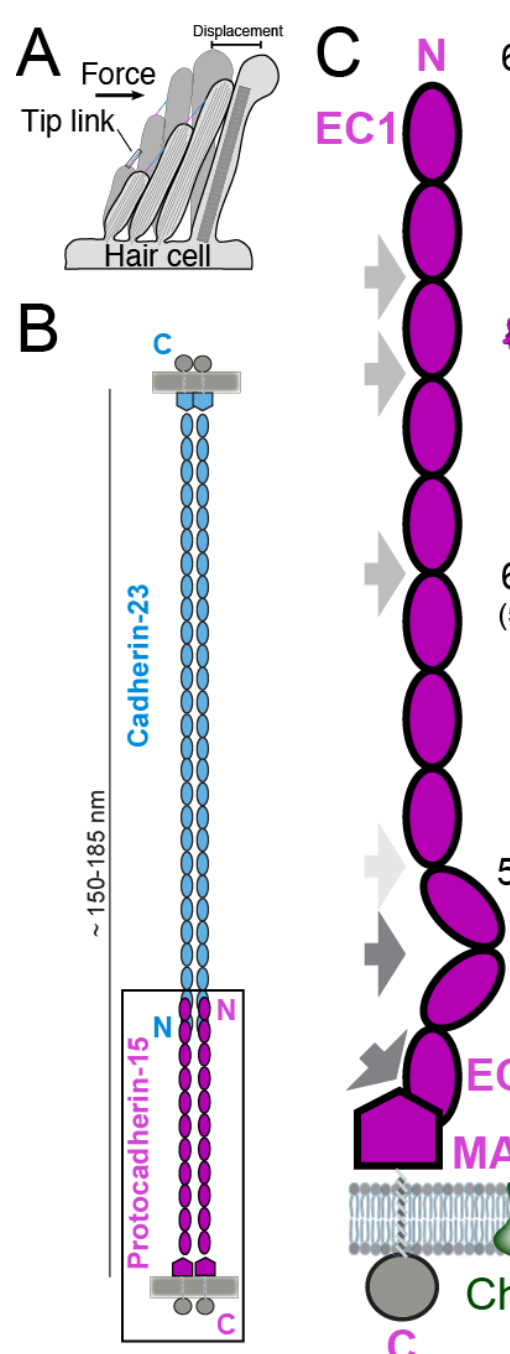

6N22 6MFO
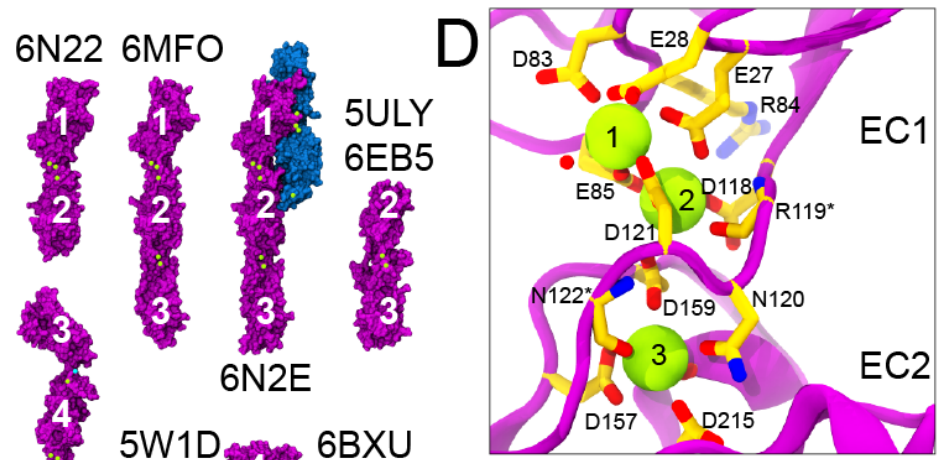

$5 \mathrm{~W} 1 \mathrm{D}, 6 \mathrm{BXU}$

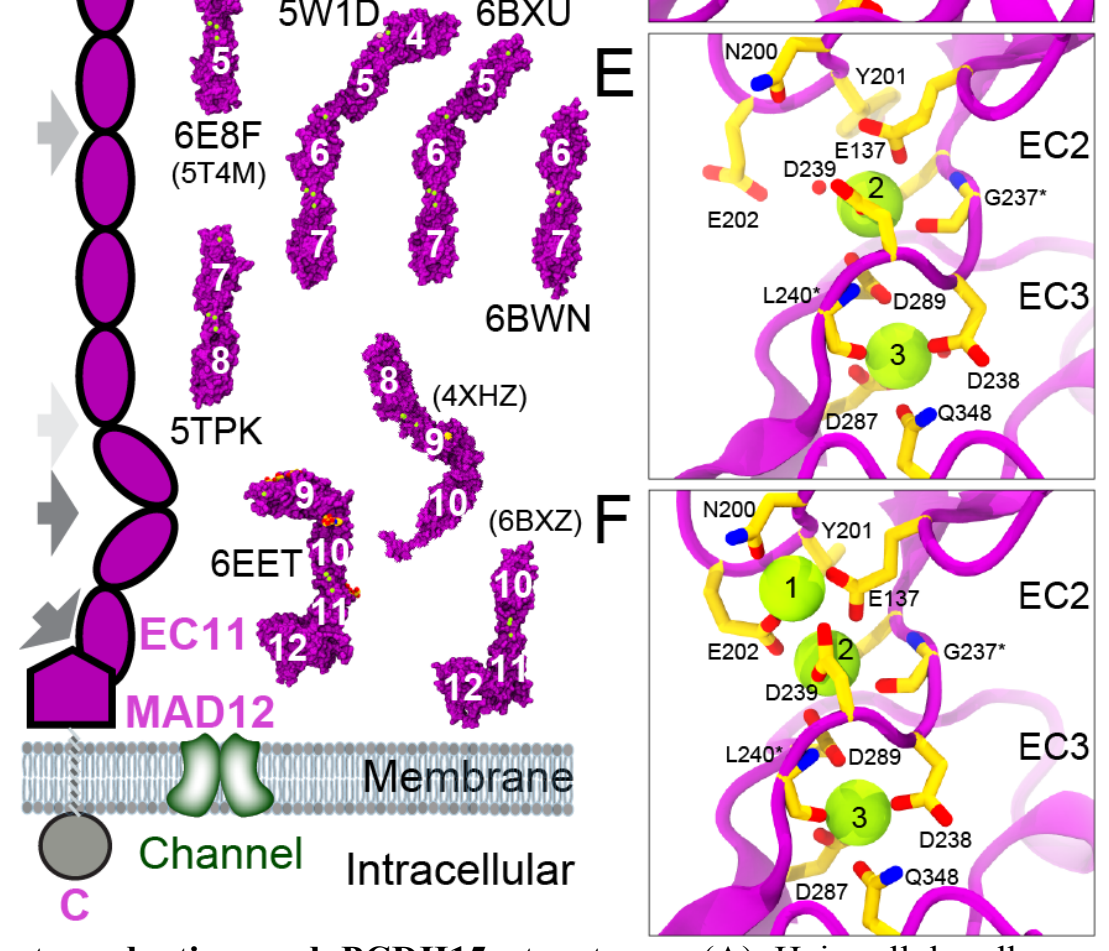

Figure 1. Inner-ear mechanotransduction and PCDH15 structures. (A) Hair-cell bundle row showing location of tip links. Force from sound displaces the bundle to activate the transduction apparatus. (B) The tip link is made of CDH23 (blue) and PCDH15 (purple). (C) Schematic representation of PCDH15 (left panel) and structures of PCDH15 fragments (right) used to build models of the entire PCDH15 ectodomain. PDB codes are indicated for all 11 structures presented here, with codes in parenthesis for three additional structures presented elsewhere ${ }^{40,43,44}$. All structures are in surface representation, with PCDH15 fragments in purple and CDH23 EC1-2 in blue. Gray arrows in the schematics indicate EC linkers with atypical canonical-like linker regions (EC8-9 in light gray), with partial $\mathrm{Ca}^{2+}$-free linker regions (EC2-3, EC3-4, and EC5-6 in gray), and with $\mathrm{Ca}^{2+}$ free linker regions (EC9-10 and EC11-MAD12 in dark gray). (D) Detail of a canonical $\mathrm{Ca}^{2+}$-binding linker region from $\mathrm{mm}$ PCDH15 EC1-2BAP. Protein backbone is shown in magenta ribbon. Relevant residues are shown in stick representation and labeled. Asterisk $\left(^{*}\right)$ denotes backbone coordination of $\mathrm{Ca}^{2+}$. Water molecules are shown as red spheres. Some backbone atoms and some water molecules are omitted for clarity. (E) Detail of a partial $\mathrm{Ca}^{2+}$-free linker region in $h s$ PCDH15 EC2-3 as in D. (F) The monomeric mutant $h s$ PCDH15 EC2-3 V250N binds all three $\mathrm{Ca}^{2+}$ ions. Residue p.E202, previously interacting with p.R254 (not shown), flips in to coordinate $\mathrm{Ca}^{2+}$ at sites 1 and 2 . 


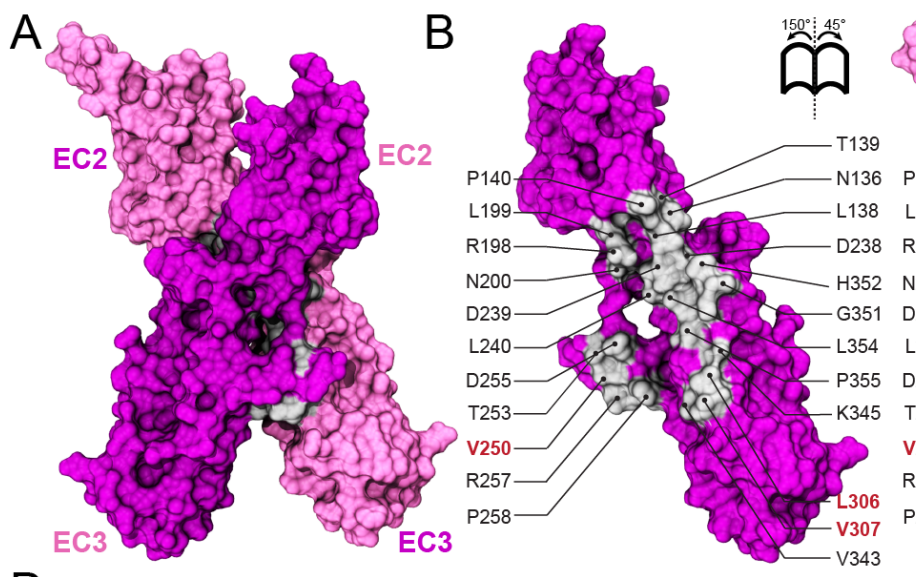

$\mathrm{D}$

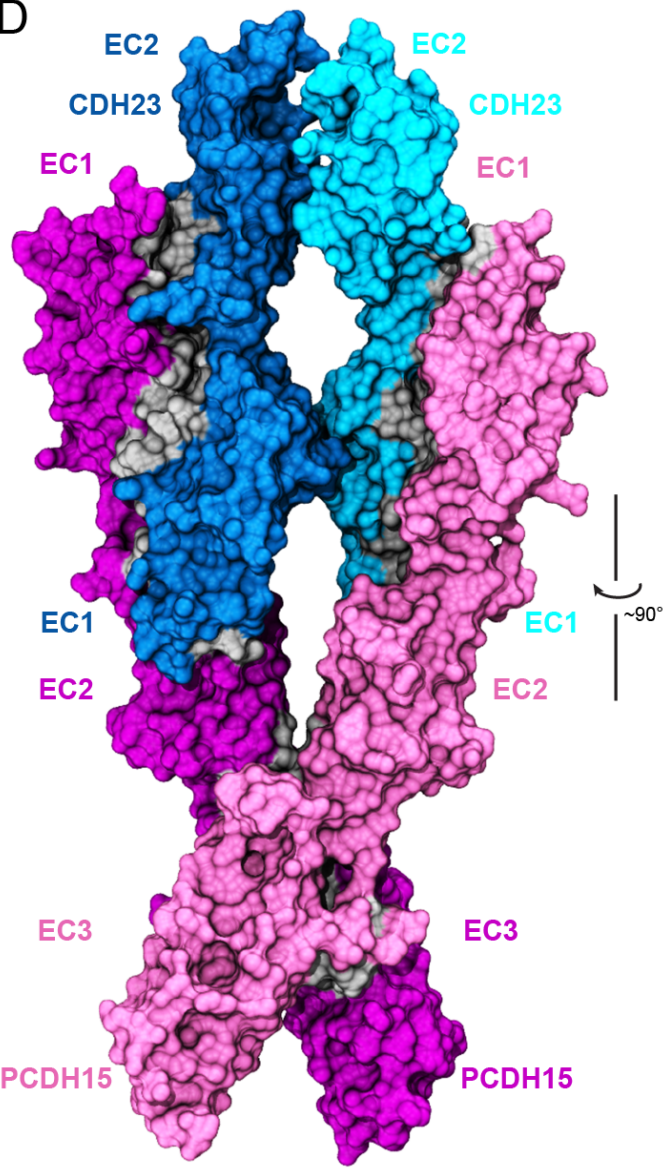

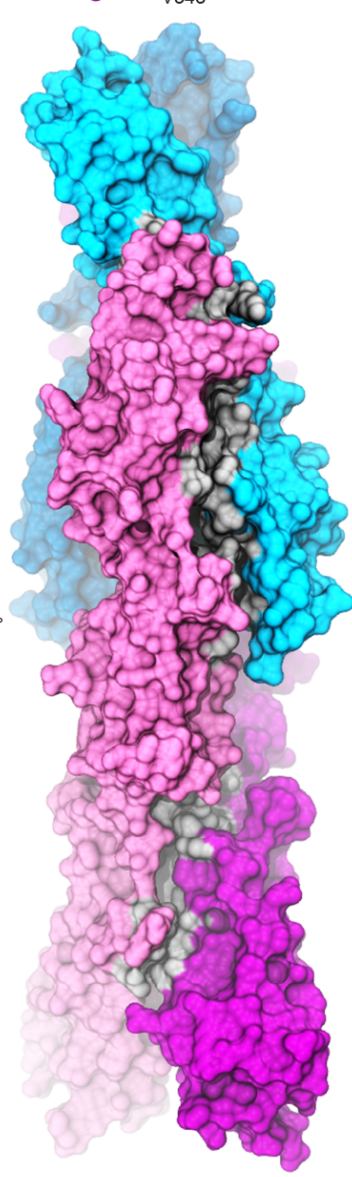
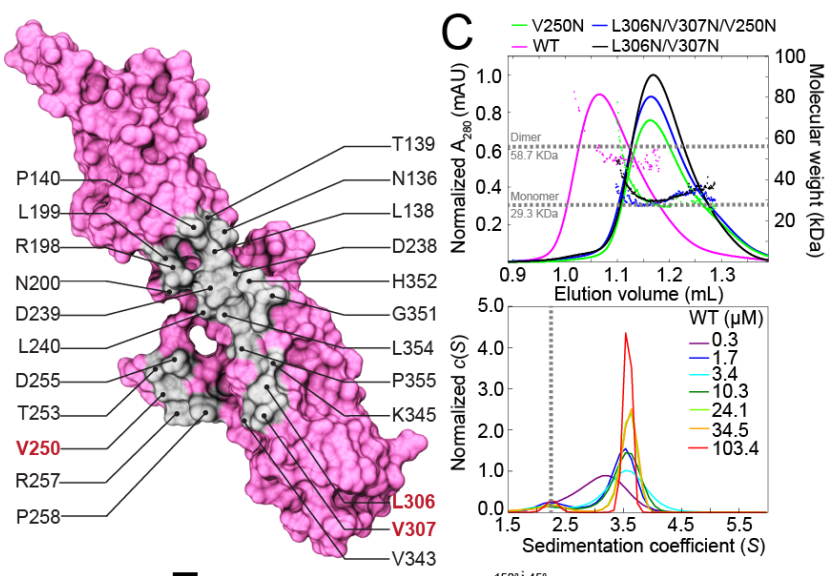

E

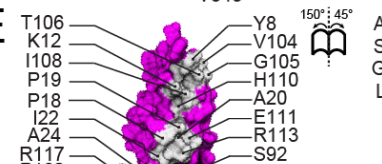

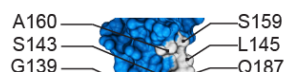
- 139 - 2187 R72 V99 $\quad \mathrm{G} 100$ S76- 77 - 18 Q98 Co स $-\mathrm{E} 15$

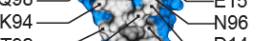
T92 — C 2014 Pan -F8 G89—// $\square$ - $\square$

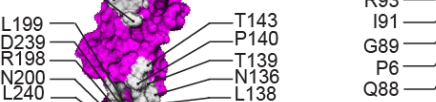
P355 ح $\quad \mathrm{D} 238$ V250 L S $\begin{aligned} & \mathrm{T} 253 \\ & \mathrm{~K} 3551 \\ & \mathrm{~K} 345\end{aligned}$

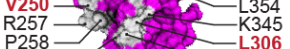

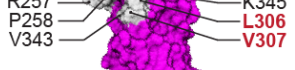
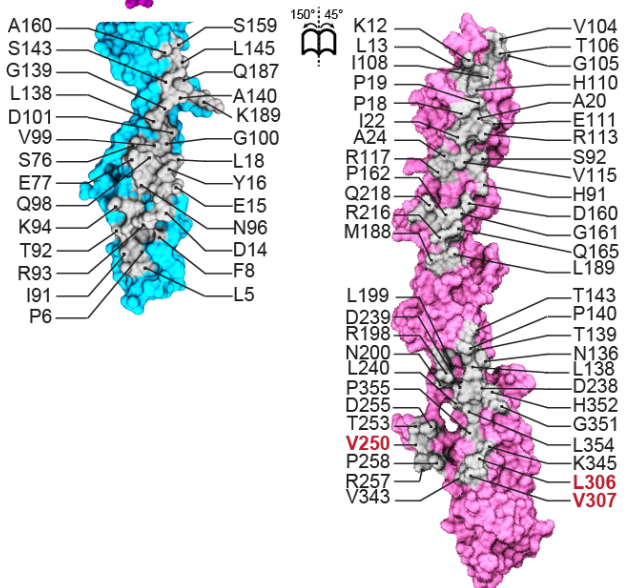

Figure 2. Dimerization of PCDH15 at the EC2-3 linker and the heterotetrameric tip-link bond. (A) Front view of the $h s$ PCDH15 EC2-3 dimer in surface representation (mauve and purple). Dimer interface is shown in silver. (B) Interaction surfaces exposed and colored as in A with interfacing residues labeled. Red labels indicate sites of mutations used to test interface. (C) Top panel shows SEC-MALS data for $h s$ PCDH15 EC2-3 WT fragment and a few representative mutants. Molecular mass is plotted in colored dots. Horizontal dashed lines indicate theoretical molar mass of $29.3 \mathrm{kDa}$ and $58.7 \mathrm{kDa}$ for monomer and dimer, respectively. Bottom panel shows $c(S)$ distribution from AUC-SV experiments using $h s$ PCDH15 EC2-3 at various concentrations. Peaks at $S \sim 3.5$ represent dimers. Vertical dashed line indicates expected position for monomer. Data for mutants is shown in Fig. S7. (D) Front and side views of the heterotetrameric $h s$ PCDH15 EC1-3 G16D/N369D/Q370N + $\mathrm{mm}$ CDH23 EC1-2 T15E structure. Molecular surfaces are shown as in A with CDH23 in cyan and blue. (E) Interaction surfaces exposed and colored as in $\mathrm{D}$ with interfacing residues labeled. 

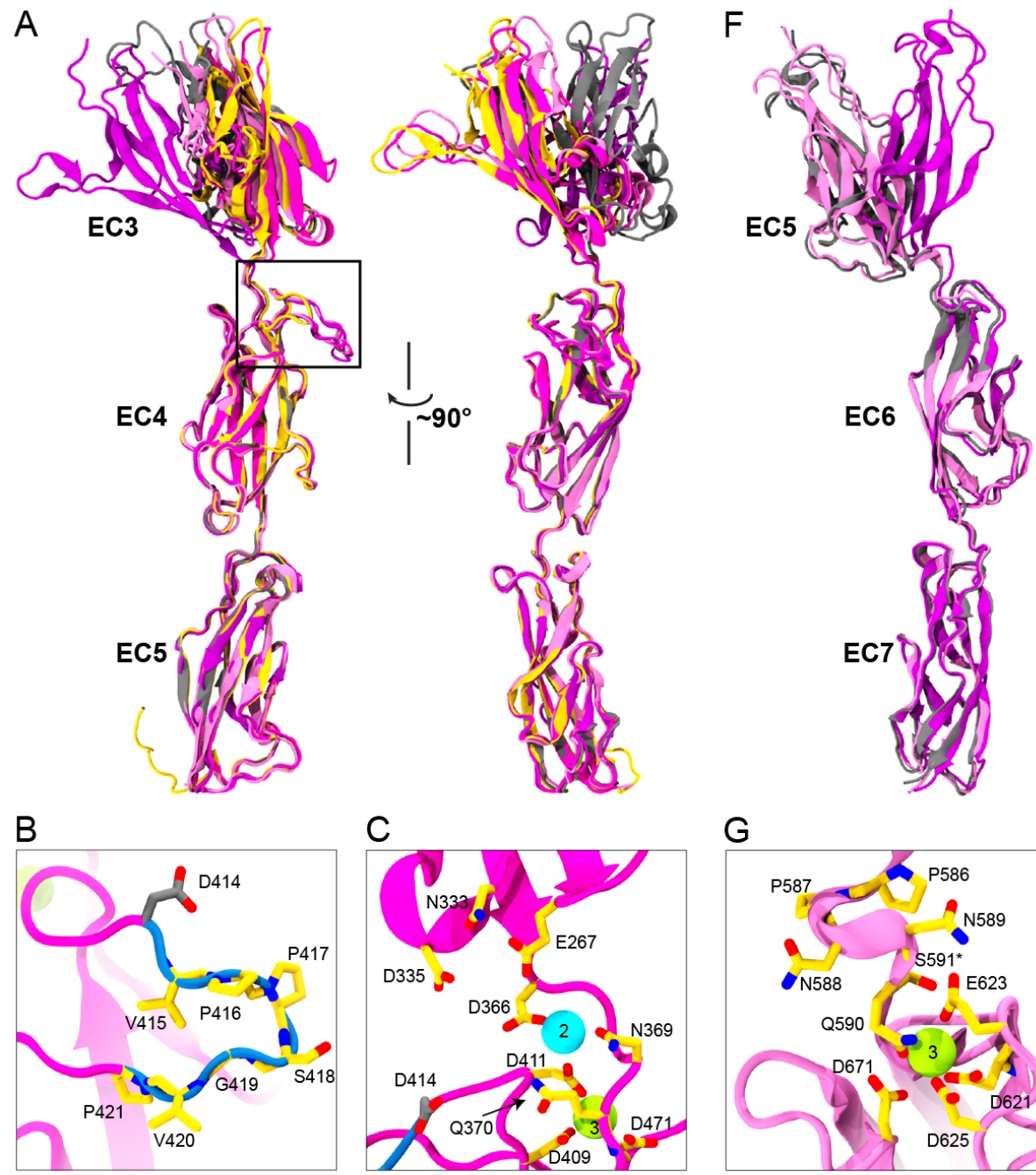

C

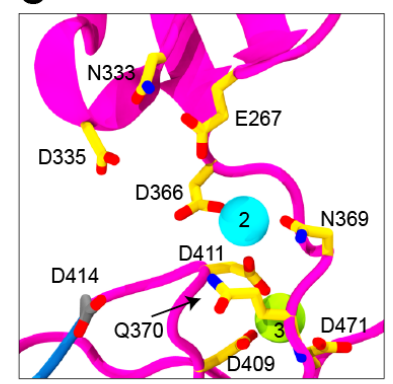

G
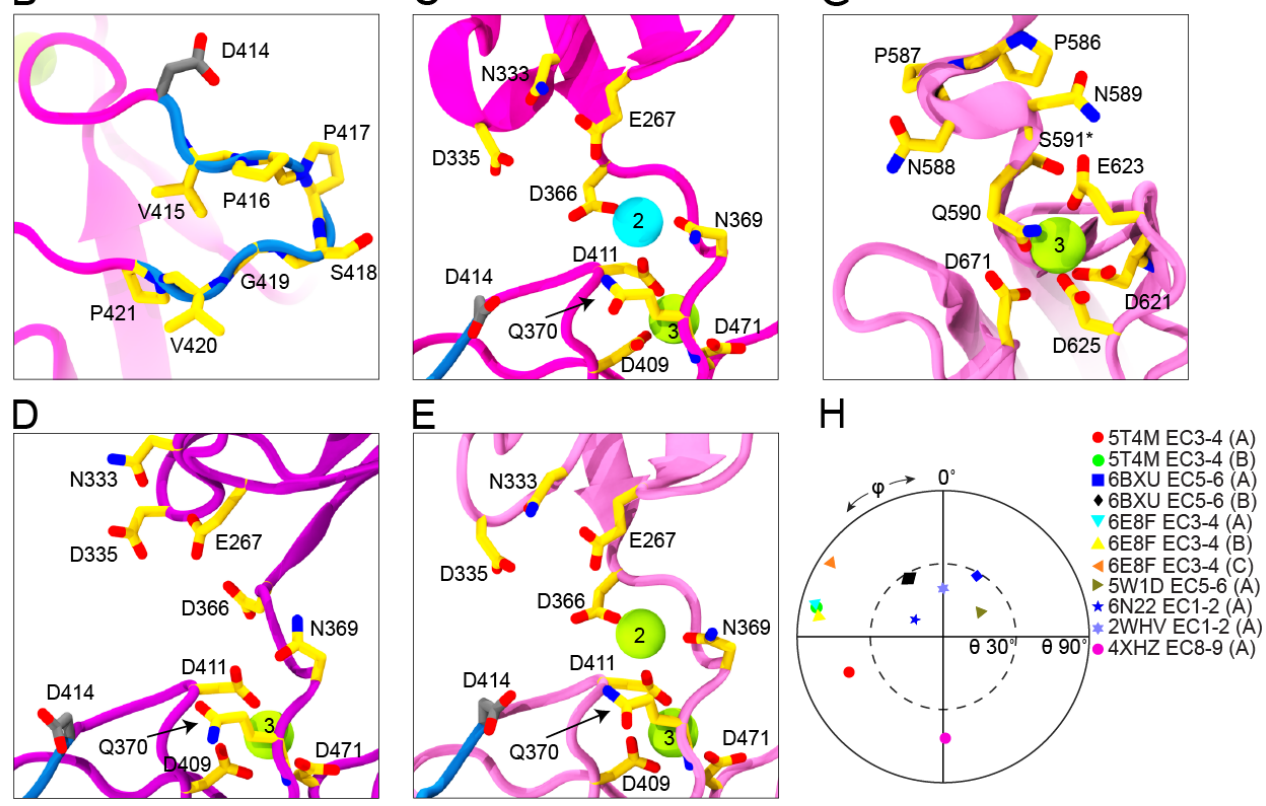

Figure 3. Flexibility of PCDH15 EC3-4 and EC5-6 linker regions. (A) Ribbon representation of $h s$ PCDH15 EC3-5 CD2-1 with protomers (mauve, purple, and magenta) superposed in two views illustrating flexibility at EC3-4. Also superposed are protomers of $h s$ PCDH15 EC3-5 CD1-1 (yellow and gray, PDB 5T4M ${ }^{44}$ ). Black box highlights location of CD2-1 insertion. (B) Detail of the insertion p.V(414+1)PPSGVP(414+7) encoded by exon 12a (blue backbone). Relevant residues are shown in stick representation and labeled. Some backbone atoms are omitted for clarity. Residue p.D414, thought to be under positive selection ${ }^{134}$ and involved in inherited deafness $^{135}$ is shown in gray. (C-E) Detail of $h s$ PCDH15 EC3-4 linker region for the three different protomers observed in the asymmetric unit of the $h s$ PCDH15 EC3-5 CD2-1 structure. Shown as in B. Sodium is in cyan. (F) Ribbon representation of $\mathrm{mm}$ PCDH15 EC5-7 with protomers from $\mathrm{mm}$ PCDH15 EC4-7 (gray) and from $\mathrm{mm}$ PCDH15 EC5-7 I582T (mauve and purple) superposed. (G) Detail of atypical $\mathrm{Ca}^{2+}$-binding linker region in $\mathrm{mm}$ PCDH15 EC5-6 with only one $\mathrm{Ca}^{2+}$ at site 3. (H) Orientation of tandem EC repeats for listed PCDH15 linker regions $(5 \mathrm{~T} 4 \mathrm{M}, 6 \mathrm{BXU}, 6 \mathrm{E} 8 \mathrm{~F}, 5 \mathrm{~W} 1 \mathrm{D}, 6 \mathrm{~N} 22$, 4XHZ) and CDH23 EC1-2 (2WHV). The N-terminal EC repeat for labeled structures was used as reference and aligned to the $z$ axis. The projection in the $x-y$ plane of the principal axis of the C-terminal EC repeat was plotted. $\mathrm{CDH} 23$ EC1-2 was used to define the azimuthal angle $\varphi$ as $0^{\circ}$. 


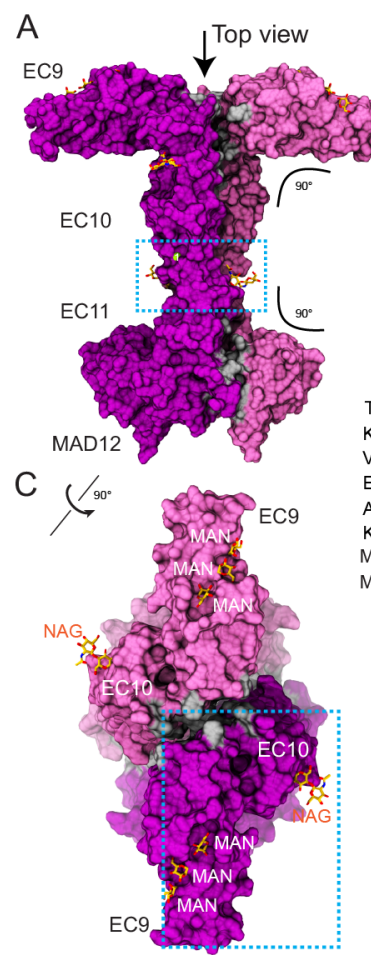

F
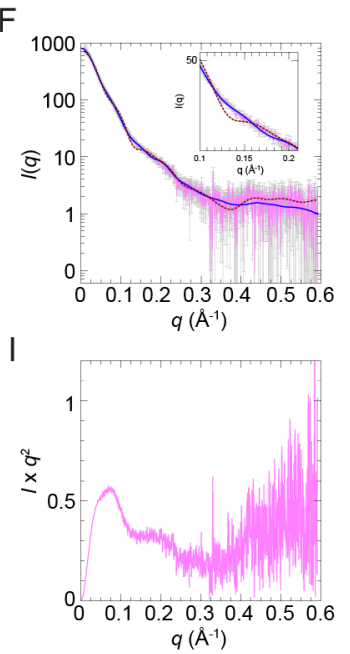
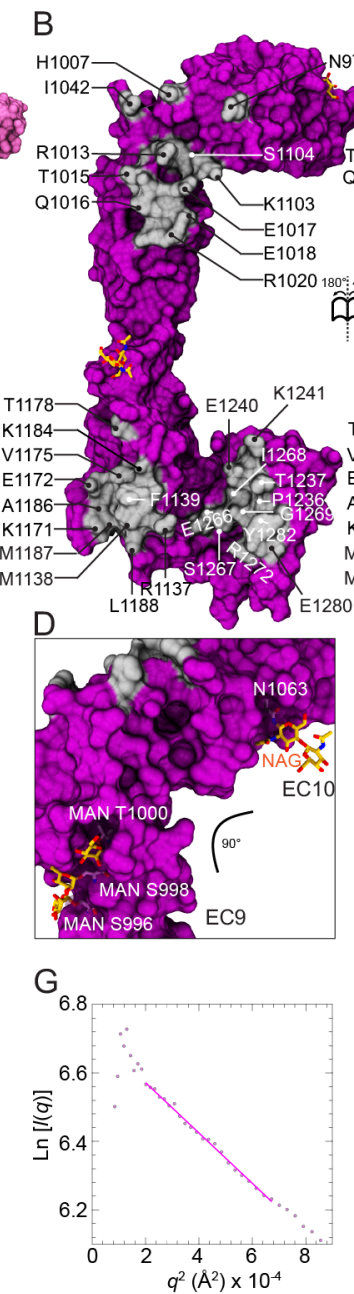

J

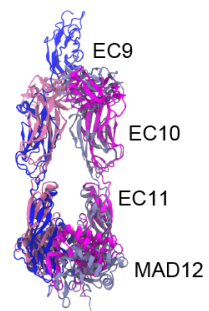

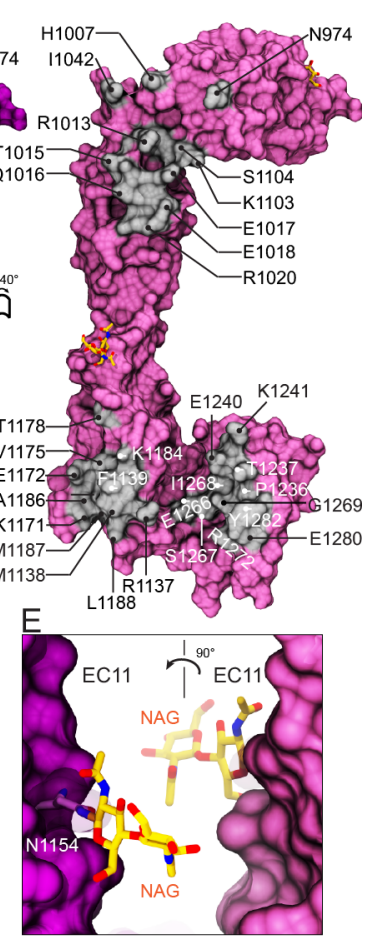

$\mathrm{H}$
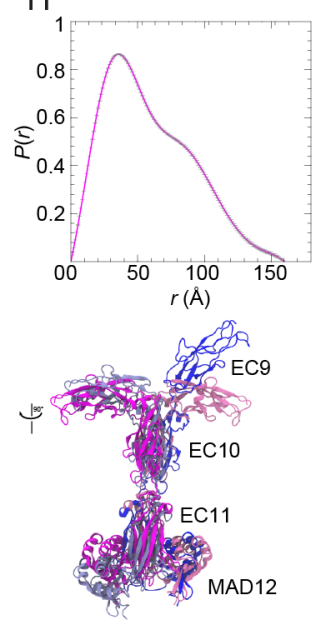

Figure 4. Dimerization of PCDH15 at the EC9-MAD12 region. (A) Molecular surface showing the glycosylated $\mathrm{mm}$ PCDH15 EC9-MAD12 parallel dimer in mauve and purple. Dimer interfaces are shown in gray, and glycosylation sugars are shown as yellow sticks. Blue dashed box denotes region highlighted in E. (B) Interaction surfaces exposed and colored as in A with interfacing residues labeled. (C) Top view of the $\mathrm{mm}$ PCDH15 EC9-MAD12 parallel dimer, with EC9 repeats pointing in opposite directions. Blue dashed box denotes region highlighted in D. (D-E) Details of glycosylation sites (dashed boxes in A and C). (F) X-ray scattering intensity as a function of the scattering vector $q$ (SAXS profile) for the bacterially produced $\mathrm{mm}$ PCDH15 EC9-MAD12. Predicted scattering intensities from the structure obtained with FoXS are shown in maroon-dashed lines $\left(\chi^{2}=6.52\right)$, while the theoretical scattering curve obtained from flexible refinement with SREFLEX is shown in blue $\left(\chi^{2}=1.38\right)$. (G) Guinier plot of the SAXS data in the low $q$ region. The magenta solid line shows the linear fit from which the gradient of the slope $\left(-R_{\mathrm{g}}{ }^{2} / 3\right)$ was used to estimate $R_{\mathrm{g}}$. (H) Realspace pair distribution function $P(r)$. (I) Kratky plot indicating the presence of folded and flexible protein. (J) Representative model of $\mathrm{mm}$ PCDH15 EC9-MAD12 from flexible refinement with SREFLEX (dark and light blue) superposed to the crystal structure of $\mathrm{mm}$ PCDH15 EC9-MAD12, magenta and mauve). 


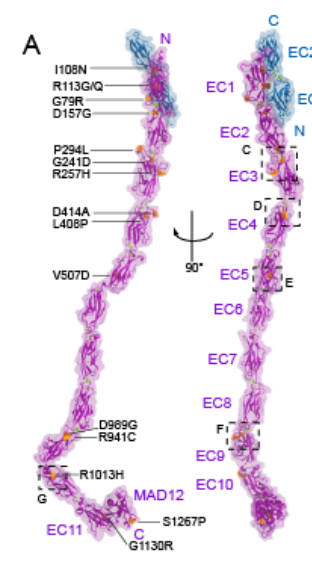

B

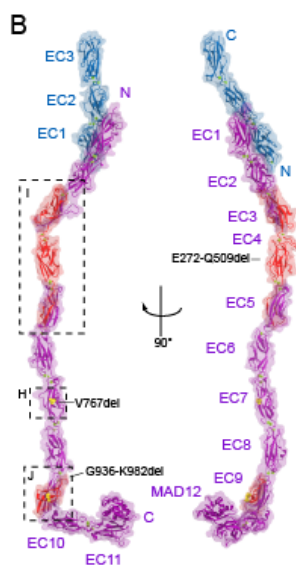

G
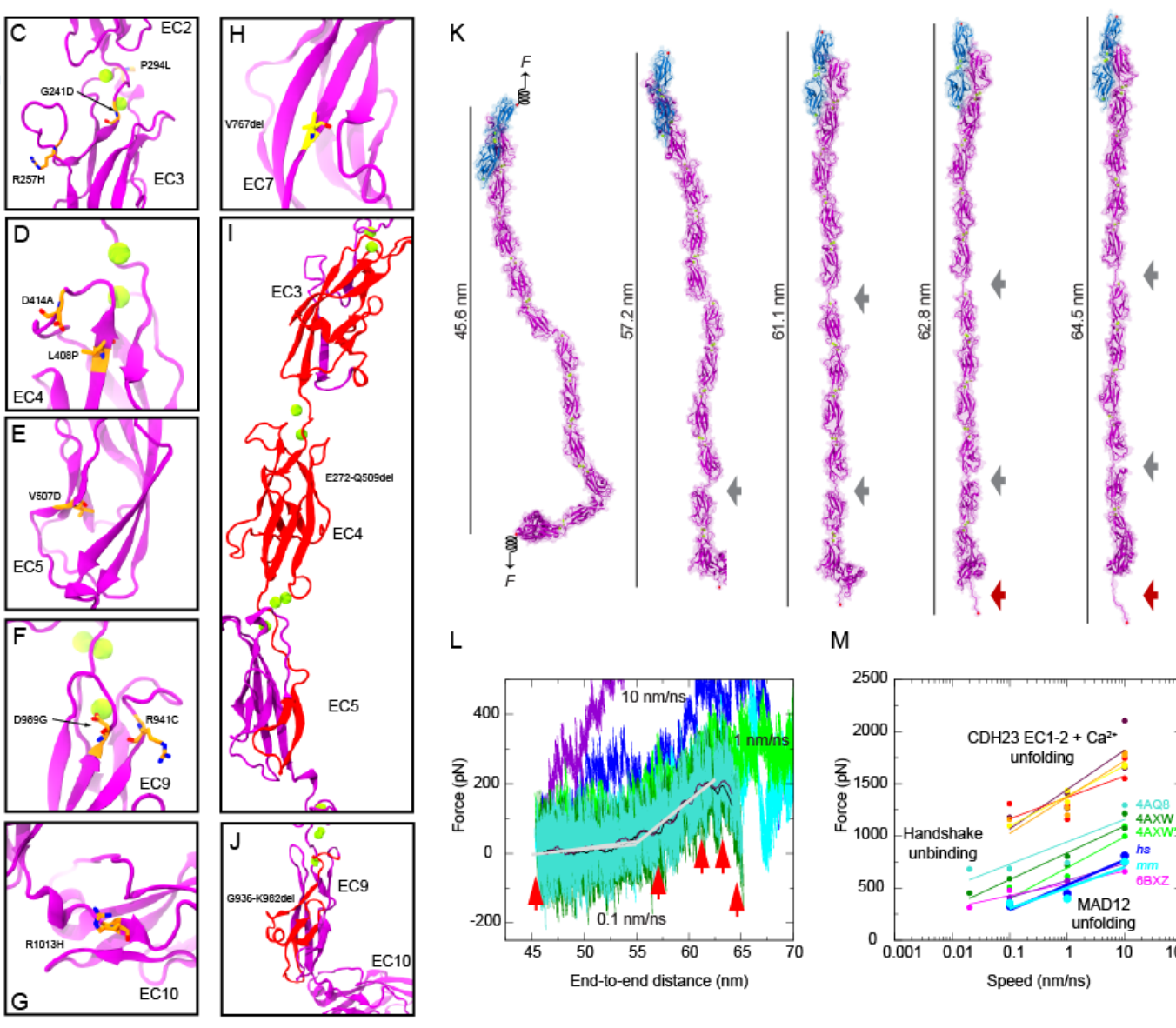

L

$\mathrm{M}$
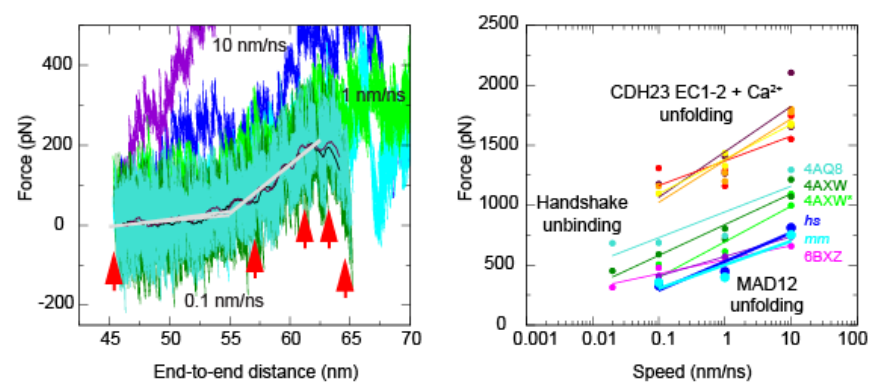

Figure 5. Disease-causing mutations and complete model and elasticity of the monomeric PCDH15 ectodomain. (A) Two views of the $h s$ PCDH15 EC1-MAD12 CD1-1 + CDH23 EC1-2 model with PCDH15 deafness-causing missense mutations listed. Proteins are shown in purple (PCDH15) and blue (CDH23) ribbon representations with transparent molecular surfaces. $\mathrm{Ca}^{2+}$ ions are shown as green spheres and mutations are highlighted in orange. Dashed boxes and labels correspond to select missense mutations shown in C-G panels. (B) The $m m$ PCDH15 EC1-MAD12 CD2-1 + CDH23 EC1-3 model with PCDH15 in-frame deletion mutations that cause deafness highlighted in red (multi-residue deletions) and yellow (single residue deletions). Dashed boxes and labels correspond to detailed views in H-J panels. (C-J) Detail of missense mutations and in-frame deletions that cause deafness. (K) Snapshots of the monomeric hs PCDH15 EC1-MAD12 CD1-1 + CDH23 EC1-2 system during stretching simulation S1d $(0.1 \mathrm{~nm} / \mathrm{ns}$, Table S8). Stretched C-terminal C $\alpha$ atoms are shown as red spheres. Springs in first panel indicate position and direction of applied forces. Gray arrows highlight stretching of PCDH15 EC linkers (EC9-10 followed by EC5-6). Dark red arrow indicates unfolding of PCDH15 MAD12's C-terminal end. (L) Force versus end-to-end distance for constant velocity stretching of $h s$ PCDH15 EC1-MAD12 + CDH23 EC1-2 at $10 \mathrm{~nm} / \mathrm{ns}$ (S1b, purple and blue), $1 \mathrm{~nm} / \mathrm{ns}$ (S1c, bright green and cyan), and $0.1 \mathrm{~nm} / \mathrm{ns}$ (S1d, dark green and turquoise; 10-ns running averages shown in black and maroon; gray lines are fits used to determine elasticity of the complex). Red arrowheads indicate time-points for S1d illustrated in K. (M) In silico force peak maxima versus stretching speed for CDH23 EC1-2 unfolding (red, maroon, yellow, and orange) ${ }^{42}$, for CDH23 EC1-2 and PCDH15 EC1-2 handshake unbinding (turquiose-4AQ8, dark green-4AXW, and light green-4AXW* [after a $1 \mu$ s-long equilibration] ${ }^{33,37}$, for PCDH15 EC10-MAD12 unfolding (magenta-6BXZ with unfolding at MAD12) ${ }^{40}$, for $h s$ PCDH15 EC1-MAD12 CD1-1 + CDH23 EC1-2 unfolding (S1b-d, blue with unfolding at MAD12), and for $m m$ PCDH15 EC1-MAD12 CD2-1 + CDH23 EC1-3 unfolding (S2b-d, cyan, with unfolding at MAD12). 

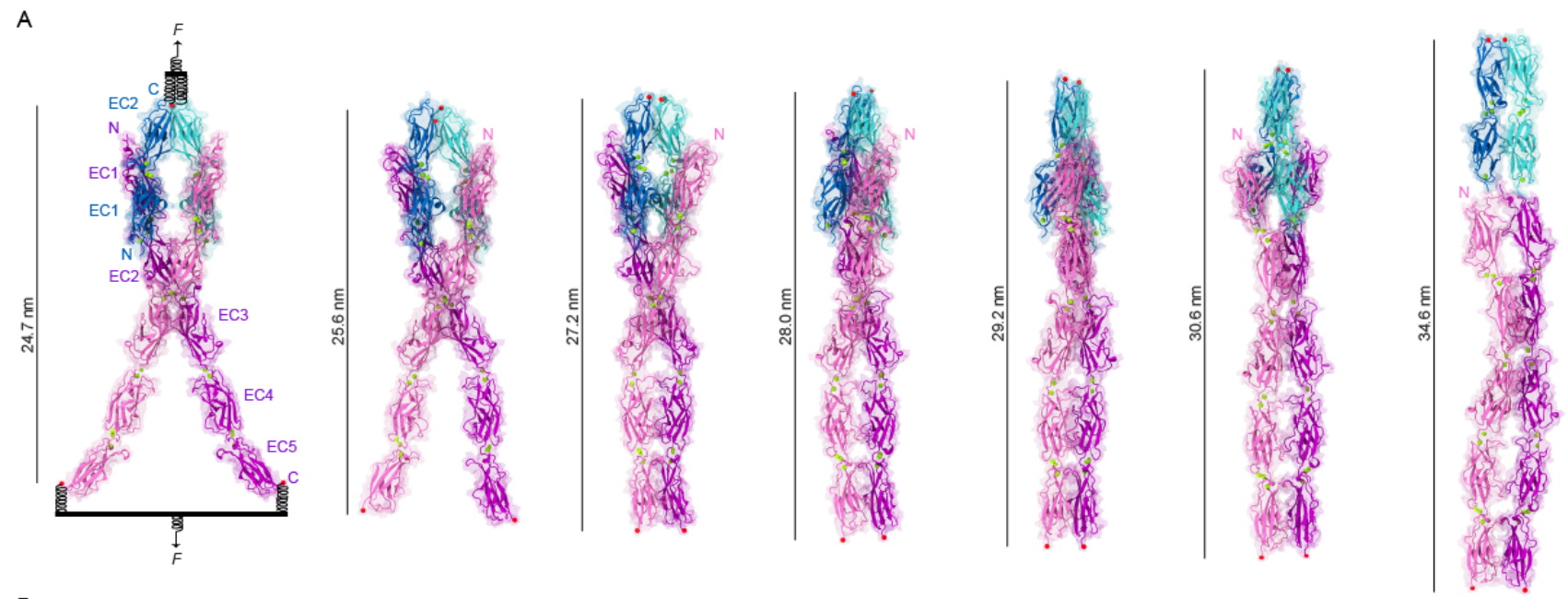

B
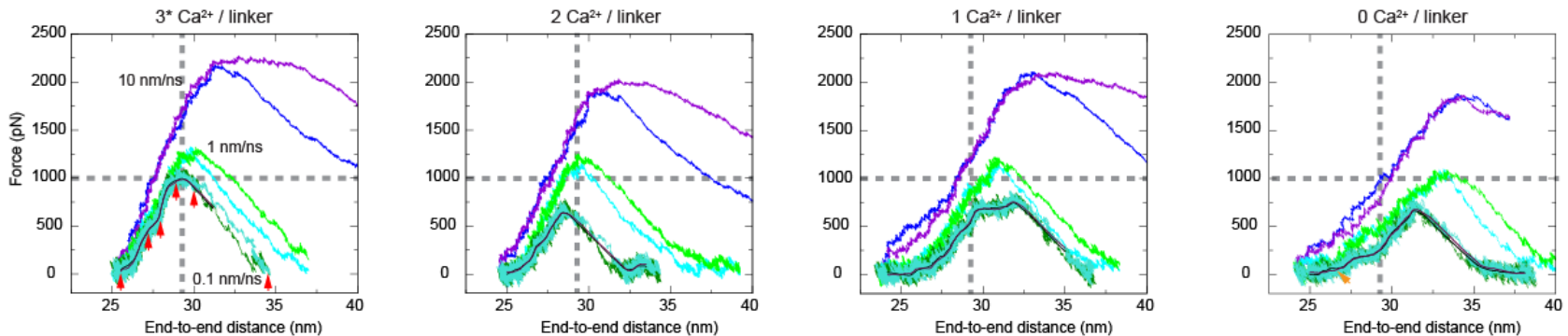

Figure 6. In silico strength of trans heterotetrameric bonds. (A) Snapshots of the heterotetrameric $h s$ $(\mathrm{PCDH} 15 \mathrm{EC} 1-\mathrm{EC} 5)_{2}+(\mathrm{CDH} 23 \mathrm{EC} 1-2)_{2}$ system during stretching simulation $\mathrm{S} 3 \mathrm{~d}(0.1 \mathrm{~nm} / \mathrm{ns}$, Table S8). Stretched C-terminal $\mathrm{C} \alpha$ atoms are shown as red spheres. Stretching was carried out by attaching two slabs to springs that were in turn attached to the terminal ends of each protein protomer. Slabs were moved in opposite directions at constant speed through individual springs. (B) Force applied to each of the slabs versus protein separation (see Methods) for constant velocity stretching simulations of $h s$ (PCDH15 EC1-EC5) $2+(\mathrm{CDH} 23$ EC1-2) $)_{2}$ systems with all $\mathrm{Ca}^{2+}$-binding sites occupied (S3b-d; $3 \mathrm{Ca}^{2+}$ ions per linker except for PCDH15 EC2-3 and EC3-4 linkers), with $2 \mathrm{Ca}^{2+}$ ions per linker (S4b-d; $\mathrm{Ca}^{2+}$-binding sites 2 and 3), with $1 \mathrm{Ca}^{2+}$ ion per linker (S5b-d; $\mathrm{Ca}^{2+}$-binding site 3), and without bound $\mathrm{Ca}^{2+}$ ions (S6b-d). Traces are for constant velocity simulations at $10 \mathrm{~nm} / \mathrm{ns}$ (purple and blue), $1 \mathrm{~nm} / \mathrm{ns}$ (bright green and cyan), and $0.1 \mathrm{~nm} / \mathrm{ns}$ (dark green and turquoise with 10-ns running averages in black and maroon). Gray dashed lines indicate magnitude and position of force peak at the slowest stretching speed tested with all $\mathrm{Ca}^{2+}$-binding sites occupied. Red arrowheads in leftmost panel indicate time-points for simulation S3d illustrated in A. Orange arrowhead in rightmost panel indicates soft elastic response region. 
A

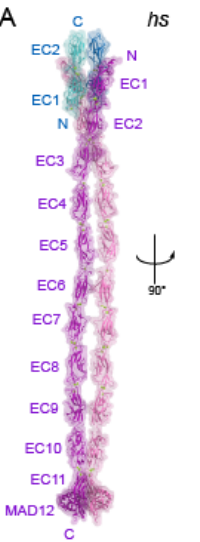

E

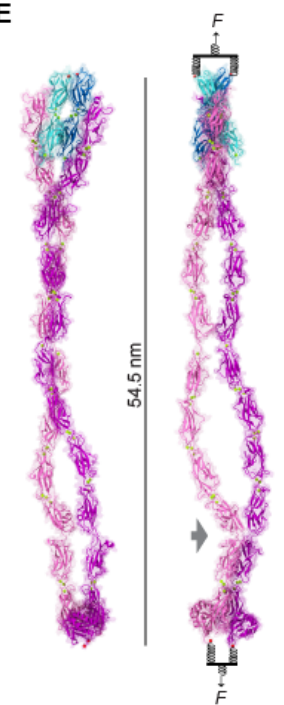

B
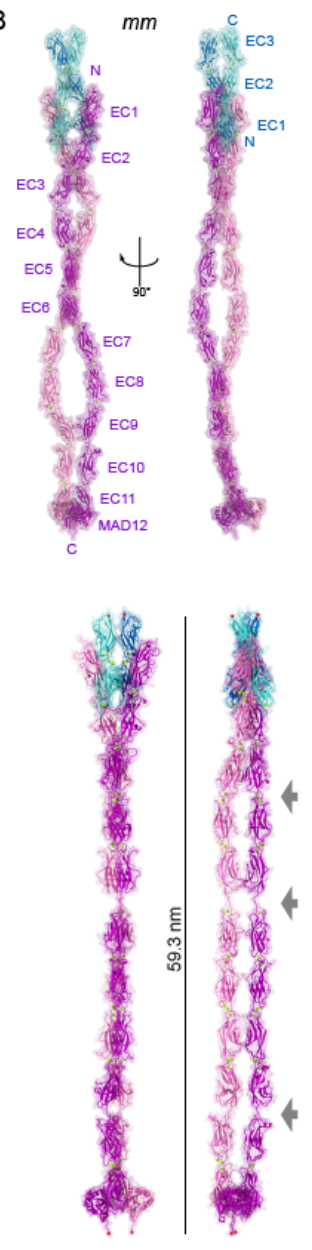

C
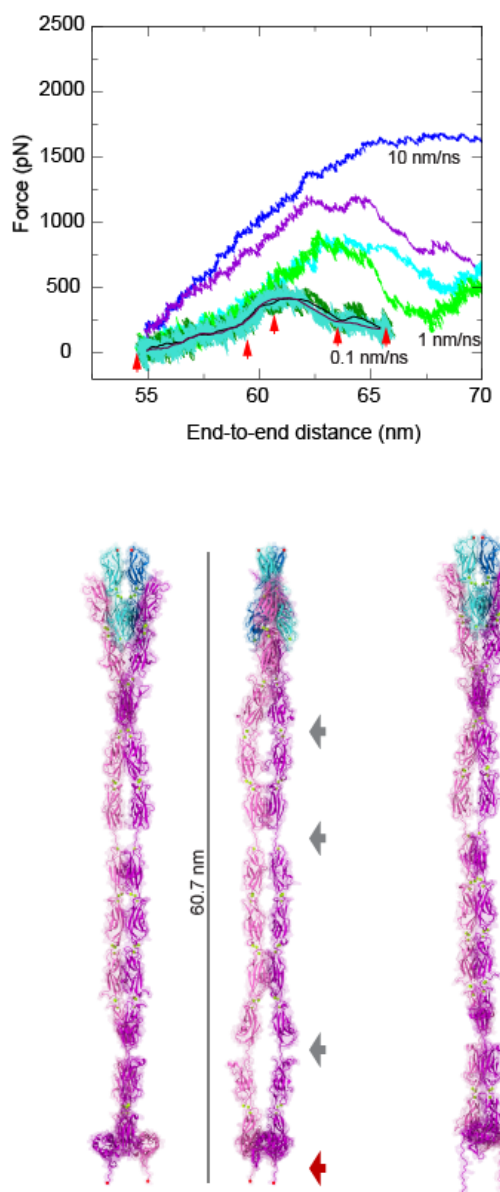

D

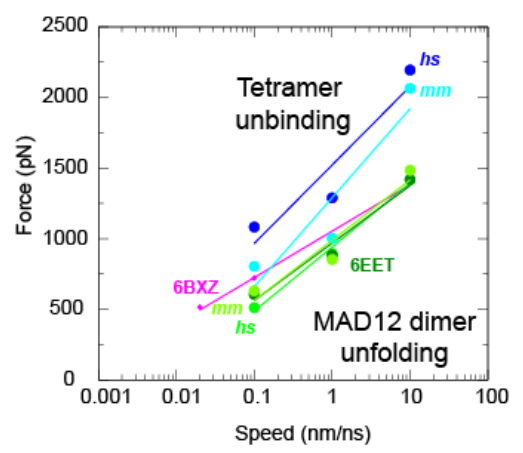

Figure 7. Models and mechanics of the PCDH15 ectodomain as part of an heterotetrameric complex with CDH23. (A-B) Two views of the (A) $h s$ (PCDH15 EC1-MAD12) $)_{2}+(\mathrm{CDH} 23 \mathrm{EC} 1-2)_{2}$ and (B) $m m$ (PCDH15 EC1-MAD12 $)_{2}+(\mathrm{CDH} 23 \text { EC1-3 })_{2}$ heterotetrameric systems before simulation. (C) Force applied to slabs versus protein separation (see Methods and Table S8) for constant velocity stretching simulations of the $h s$ $(\mathrm{PCDH} 15 \mathrm{EC} 1-\mathrm{MAD} 12)_{2}+(\mathrm{CDH} 23 \mathrm{EC} 1-2)_{2}$ system at $10 \mathrm{~nm} / \mathrm{ns}(\mathrm{S} 8 \mathrm{~b}$, purple and blue), $1 \mathrm{~nm} / \mathrm{ns}$ (S8c, bright green and cyan), and $0.1 \mathrm{~nm} / \mathrm{ns}$ (S8d, dark green and turquoise with 10-ns running averages in black and maroon). Red arrowheads indicate time-points for simulation S8d illustrated in E. (D) In silico force peak maxima versus stretching speed for $h s$ (PCDH15 EC1-EC5) $)_{2}+(\mathrm{CDH} 23 \text { EC1-2) })_{2}$ (S3b-d, blue), for PCDH15 EC10-MAD12 unfolding (magenta-6BXZ with unfolding at MAD12) ${ }^{40}$, for $\mathrm{mm}$ PCDH15 EC9-MAD12 dimer unfolding (S11b-d, dark green with unfolding at MAD12), and for $h s$ (PCDH15 EC1-MAD12) $+(\mathrm{CDH} 23$ EC1$2)_{2}$ unfolding (S8b-d, bright green with unfolding at MAD12). (E) Snapshots of the heterotetrameric $h s$ $(\mathrm{PCDH} 15 \mathrm{EC} 1-\mathrm{MAD} 12)_{2}+(\mathrm{CDH} 23 \mathrm{EC} 1-2)_{2}$ system during stretching simulation S8d $(0.1 \mathrm{~nm} / \mathrm{ns})$. Stretched Cterminal $\mathrm{C} \alpha$ atoms are shown as red spheres. Stretching was carried out by attaching two slabs to springs that were in turn attached to the terminal ends of each protein protomer. Slabs were moved in opposite directions at constant speed through individual springs. Gray arrows highlight stretching of PCDH15 EC linkers (EC9-10 followed by EC5-6) and re-bending at EC9-10. Dark red arrow indicates unfolding of PCDH15 MAD12's Cterminal end. 


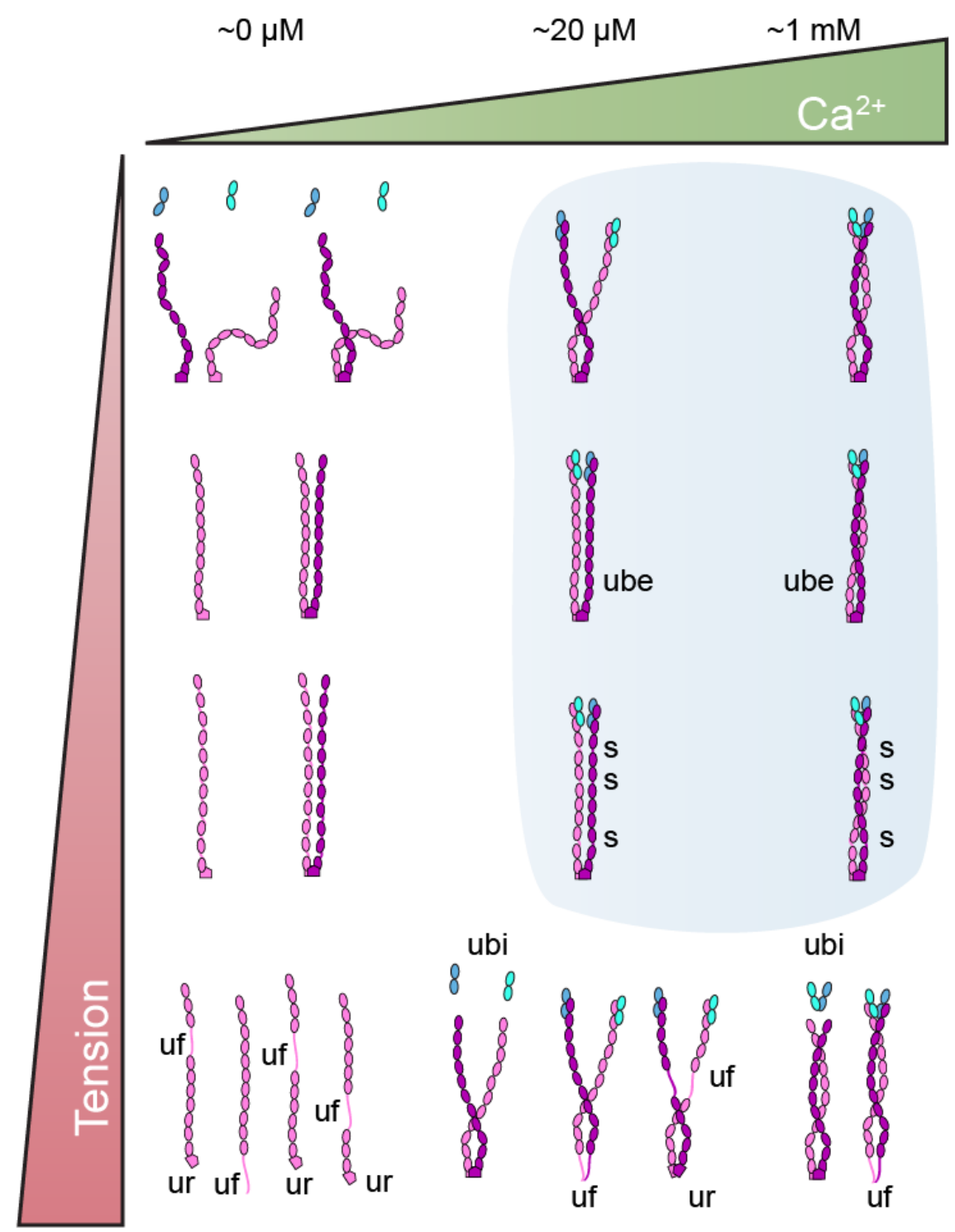

Figure 8. Tuning the elastic response of PCDH15. Structures and simulations indicate that the PCDH15 ectodomain can have distinct elastic responses that are tuned by tension and $\mathrm{Ca}^{2+}$. Diagram shows possible hypothetical states for PCDH15's ectodomain. At very low $\mathrm{Ca}^{2+}$ concentrations (top left) PCDH15 will not interact with $\mathrm{CDH} 23$ and its linkers will be flexible and easily stretchable under tension. At high tension and very low $\mathrm{Ca}^{2+}$ concentrations (lower left), the $\mathrm{Ca}^{2+}$-free linkers will be weak and unfolding (uf) at any of the EC repeats will ensue. Under normal physiological conditions (blue shade), PCDH15 will form a cis dimer that interacts with $\mathrm{CDH} 23$ and that can exhibit soft elasticity mediated by bending and unbending transitions of $\mathrm{Ca}^{2+}-$ free linker regions EC5-6 and EC9-10. As tension increases, unbending (ube) results in stiffening of PCDH15. Stretching of linkers (s) and unrolling of MAD12 (ur) at higher tension may soften the elastic response of PCDH15 for the subsequent stimulus. Under more extreme tension (bottom right) unbinding of PCDH15 from CDH23 (ubi) or unfolding of PCDH15 (uf) may occur. Glycosylation and specifics of PCDH15 splice isoforms may also influence the tip-link state and elastic response. The effect of resting tension is further dissected in Fig. S22. 
bioRxiv preprint doi: httos://doi ora/101101/695502: this version posted Julv 8 2019. The copvriaht holder for this preprint (which was not certified by peer review) is the author/funder, who has granted bioRxiv a license to display the preprint in perpetuity. It is made available under aCC-BY-NC-ND 4.0 International license.

\section{TABLES}

Table 1. Statistics for structures

\begin{tabular}{|c|c|c|c|c|}
\hline Data collection & $\begin{array}{l}\text { Mm PCDH15 EC1-2 } \\
\text { BAP }\end{array}$ & $\begin{array}{l}\text { Hs PCDH15 EC1-3 } \\
\text { G16D/N369D/ } \\
\text { Q370N }\end{array}$ & $\begin{array}{l}\text { Hs PCDH15 EC1-3 G16D } \\
\text { /N369D/Q370N + Mm } \\
\text { CDH23 EC1-2 T15E }^{\#}\end{array}$ & Hs PCDH15 EC2-3 \\
\hline Space group & $\mathrm{P}_{4}$ & $\mathrm{C} 222_{1}$ & $\mathrm{P} 2_{1}$ & $\mathrm{P} 2_{1}$ \\
\hline \multicolumn{5}{|l|}{ Unit cell parameters } \\
\hline$a, b, c(\AA)$ & $99.62,99.62,58.56$ & $\begin{array}{l}87.89,116.52, \\
99.89\end{array}$ & $76.79,65.40,190.02$ & $\begin{array}{l}65.76,147.63, \\
77.03\end{array}$ \\
\hline Beam source & APS-24-ID-C & APS-24-ID-C & APS-24-ID-E & APS-24-ID-C \\
\hline Date of data collection & 21-MAR-2015 & 07-NOV-2017 & 18-OCT-2018 & 21-MAR-2015 \\
\hline Wavelength $(\AA ̊)$ & 0.9792 & 0.9792 & 0.9792 & 0.9792 \\
\hline Resolution limit ( $\AA$ ) & 2.40 & 3.15 & 2.90 & 2.64 \\
\hline Unique reflections & 13,043 & 9,312 & 41,847 & 42,598 \\
\hline$R_{\text {merge }}$ & $0.13(0.80)$ & $0.36(1.38)$ & $0.12(0.47)$ & $0.10(0.58)$ \\
\hline$R_{\text {meas }}$ & $0.14(0.86)$ & $0.38(1.53)$ & $0.13(0.53)$ & $0.12(0.73)$ \\
\hline$R_{\text {pim }}$ & $0.05(0.31)$ & $0.12(0.62)$ & $0.06(0.24)$ & $0.07(0.44)$ \\
\hline$C C_{1 / 2}$ & $0.97(0.90)$ & $0.95(0.34)$ & $0.98(0.94)$ & $0.90(0.65)$ \\
\hline$C C^{*}$ & $0.99(0.97)$ & $0.99(0.71)$ & $0.99(0.99)$ & $0.97(0.89)$ \\
\hline \multicolumn{5}{|l|}{ Refinement } \\
\hline \multirow[t]{2}{*}{ Resolution range $(\AA)$} & $49.86-2.40$ & $49.99-3.15$ & $49.52-2.90$ & $50.00-2.64$ \\
\hline & $(2.47-2.40)$ & $(3.20-3.15)$ & $(2.97-2.90)$ & $(2.70-2.64)$ \\
\hline$R_{\text {work }}(\%)$ & $17.0(23.0)$ & $21.5(27.9)$ & $22.5(29.9)$ & $21.5(33.6)$ \\
\hline$R_{\text {free }}(\%)$ & $23.3(29.8)$ & $28.8(36.3)$ & $25.3(32.4)$ & $24.6(31.5)$ \\
\hline Ligand/ion & 38.38 & 57.79 & 81.57 & 39.89 \\
\hline Water & 42.48 & 46.60 & 50.95 & 37.60 \\
\hline \multicolumn{5}{|c|}{ Ramachandran Plot Region (PROCHECK) } \\
\hline Most favored (\%) & 89.1 & 82.4 & 88.9 & 90.4 \\
\hline Additionally allowed (\%) & 10.4 & 17.2 & 10.9 & 9.4 \\
\hline Generously allowed (\%) & 0.5 & 0.4 & 0.2 & 0.1 \\
\hline Disallowed (\%) & 0.0 & 0.0 & 0.0 & 0.0 \\
\hline PDB ID & 6 N22 & 6MFO & 6N2E & 5ULY \\
\hline
\end{tabular}

"Amplitude-based twin refinement. 
bioRxiv preprint doi: https://doi org/10 1101/695502; this version posted July 8 2019. The copyright holder for this preprint (which was not certified by peer review) is the author/funder, who has granted bioRxiv a license to display the preprint in perpetuity. It is made available under aCC-BY-NC-ND 4.0 International license.

Table 1. Statistics for structures (continued)

\begin{tabular}{|c|c|c|c|c|}
\hline Data collection & $\begin{array}{l}\text { Hs PCDH15 EC2-3 } \\
\text { V250N }\end{array}$ & $\begin{array}{l}\text { Hs PCDH15 EC3-5 } \\
\text { CD2-1 }\end{array}$ & Mm PCDH15 EC4-7 & $\begin{array}{l}\text { Mm PCDH15 EC5-7 } \\
\text { I582T }\end{array}$ \\
\hline Space group & $\mathrm{P} 2_{1}$ & $\mathrm{P} 222_{1}$ & $\mathrm{P} 4_{3} 2_{1} 2$ & $\mathrm{P}_{2} 22$ \\
\hline \multicolumn{5}{|l|}{ Unit cell parameters } \\
\hline$a, b, c(\AA)$ & $\begin{array}{l}77.18,31.95 \\
115.96\end{array}$ & $\begin{array}{l}95.74,95.91 \\
258.60\end{array}$ & $\begin{array}{l}78.86,78.86 \\
289.47\end{array}$ & $\begin{array}{l}\text { 180.91, 180.91, } \\
127.16\end{array}$ \\
\hline$\alpha, \beta, \gamma\left(^{\circ}\right)$ & $90.0,106.4,90.0$ & $90.0,90.0,90.0$ & $90.0,90.0,90.0$ & $90.0,90.0,90.0$ \\
\hline Molecules per asymmetric unit & 2 & 3 & 1 & 2 \\
\hline Beam source & APS-24-ID-E & APS-24-ID-C & APS-24-ID-C & APS-24-ID-E \\
\hline Date of data collection & 13-FEB-2018 & 29-MAR-2018 & 10-JUL-2015 & 19-DEC-2016 \\
\hline Wavelength $(\AA ̊)$ & 0.9792 & 0.9792 & 0.9792 & 0.9792 \\
\hline Resolution limit $(\AA ̊)$ & 2.60 & 2.99 & 3.35 & 3.79 \\
\hline Unique reflections & 17,491 & 48,387 & 14,064 & 21,629 \\
\hline Completeness (\%) & $95.0(89.2)$ & $99.9(99.1)$ & $98.5(86.4)$ & $97.6(87.8)$ \\
\hline Redundancy & $4.2(2.8)$ & $10.2(7.9)$ & $11.2(7.2)$ & $6.4(4.7)$ \\
\hline$I / \sigma(I)$ & $7.3(2.9)$ & $9.3(2.4)$ & $16.9(2.5)$ & $7.43(0.55)$ \\
\hline$R_{\text {merge }}$ & $0.19(0.33)$ & $0.21(0.81)$ & $0.13(0.60)$ & $0.23(2.43)$ \\
\hline$R_{\text {meas }}$ & $0.22(0.39)$ & $0.22(0.87)$ & $0.14(0.64)$ & 0.25 (2.69) \\
\hline$R_{\text {pim }}$ & $0.10(0.20)$ & $0.07(0.30)$ & $0.04(0.21)$ & $0.10(1.12)$ \\
\hline$C C_{1 / 2}$ & $0.867(0.897)$ & $(0.782)$ & $0.99(0.91)$ & $0.77(0.27)$ \\
\hline$C C^{*}$ & $0.964(0.972)$ & (0.937) & $0.99(0.98)$ & $0.91(0.65)$ \\
\hline \multicolumn{5}{|l|}{ Refinement } \\
\hline \multirow[t]{2}{*}{ Resolution range $(\AA)$} & $39.47-2.60$ & $47.95-2.99$ & $76.09-3.35$ & $180.90-3.79$ \\
\hline & $(2.65-2.58)$ & $(3.07-2.99)$ & $(3.44-3.35)$ & $(3.88-3.79)$ \\
\hline$R_{\text {work }}(\%)$ & $23.2(31.7)$ & $17.4(23.4)$ & $22.7(39.2)$ & $24.0(37.9)$ \\
\hline$R_{\text {free }}(\%)$ & $27.0(35.1)$ & $21.4(27.9)$ & $27.9(44.0)$ & $28.3(42.8)$ \\
\hline Residues (atoms) & $457(3,626)$ & $1021(7,966)$ & $405(3,118)$ & $623(4,756)$ \\
\hline Water molecules & 40 & 8 & 1 & - \\
\hline \multicolumn{5}{|l|}{ Rms deviations } \\
\hline Bond lengths $(\AA ̊)$ & 0.007 & 0.007 & 0.011 & 0.009 \\
\hline Bond angles $\left({ }^{\circ}\right)$ & 1.198 & 1.249 & 1.620 & 1.486 \\
\hline \multicolumn{5}{|l|}{$B$-factor average } \\
\hline Protein & 43.08 & 89.12 & 124.15 & 154.78 \\
\hline Ligand/ion & 41.04 & 85.04 & 107.63 & 111.88 \\
\hline Water & 28.69 & 58.58 & 91.23 & - \\
\hline \multicolumn{5}{|c|}{ Ramachandran Plot Region (PROCHECK) } \\
\hline Most favored (\%) & 90.6 & 88.1 & 84.6 & 86.2 \\
\hline Additionally allowed (\%) & 8.9 & 11.7 & 15.1 & 13.8 \\
\hline Generously allowed (\%) & 0.5 & 0.2 & 0.3 & 0.0 \\
\hline Disallowed (\%) & 0.0 & 0.0 & 0.0 & 0.0 \\
\hline PDB ID & 6EB5 & 6E8F & 5W1D & 6BXU \\
\hline
\end{tabular}

\# Amplitude-based twin refinement. 
bioRxiv preprint doi: https://doi.org/101101/695502; this version posted July 8 2019. The copyright holder for this preprint (which was not certified by peer review) is the author/funder, who has granted bioRxiv a license to display the preprint in perpetuity. It is made available under aCC-BY-NC-ND 4.0 International license.

Table 1. Statistics for structures (continued)

\begin{tabular}{|c|c|c|c|}
\hline Data collection & Mm PCDH15 EC6-7 & Mm PCDH15 EC7-8 V875A & Mm PCDH15 EC9-MAD12 \\
\hline Space group & $\mathrm{P}_{2} 22$ & $\mathrm{C} 2$ & $\mathrm{C} 222_{1}$ \\
\hline \multicolumn{4}{|l|}{ Unit cell parameters } \\
\hline$a, b, c(\AA)$ & $110.98,110.98,206.02$ & $135.80,22.99,70.74$ & $129.51,170.06,91.53$ \\
\hline$\alpha, \beta, \gamma\left({ }^{\circ}\right)$ & $90.0,90.0,120.0$ & $90.0,97.3,90.0$ & $90.0,90.0,90.0$ \\
\hline Molecules per asymmetric unit & 1 & 1 & 1 \\
\hline Beam source & APS-24-IDE-E & APS-24-ID-C & APS-24-ID-E \\
\hline Date of data collection & 15-JUN-2017 & 22-MAR-2015 & 02-JUL-2018 \\
\hline Wavelength $(\AA)$ & 0.9792 & 0.9792 & 0.9792 \\
\hline Resolution limit $(\AA)$ & 2.94 & 2.00 & 3.23 \\
\hline Unique reflections & 16,643 & 15,280 & 16,630 \\
\hline Completeness (\%) & $98.1(96.9)$ & $96.5(82.1)$ & $99.5(94.8)$ \\
\hline Redundancy & $7.7(5.2)$ & $3.1(2.4)$ & $9.8(4.7)$ \\
\hline$I / \sigma(I)$ & $10.92(2.00)$ & $23.1(5.4)$ & $14.5(2.2)$ \\
\hline$R_{\text {merge }}$ & $0.13(0.81)$ & $0.05(0.14)$ & $0.17(0.81)$ \\
\hline$R_{\text {meas }}$ & $0.14(0.89)$ & $0.05(0.18)$ & $0.18(0.89)$ \\
\hline$R_{\text {pim }}$ & $0.05(0.37)$ & $0.03(0.10)$ & $0.05(0.36)$ \\
\hline$C C_{1 / 2}$ & $0.98(0.89)$ & $0.99(0.97)$ & $1.01(0.78)$ \\
\hline$C C^{*}$ & $0.99(0.97)$ & $0.99(0.99)$ & $1.00(0.94)$ \\
\hline \multicolumn{4}{|l|}{ Refinement } \\
\hline \multirow[t]{2}{*}{ Resolution range $(\AA)$} & $96.11-2.94$ & $70.17-2.00$ & $45.81-3.23$ \\
\hline & $(3.02-2.94)$ & $(2.05-2.00)$ & $(3.31-3.23)$ \\
\hline$R_{\text {work }}(\%)$ & $21.7(40.3)$ & $19.0(21.1)$ & $17.6(27.8)$ \\
\hline$R_{\text {free }}(\%)$ & $24.2(42.4)$ & $22.9(27.5)$ & $23.4(47.0)$ \\
\hline Residues (atoms) & $206(1,594)$ & $202(1,586)$ & $443(3,476)$ \\
\hline Water molecules & 5 & 121 & 0 \\
\hline \multicolumn{4}{|l|}{ Rms deviations } \\
\hline Bond lengths $(\AA ̊)$ & 0.008 & 0.008 & 0.008 \\
\hline Bond angles $\left({ }^{\circ}\right)$ & 1.321 & 1.315 & 1.397 \\
\hline \multicolumn{4}{|l|}{$B$-factor average } \\
\hline Protein & 107.22 & 40.86 & 97.32 \\
\hline Ligand/ion & 82.53 & 44.45 & 127.92 \\
\hline Water & 74.82 & 41.45 & - \\
\hline \multicolumn{4}{|c|}{ Ramachandran Plot Region (PROCHECK) } \\
\hline Most favored (\%) & 87.2 & 93.8 & 86.8 \\
\hline Additionally allowed (\%) & 12.2 & 5.7 & 13.0 \\
\hline Generously allowed (\%) & 0.6 & 0.6 & 0.3 \\
\hline Disallowed (\%) & 0.0 & 0.0 & 0.0 \\
\hline PDB ID & 6BWN & 5TPK & 6EET \\
\hline
\end{tabular}


bioRxiv preprint doi: https://doi.org/10.1101/695502; this version posted July 8 2019. The copyright holder for this preprint (which was not certified by peer review) is the author/funder, who has granted bioRxiv a license to display the preprint in perpetuity. It is made available under aCC-BY-NC-ND 4.0 International license.

Table 2. Oligomerization state of PCDH15 fragments

\begin{tabular}{|c|c|c|c|c|}
\hline System & $\begin{array}{c}\text { Predicted Mass } \\
\text { Monomer / Dimer kDa }\end{array}$ & $\begin{array}{c}\text { SEC-MALS } \\
\mathrm{kDa}^{\dagger}\end{array}$ & $\begin{array}{l}\text { SEC-SAXS } \\
\mathrm{kDa}^{\dagger}\end{array}$ & State \\
\hline Hs PCDH15 EC2-3 WT $(n=3)$ & $29.3 / 58.7$ & $57.2(2.6 \%)$ & -- & Dimer \\
\hline Hs PCDH15 EC2-3 V250N $(n=3)$ & $29.3 / 58.7$ & $32.4(10.6 \%)$ & -- & Monomer \\
\hline Hs PCDH15 EC2-3 L306N/V307N $(n=3)$ & $29.3 / 58.7$ & $32.7(11.6 \%)$ & -- & Monomer \\
\hline Hs PCDH15 EC2-3 V250/L306N/V307N $(n=2)$ & $29.3 / 58.7$ & $30.1(2.7 \%)$ & -- & Monomer \\
\hline Hs PCDH15 EC1-3 WT $(n=1)$ & $43.0 / 86.0$ & $84.3(2.0 \%)$ & -- & Dimer \\
\hline Hs PCDH15 EC1-3 L306N/V307N $(n=1)$ & $43.0 / 86.0$ & $46.4(7.9 \%)$ & -- & Monomer \\
\hline Hs PCDH15 EC1-4 WT $(n=1)$ & $55.7 / 111.5$ & $106.7(4.3 \%)$ & -- & Dimer \\
\hline Hs PCDH15 EC1-4 L306N/V307N $(n=1)$ & $55.8 / 111.5$ & $57.9(3.8 \%)$ & -- & Monomer \\
\hline Mm PCDH15 EC9-MAD12 $(n=1)$ & $51.9 / 103.7$ & -- & $98.1(5.4 \%)$ & Dimer \\
\hline$M m^{*}$ PCDH15 EC1-3 WT $(n=1)$ & $43.7 / 87.4$ & $105.4(20.6 \%)$ & -- & Dimer \\
\hline$M m^{*}$ PCDH15 EC1-4 WT $(n=2)$ & $56.7 / 113.3$ & $139.6(21.9 \%)$ & -- & Dimer \\
\hline$M m^{*}$ PCDH15 EC1-4 V250N $(n=2)$ & $56.7 / 113.3$ & $72.6(26.7 \%)$ & -- & Monomer \\
\hline Mm* PCDH15 EC9-MAD12 WT $(n=1)$ & $52.9 / 105.7$ & $111.9(5.9 \%)$ & -- & Dimer \\
\hline$M m^{*}$ PCDH15 EC1-MAD12 WT $(n=1)$ & $152.4 / 304.8$ & $377.1(23.2 \%)$ & -- & Dimer \\
\hline Mm* PCDH15 EC1-MAD12 WT EDTA" $(n=1)$ & 152.4 / 304.8 & $355.8(16.3 \%)$ & -- & Dimer \\
\hline
\end{tabular}

+ Values in parenthesis indicate percentage deviation from predicted molecular mass.

* Mammalian expressed protein. The predicted mass represents that of the protein sequence without glycosylation. The calculated mass from SEC-MALS experiments is higher due to the presence of sugar moieties on the protein.

I EDTA was present in the SEC column but not directly added to the sample. 
bioRxiv preprint doi: https://doi.org/10.1101/695502; this version posted July 8 2019. The copyright holder for this preprint (which was not certified by peer review) is the author/funder, who has granted bioRxiv a license to display the preprint in perpetuity. It is made available under aCC-BY-NC-ND 4.0 International license.

Table 3. Overview of MD simulations

\begin{tabular}{|c|c|c|c|c|c|c|}
\hline System & Label & $\begin{array}{l}t_{\text {sim }} \\
\text { (ns) }\end{array}$ & Type & $\begin{array}{c}\text { Slowest speed } \\
\left(\mathrm{nm} \mathrm{ns}^{-1}\right)\end{array}$ & $\begin{array}{c}\text { Size } \\
\text { (\#atoms) }\end{array}$ & $\begin{array}{c}\text { Size } \\
\left(\mathrm{nm}^{3}\right)\end{array}$ \\
\hline $\begin{array}{l}\text { hs PCDH15 EC1-MAD12 + } \\
\text { CDH23 EC1-2 + } 3 \mathrm{Ca}^{2+} / \text { linker }\end{array}$ & S1a-d & 215.4 & $\mathrm{EQ} / \mathrm{SMD}$ & 0.1 & 924,024 & $71.2 \times 9.2 \times 14.9$ \\
\hline $\begin{array}{l}\text { mm PCDH15 EC1-MAD12 + } \\
\text { CDH23 EC1-3 + } 3 \mathrm{Ca}^{2+} / \text { /linker }\end{array}$ & $S 2 a-d$ & 296.2 & $\mathrm{EQ} / \mathrm{SMD}$ & 0.1 & $1,016,888$ & $91.7 \times 11.1 \times 10.5$ \\
\hline $\begin{array}{l}\text { hs (PCDH15 EC1-5 })_{2}+ \\
(\mathrm{CDH} 23 \mathrm{EC} 1-2)_{2}+3 \mathrm{Ca}^{2+} / \text { linker }\end{array}$ & S3a-d & 121.1 & $\mathrm{EQ} / \mathrm{SMD}$ & 0.1 & 833,064 & $40.5 \times 17.3 \times 12.4$ \\
\hline $\begin{array}{l}\text { hs (PCDH15 EC1-5) })_{2}+ \\
(\mathrm{CDH} 23 \mathrm{EC} 1-2)_{2}+2 \mathrm{Ca}^{2} / \text { linker }\end{array}$ & S4a-d & 125.1 & EQ/SMD & 0.1 & 833,037 & $40.5 \times 17.3 \times 12.4$ \\
\hline $\begin{array}{l}\text { hs (PCDH15 EC1-5) })_{2}^{+} \\
(\mathrm{CDH} 23 \mathrm{EC} 1-2)_{2}+1 \mathrm{Ca}^{2} / \text { linker }\end{array}$ & S5a-d & 157.2 & $\mathrm{EQ} / \mathrm{SMD}$ & 0.1 & 832,984 & $40.5 \times 17.3 \times 12.4$ \\
\hline $\begin{array}{l}\text { hs (PCDH15 EC1-5) })_{2}+ \\
(\mathrm{CDH} 23 \mathrm{EC} 1-2)_{2}+0 \mathrm{Ca}^{2} / \text { linker }\end{array}$ & S6a-d & 169.3 & EQ/SMD & 0.1 & 832,924 & $40.5 \times 17.3 \times 12.4$ \\
\hline $\begin{array}{l}m m(\mathrm{PCDH} 15 \mathrm{EC} 1-5)_{2}+ \\
(\mathrm{CDH} 23 \mathrm{EC} 1-3)_{2}+3 \mathrm{Ca}^{2+} / \text { linker }\end{array}$ & s7a-d & 114.1 & $\mathrm{EQ} / \mathrm{SMD}$ & 0.1 & 743,064 & $51.1 \times 11.7 \times 12.9$ \\
\hline $\begin{array}{l}\text { hs (PCDH15 EC1-MAD12) }+ \\
(\mathrm{CDH} 23 \mathrm{EC} 1-2)_{2}+3 \mathrm{Ca}^{2+} / \text { linker }\end{array}$ & S8a-d & 153.2 & $\mathrm{EQ} / \mathrm{SMD}$ & 0.1 & $1,178,985$ & $99.4 \times 11.4 \times 10.9$ \\
\hline $\begin{array}{l}m m(\mathrm{PCDH} 15 \mathrm{EC} 1-\mathrm{MAD} 12)_{2}+ \\
(\mathrm{CDH} 23 \mathrm{EC} 1-3)_{2}+3 \mathrm{Ca}^{2+} / \text { linker }\end{array}$ & S9a-d & 170.6 & $\mathrm{EQ} / \mathrm{SMD}$ & 0.1 & $1,292,017$ & $91.5 \times 11.8 \times 12.5$ \\
\hline $\begin{array}{l}m m(\mathrm{PCDH} 15 \mathrm{EC} 1-\mathrm{MAD} 12)_{2}+ \\
(\mathrm{CDH} 23 \mathrm{EC} 1-3)_{2}+3 \mathrm{Ca}^{2+} / \text { linker }\end{array}$ & S10a & 95.3 & EQ & - & $2,325,962$ & $65.5 \times 18.9 \times 19.5$ \\
\hline $\begin{array}{l}m m(\mathrm{PCDH} 15 \mathrm{EC9}-\mathrm{MAD} 12)_{2}+3 \\
\mathrm{Ca}^{2+} / \text { linker }\end{array}$ & S11a-d & 124.8 & $\mathrm{EQ} / \mathrm{SMD}$ & 0.1 & 456,282 & $28.2 \times 13.2 \times 13.2$ \\
\hline
\end{tabular}

